University of Louisville

ThinkIR: The University of Louisville's Institutional Repository

$5-2020$

\title{
Transient display of chimeric proteins on biological surfaces as an effective strategy for modulations of innate and adaptive immune responses.
}

Pradeep Shrestha

University of Louisville

Follow this and additional works at: https://ir.library.louisville.edu/etd

Part of the Medical Immunology Commons

\section{Recommended Citation}

Shrestha, Pradeep, "Transient display of chimeric proteins on biological surfaces as an effective strategy for modulations of innate and adaptive immune responses." (2020). Electronic Theses and Dissertations. Paper 3413.

https://doi.org/10.18297/etd/3413

This Doctoral Dissertation is brought to you for free and open access by ThinkIR: The University of Louisville's Institutional Repository. It has been accepted for inclusion in Electronic Theses and Dissertations by an authorized administrator of ThinkIR: The University of Louisville's Institutional Repository. This title appears here courtesy of the author, who has retained all other copyrights. For more information, please contact thinkir@louisville.edu. 
TRANSIENT DISPLAY OF CHIMERIC PROTEINS ON BIOLOGICAL SURFACES AS AN EFFECTIVE STRATEGY FOR MODULATION OF INNATE AND ADAPTIVE IMMUNE RESPONSES

\author{
By \\ Pradeep Shrestha \\ B.Sc, Tribhuvan University \\ M.Sc., Tribhuvan University \\ M.S., University of Louisville
}

A Dissertation Submitted to the Faculty of

the School of Medicine at the University of Louisville

in Partial Fulfillment of the Requirements

for the Degree of

Doctor of Philosophy in Microbiology and Immunology

Department of Microbiology and Immunology

University of Louisville

Louisville, Kentucky

May 2020 


\section{Copyright 2020 Pradeep Shrestha}

All rights reserved 

TRANSIENT DISPLAY OF CHIMERIC PROTEINS ON BIOLOGICAL SURFACES AS AN EFFECTIVE STRATEGY FOR MODULATION OF INNATE AND ADAPTIVE IMMUNE RESPONSES

\author{
By \\ Pradeep Shrestha \\ B.Sc, Tribhuvan University \\ M.Sc., Tribhuvan University \\ M.S., University of Louisville
}

April 8, 2020

by the following Dissertation Committee:

Dissertation Director: Haval Shirwan, Ph.D.

Dissertation Co-Director: Esma Yolcu, Ph.D.

Committee Member: Nejat K Egilmez, Ph.D.

Committee Member: Mariusz Ratajczak, M.D., Ph.D., D.Sci

Committee Member: Bing Li., Ph.D 


\section{DEDICATION}

To my parents who supported and inspired me to work hard to pursue my career

To my brother who adores and tells me to have patience and hope

To my dear wife who has been enduring me, caring for me and supporting me mentally, emotionally and physically and makes me a better person. 


\section{ACKNOWLEDGEMENT}

Foremost, I would like to express my sincere gratitude to my mentor Dr. Haval Shirwan, for the opportunity and continuous support for my $\mathrm{PhD}$ research. His continuous motivation, guidance, enthusiasm and support made my research and dissertation possible. I am also truly grateful to my co-mentor Dr. Esma S. Yolcu for believing in me and providing the opportunity to work with her. Her guidance for quality research, motivation for hard work and exceptional support has definitely made this journey a success. I am very much thankful to my committee members Dr. Nejat K. Egilmez, Dr. Mariusz Ratajczak and Dr. Bing Li for their time, support and scientific insights that helped to progress this study. I am also truly grateful to all my past and present lab members Hong Zhao, Kyle Woodward, Hampartsoum Barsoumian, Helen Tan, Feng Zhang, Orlando Grimany, Ali Turan, Alper Togay, Christine Akimana, Lalit Batra, Tariq Malik, Lei Zhang and others for their help, trainings and friendship that made this journey pleasant and easy. I also would like to thank our collaborators: Dr. Andreas Garcia and Dr. Maria Coronel at Georgia Tech and Drs. Subha R. Das, Phil Campbell, Sai Yerneni and others at Carnegie Mellon University for the opportunity to work with these brilliant minds. I am also thankful to Melissa for helping me with blood withdraw. Special thanks to other helping hands, Dr. Rajdeep Bomjan and my parents, my brother and my dear wife for their continuous support and encouragement to pursue my goals and career aspiration. Finally, I would like to thank the department of Microbiology and Immunology, the Institute for Cellular therapeutics for providing great working environment. 


\begin{abstract}
TRANSIENT DISPLAY OF CHIMERIC PROTEINS ON BIOLOGICAL SURFACES AS AN EFFECTIVE STRATEGY FOR MODULATION OF INNATE AND ADAPTIVE IMMUNE RESPONSES

Pradeep Shrestha
\end{abstract}

April 8, 2020

The major premise of this dissertation was to transiently display novel immunological ligands on biological membranes as a localized means of modulating innate and adaptive immune responses with applications to bone marrow and pancreatic islet transplantation. In Chapter two, we engineered donor allogeneic bone marrow cells to transiently display a novel form of FasL, SA-FasL, to efficiently purge out alloreactive donor T cells to prevent acute GVHD. In Chapter three, we engineered pancreatic islets with a novel form of CD47, SA-CD47, to modulate instant blood mediated inflammatory reaction (IBMIR) to prevent immediate islet graft loss following intraportal transplantation.

GVHD is initiated and perpetuated by mature T cells in the bone marrow inoculum following transplantation into conditioned recipients. Upon activation, T cells upregulate Fas receptor and become sensitive to FasL-mediated apoptosis. Thus, we hypothesized that the display of SA-FasL on $\mathrm{T}$ cells in bone marrow will result in their apoptosis potentially in autocrine fashion following activation in response to recipient alloantigens and engagement of Fas with SA-FasL on the T cells, thereby resulting in the prevention of 
acute GVHD. We demonstrated that SA-FasL engineered T cells underwent apoptosis following response to alloantigens both in vitro and in vivo. Most importantly, in an haploidentical rodent setting where C57BL/6 bone marrow cells containing $\mathrm{T}$ cells transplanted into lethally irradiated F1 recipients, engineering cells with SA-FasL resulted in the prevention of lethal acute GVHD in $80 \%$ of recipients long term (>100 days). We extended this observation to xenogeneic acute GVHD setting, where mice receiving SAFasL-engineered human PBMCs were significantly protected.

Significant islet mass loss following intraportal transplantation is a major barrier for clinical islet transplantation. IBMIR is initiated and perpetuated by innate immune cells. $\mathrm{CD} 47-\mathrm{SIRP} \alpha$ axis known as innate immune checkpoint delivers "don't eat me signal" to prevent phagocytosis and activation of myeloid cells. Thus, we hypothesized that engineering islets to transiently display SA-CD47 as an innate immune checkpoint will mitigate IBMIR and enhance engraftment following intraportal transplantation. In a syngeneic marginal mass model of intraportal transplantation, SA-CD47-islets showed better engraftment and function as compared with the control group (87.5 vs 14.3\%). Engraftment was associated with low levels of intrahepatic inflammatory cells and mediators of islet destruction, including HMBG-1, tissue factor, and IL-1 $\beta$. Overall, we show that transient display of immunological ligands on biological membranes is effective in modulating innate and adaptive immune responses with significant translational implication for multiple immune-based disorders. 


\section{TABLE OF CONTENTS}

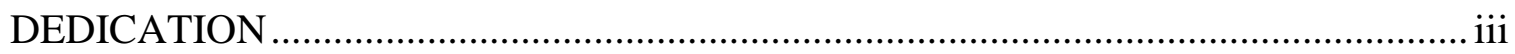

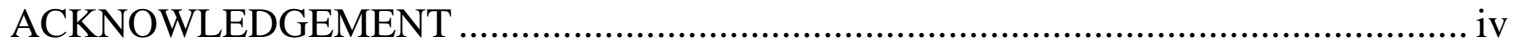

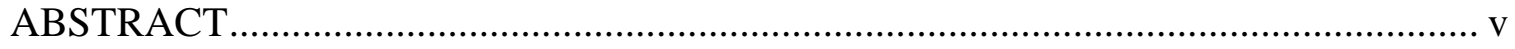

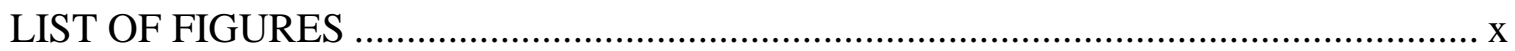

LIST OF TABLES ....................................................................................... xiii

CHAPTER 1: INTRODUCTION ........................................................................ 1

Overview on hematopoietic stem cell transplantation (HSCT) .............................. 1

Immunobiology of acute graft-versus-host disease ....................................... 2

Three-phase model of acute GVHD ....................................................... 3

Preventive and therapeutic strategies against acute GVHD .......................... 8

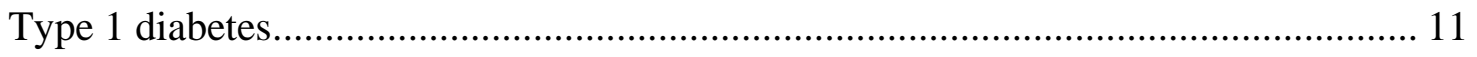

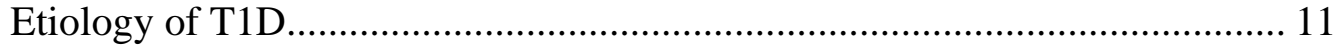

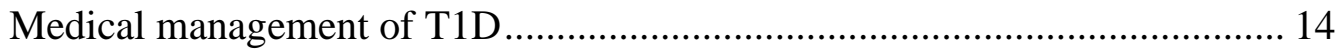

Pancreatic islet transplantation ........................................................ 15

Instant blood mediated inflammatory reaction (IBMIR) .................................... 18

Strategies to prevent IBMIR ................................................................ 19

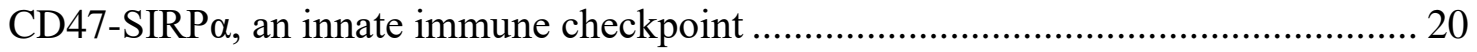


ProtEx ${ }^{\mathrm{TM}}$ technology as a facile and effective platform for localized immunomodulation

\section{CHAPTER 2 TARGETING FAS-FASL PATHWAY FOR SELECTIVE \\ ELIMINATION OF ALLOREACTIVE DONOR CELLS FOR PREVENTION OF}

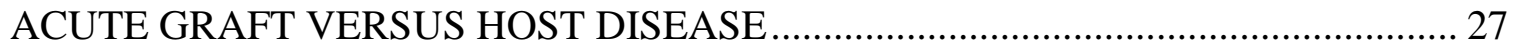

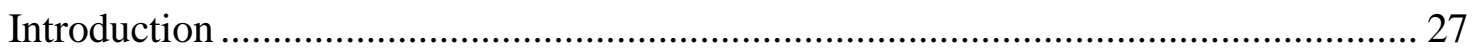

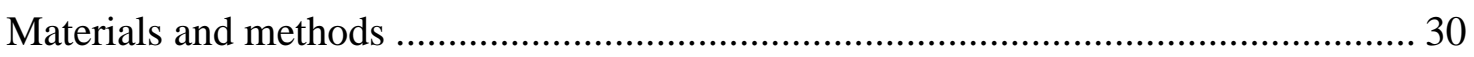

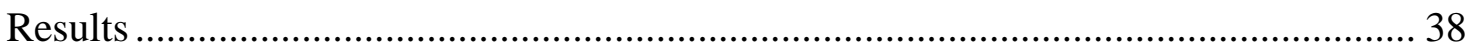

$\mathrm{T}$ effector cells engineered with SA-FasL are efficiently eliminated in response

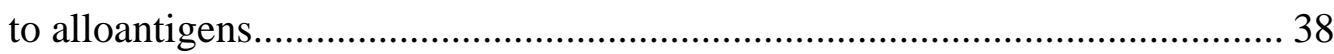

Transient display of SA-FasL on donor T cells Engineering of donor graft to display SA-FasL efficiently prevents lethal acute GVHD ................................ 46

Long term SA-FasL-engineered donor cell recipients are functionally immune

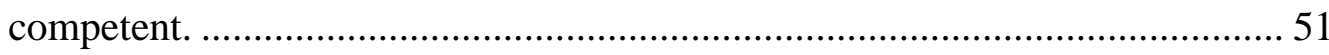

SA-FasL recipients exhibit reduced activated alloreactive Th1 phenotype..... 53 Donor $\mathrm{CD} 25^{+}$Treg cells are indispensable for the prevention of acute GVHD.

SA-FasL engineering of human PBMCs abrogates acute GVHD in a

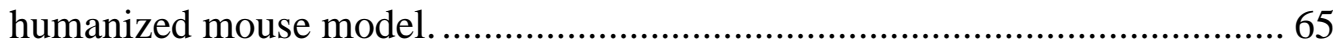

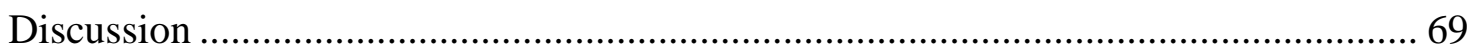




\section{CHAPTER 3 PANCREATIC ISLETS SURFACE ENGINEERED WITH CD47}

INNATE IMMUNE CHECKPOINT SHOW ENHANCED ENGRAFTMENT

FOLLOWING INTRAPORTAL ISLET TRANSPLANTATION ............................. 75

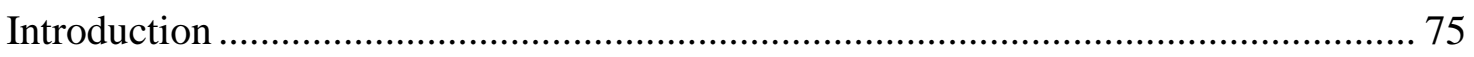

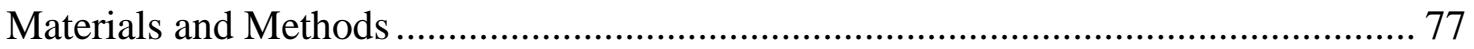

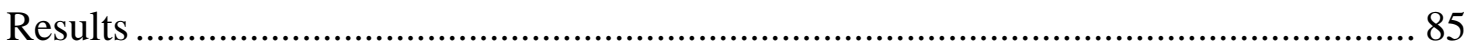

Generation and characterization of chimeric SA-CD47 protein ................... 85

Effective engineering of islets with SA-CD47 protein without negatively

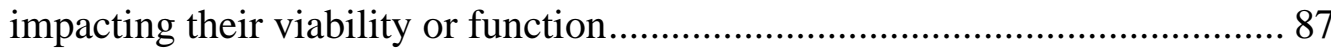

SA-CD47-engineered xenogeneic cells are refractory to phagocytosis by

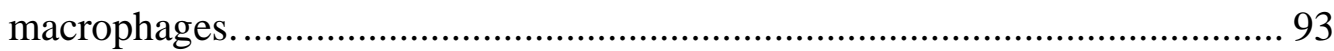

SA-CD47 protects islets and prevents intra-islet infiltration of CD11 $\mathrm{b}^{+}$cells in an in vitro loop assay.

SA-CD47-engineered islets show enhanced engraftment and function following intraportal transplantation

SA-CD47 display alters the intrahepatic infiltration of inflammatory cells and inflammatory mediators. 103

Discussion 107

CHAPTER 4: SUMMARY, IMPLICATION AND FUTURE DIRECTION 111

REFERENCES 114

CURRICULUM VITAE 142 


\section{LIST OF FIGURES}

FIGURE

PAGE NUMBER

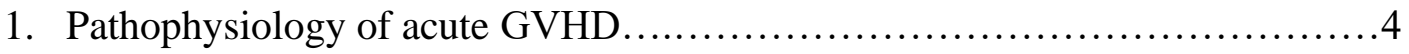

2. Progression of Type-1 diabetes............................................

3. Pancreatic islet transplant procedure and factors contributing to

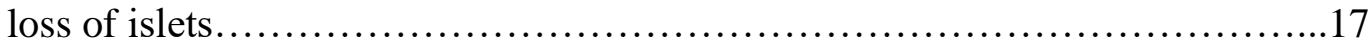

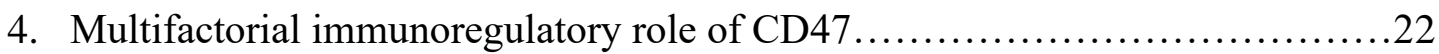

5. ProtEx ${ }^{\mathrm{TM}}$ technology to display multiple immunomodulatory proteins on the

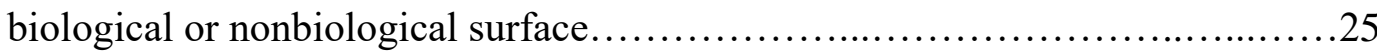

6. SA-FasL transiently displayed on the surface of $\mathrm{T}$ cells is effective in blocking their proliferation in response to alloantigens in vitro ...........................39

7. Elimination of alloreactive cells by SA-FasL engineering in an allogeneic adoptive transfer model

8. SA-FasL engineered alloreactive cells are eliminated in an in vivo model.

9. Engineering donor graft with SA-FasL abrogates lethal acute GVHD and show efficient lymphoid reconstitution.........................................48

10. Long term SA-FasL-spleen recipients show full donor chimerism...............49

11. Immunophenotyping analysis of long-term animals..........................50

12. Long term SA-FasL-spleen recipients are immune competent..................52 
13. SA-FasL-spleen recipients have less activated cells and inflammatory mediators of acute GVHD at day 21 post transplantation.......................56

14. Immunophenotyping of recipients at day 21 post transplantation................58

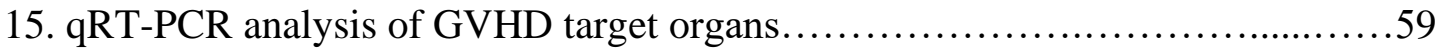

16. Intracellular cytokine analysis on spleen cells..............................60

17. Representative H\&E staining of GVHD target organs from each cohort.........61

18. Depletion of $\mathrm{CD} 25^{+}$cells from donor inoculum...........................63

19. CD25+ cells are indispensable for observed protective effect of SA-FasL for acute

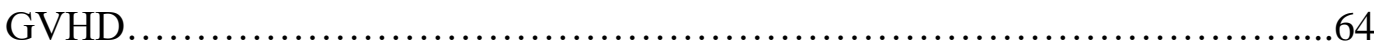

20. Frequency of human immune cells recovered from preconditioned NSG

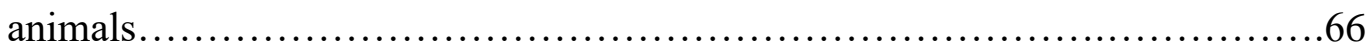

21. SA-FasL engineering eliminates activated human PBMCs and abrogates

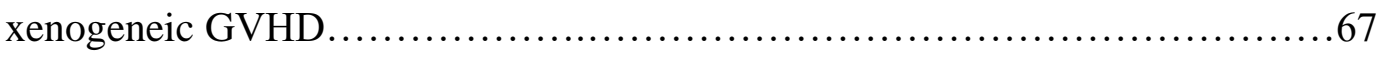

22. Cloning, expression, and structural characterization of SA-CD47 protein.........86

23. Representative histogram overlay plot of spleen cells engineered

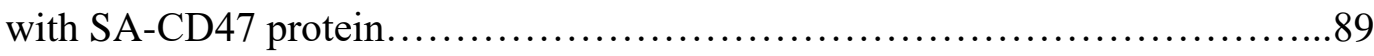

24. Effective engineering of islets with SA-CD47 protein without a negative impact on their viability or function.............................. 90

25. Bright field images showing integrity of SA-CD47-engineered islets...........92

26. SA-CD47 protein on xenogeneic cells prevents phagocytosis by

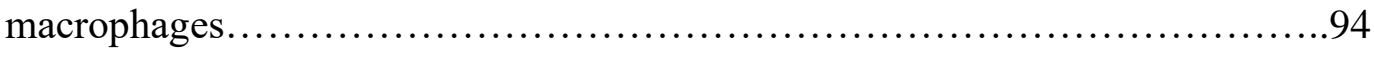

27. Representative flow plots of in vitro phagocytosis assay .......................95

28. SA-CD47 engineering protects islets from destruction by IBMIR 
in an in vitro loop assay.

29. Representative confocal images for CD11b negative staining....................98

30. Surface display of SA-CD47 protein significantly improves pancreatic islet engraftment and function in a syngeneic marginal mass intraportal transplantation model.

31. SA-CD47 engineering improves the intraportal islet transplantation outcome. 101

32. SA-CD47 engineered islet grafts show long term survival and function following intraportal transplantation. 102

33. SA-CD47-islet recipients have significantly reduced infiltration of inflammatory cells and mediators in liver following intraportal transplantation. .105

34. SA-CD47-islet graft recipients have substantially less intra-hepatic infiltration of inflammatory cells 106 


\section{LIST OF TABLES}

Table

Page number

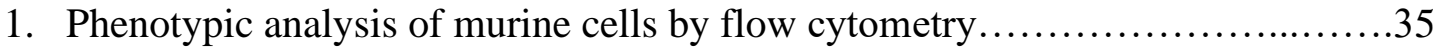

2. Phenotypic analysis of human cells by flow cytometry...........................

3. TaqMan assay for indicated gene expression in GVHD tissues................37

4. TaqMan gene expression assay for islet graft analysis......................... 83 


\section{CHAPTER 1: INTRODUCTION}

\section{Overview on hematopoietic stem cell transplantation (HSCT)}

Hematopoietic stem cells or bone marrow transplantation is a well-established treatment care for hematologic and non-hematologic malignancies that are not curable by conventional treatment procedures ${ }^{1,2}$. Over the last 50 years, HSCT procedure has evolved from a highly experimental technique to an effective cellular immunotherapeutic treatment against a number of malignancies, metabolic deficiencies, and autoimmune diseases ${ }^{3,4}$. With advances in patient-donor selection, reduced toxicity conditioning regimen, stem cell sources and supportive care, HSCT procedure has seen a significant progress. Annually an estimated 50,000 procedures are done worldwide and more than 1 million transplantations have been performed with $40 \%$ being allogeneic in nature ${ }^{2,5}$.

The HSCT may be autologous or allogeneic in nature. Autologous transplantation procedure involves infusion of patient's own stem cells. The procedure is mainly performed as a strategy to rescue from lethal chemotherapy against underlying malignancy. Allogeneic transplantation, where immunologically disparate hematopoietic stem cells and immunologic repertoire are infused into irradiation or chemotherapy conditioned patient to establish donor derived hematopoiesis and immunity ${ }^{6}$. Allogeneic transplant modality is currently extended to maintain mixed donor chimerism to establish solid organ graft tolerance $^{7-9}$. Despite the advances, allogeneic HSCT is associated with a major life- 
threatening complication, graft-versus-host disease (GVHD), thus limiting the use of this important procedure. GVHD occurs when the immunocompetent donor cells in the graft recognizes the recipient as foreign. The resulting immune response activates donor $\mathrm{T}$ cells to attack and eliminate recipient cells as foreign antigen bearing cells ${ }^{2}$.

There are two main clinical presentation of GVHD: acute and chronic GVHD with different etiologies and pathophysiology. Acute GVHD was initially defined as a condition appearing within 100 days post transplantation whereas chronic GVHD being later ${ }^{1,10}$. However, this distinction is not tenable anymore as acute GVHD may present beyond 3 months, whereas chronic GVHD may contract within 100 days post transplantation. The present diagnosis criteria involve the specificity of sign and symptoms, rather than the time

of onset $^{11,12}$. Acute GVHD manifests as infiltration of inflammatory $\mathrm{T}$ cells with target tissue destruction particularly skin, gastrointestinal tract, and liver. On the other hand, chronic GVHD involves the Th2 immune response, autoimmune disease characteristics including autoantibody formation in skin and mucosal surfaces. Acute GVHD is primarily driven by activated $\mathrm{T}$ cells and pro-inflammatory cytokines, whereas chronic GVHD is more complex and involving the interaction of innate immune cells with dysregulated B and $\mathrm{T}$ cells ${ }^{2,13}$.

\section{Immunobiology of acute graft-versus-host disease}

Acute GVHD is a severe inflammatory complication of allogeneic HSCT. Despite the advance in preventive and post-transplant strategies, acute GVHD is still considered a significant cause of morbidity and mortality in allogeneic HSCT recipients. Billingham proposed three distinct requirements for GVHD reaction: i) immune responsive donor cells 
in the graft; ii) Antigenic disparity between donor and recipient; iii) immune suppressed recipient system that cannot eliminate transplanted donor cells ${ }^{12,14}$. It is now well established that immune competent cells are donor T cells that primarily drive GVHD and can occur in different clinical settings when tissue (bone marrow, blood products or solid organ) containing $\mathrm{T}$ cells are transplanted to recipient that is unable to eliminate those cells $^{2,12,15}$.

\section{Three-phase model of acute GVHD}

The progression of acute GVHD can be summarized in a three step process with an afferent and efferent phase. Phase 1 involves the effect of conditioning in which irradiation and chemotherapy as conditioning regimen induces injury to host epithelium and endothelium generating proinflammatory cytokines and recruits innate immune cells. Phase 2 involves the process of allorecognition, activation and proliferation of donor $\mathrm{T}$ cells in the inflammatory milieu induced in phase 1 . These two phases make afferent phase of acute GVHD. Whereas in phase 3 as efferent phase the activated multiple effector cells, cytotoxic T cells (CTLs), natural killer (NK) cells and large granular lymphocytes cause significant damage and further injury in specific and non-specific ways ${ }^{12,16,17}$. The threephase model of acute GVHD is summarized in figure 1.

\section{Phase 1: Effects of conditioning}

HSCT recipient undergoes conditioning regimens before transfusion of donor grafts. The procedure involves irradiation and/or chemotherapy. The conditioning therapy is rather toxic to recipient tissues and induces tissue damage. Underlying disease and conditioning induce tissue injury and respond by releasing proinflammatory cytokines (TNF- $\alpha$, IL-1 and IL6). 


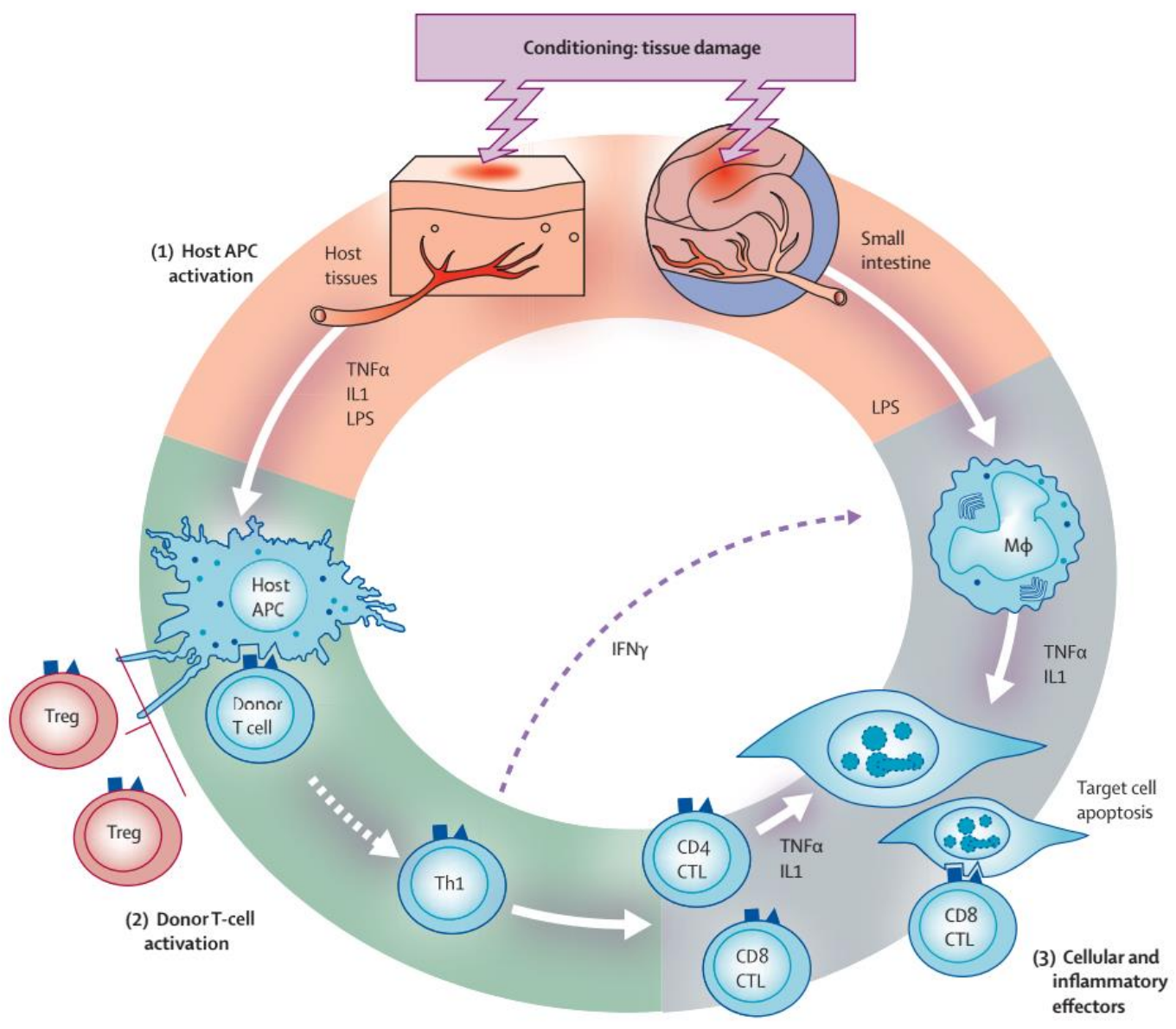

Figure 1: Pathophysiology of acute GVHD. The three phases of acute GVHD. 1. Activation of host APCs because of tissue damage due to conditioning regimen. 2 . Activation, differentiation and proliferation of donor T cells by host APCs. 3. The effector phase where complex cascade of effector cells and inflammatory mediators in presence of chemokines and cytokines mediate host tissue damage. (Adapted from Ferrara et. al. Lancet 2009). 
The proinflammatory cytokines upregulates the expression of chemokines and other adhesion molecules, MHC antigens, costimulatory molecules in antigen presenting cells $(\mathrm{APCs})^{2,12,18,19}$. In addition, gastrointestinal tract is particularly susceptible to injury during the conditioning process. This leads to systemic infusion of inflammatory stimuli such as lipopolysaccharide or other pathogen associated molecular patterns that stimulates gut associated lymphocytes and macrophages to produce TNF- $\alpha$ and IL-1. This directly contributes to "cytokine storm"2. Alongside, danger signals such as ATP, uric acid, high mobility group box-1, heparan sulfate released during tissue damage can activate inflammasomes ${ }^{2,20}$. This leads to cleavage of pro-IL- $1 \beta$ to its bioactive form IL- $1 \beta$ and thus enhancing GVHD. This surge of cytokines along with danger signals is important for initiation of primary and secondary immune response. Direct correlation between intensive conditioning regimen and acute GVHD severity is well established and clinical studies suggests that reduced intensity conditioning is associated with significantly reduced morbidity and less early acute GVHD ${ }^{17,21}$.

\section{Phase 2: Activation of donor $\mathrm{T}$ cells}

Interaction between donor T cells and recipient APCs play central role in acute GVHD. This interaction leads to their subsequent activation, proliferation and differentiation and is the second afferent stage in progression of acute GVHD. Donor T cells can be activated directly by host derived or indirectly by donor derived APCs. Presentation of alloantigens directly by host APCs appear to be critical in inducing acute GVHD although indirect presentation by recipient APCs also plays role in activation of donor $\mathrm{T}$ cells ${ }^{22,23}$. Dendritic cells (DCs) are the potent APCs during the process. 
Inflammatory cytokines (TNF- $\alpha$, IL-1), danger associated molecular patterns (DAMPs) and pathogen associated molecular pattern (PAMPs) produced during phase 1 (conditioning effects) play important roles in maturation of DCs and induce activation of donor T cells whereas immature DCs induce T cell tolerance ${ }^{2,12,24}$.

In addition to engagement of suitable TCR to alloantigen presenting MHC molecule, second costimulatory signal is required for full activation of $\mathrm{T}$ cells. Costimulatory signals lower the $\mathrm{T}$ cell activation threshold, inhibit apoptosis signaling, maintain cytokine production and support the metabolism of effector T cells. Multiple costimulatory pathways including ICOS, CD28, OX40, and $41 \mathrm{BB}^{2,25,26}$ and negative regulatory pathway including CTLA-4, PD1-PDL1, have been shown to regulate progression of acute GVHD ${ }^{26,27}$.

Finally, cytokines play important role in maintenance of $\mathrm{T}$ cell activation and survival that drives acute GVHD. Multiple cytokines including IL-1 $\beta$ and Th1 cell cytokines (IFN- $\gamma$, IL-2 and TNF- $\alpha$ ) mediate $\mathrm{T}$ cells differentiation and GVHD pathogenesis $^{12}$. For instance, IFN- $\gamma$ plays a crucial role in pathophysiology of acute GVHD. T cells isolated in experimental and clinical acute GVHD produce large amounts of IFN$\gamma^{28}$. IFN- $\gamma$ upregulates chemokine expression, MHC molecule and adhesion molecules such that it facilitates antigen presentation and effector recruitment ${ }^{17}$. Also, IFN- $\gamma$ plays important role in regulating apoptotic death of activated $\mathrm{T}$ cells by regulating Fas receptor, thus regulating GVHD ${ }^{29-31}$. In addition, IFN- $\gamma$ exposure significantly reduces the threshold of LPS required to production of proinflammatory cytokines and Nitric oxide (NO) by macrophages ${ }^{17,32}$. 


\section{Phase 3: Cellular and Inflammatory effector phase}

Multiple cellular and inflammatory mediators mediate host tissue damage during the effector/efferent phase. It is a complex cascade where inflammatory agents (eg. NO. TNF- $\alpha$, IL-1) and cellular mediators (eg. Cytotoxic T cells, NK cells) work in synergy to amplify host tissue damage thus promoting inflammation and target tissue injury. The primary cellular effectors are CTLs and NK cells that mediate direct tissue damage ${ }^{33}$. They can mediate cytotoxicity by two main pathways. Contact dependent ligation of Fas-FasL results in activation of death-inducing signaling complex (DISC). Activation of DISC results to activation of caspases ultimately leading to apoptotic cell death ${ }^{34-36}$. During GVHD, hepatocytes, epithelial cells on bile ducts upregulate expression of Fas receptor making them susceptible to FasL mediated cytotoxic effect. In contrast, gastrointestinal damage is preferentially mediated by perforin-granzyme-B cytotoxic pathway ${ }^{37,38}$.

Activated mononuclear phagocytes are major source of inflammatory mediators including TNF- $\alpha$, IL-1 that promote direct tissue damage in acute GVHD. TNF- $\alpha$ plays a central role in pathogenesis of acute GVHD. It plays role in activation of DCs and promotes antigen presentation. Also, regulates the recruitment of effector cells (neutrophils, monocytes, and effector T cells) to target organ by induction of inflammatory chemokines $\left(\right.$ CCL2-CCL5, CXCL2, CCL17) ${ }^{12,17}$. In addition, TNF- $\alpha$ can induce apoptosis and necrosis directly on tissues ${ }^{33,39}$. Nitric oxide (NO) is another inflammatory mediator in acute GVHD. NO promotes pathophysiology of acute GVHD by inducing immunosuppression an inhibiting repair mechanism of target tissue. This results in inhibition of proliferation of epithelial stem cells in gut and skin and induction of direct tissue damage $\mathrm{e}^{32,40,41}$. 
Thus, the complex synergistic interaction between cytotoxic effector cells and inflammatory mediators in presence of chemokines and cytokines results in amplification of local tissue injury thus further promoting inflammatory response that ultimately results in observed tissue destruction in the transplant recipient.

\section{Preventive and therapeutic strategies against acute GVHD}

Cyclosporine-A, a calcineurin inhibitor and a cytotoxic agent methotrexate (MTX) or mycophenylate mofetil (MTF) are commonly used as standard preventive pharmacologic agents against acute GVHD by multiple clinical centers. MTX/MTF and cyclosporine exert their antiproliferative effect on activated donor $\mathrm{T}$ cells by interfering with purine synthesis and calcium dependent TCR signaling respectively. With discovery of tacrolimus, another calcineurin inhibitor with similar mechanism to cyclosporine-A, that has similar or superior efficacy to cyclosporine is now widely used in clinical HSCT ${ }^{2,12,15}$. Tacrolimus/ cyclosporine exert their effect by inhibiting the calcineurin that is required for the activation of nuclear factor of activated T cell (NFAT) family transcription factor that is required for transcription of IL-2 and activation of $\mathrm{T}$ cells ${ }^{42}$. Sirolimus, an mTOR inhibitor, is more potent inhibitor of activation and proliferation of conventional $\mathrm{T}$ cells than regulatory $\mathrm{T}$ cells (Treg cells) owing to dependence of conventional T cells on mTORkinase B. High dose of cyclophosphamide is currently used in haploidentical HSCT settings. Two doses of cyclophosphamide are infused immediately post transplantation to eliminate highly proliferating alloreactive $\mathrm{T}$ cells but concomitantly sparing of stem cells and Treg cells. Expression of high level of aldehyde dehydrogenase in Treg cells and stem cells is a major in vivo mechanism to cyclophosphamide resistance ${ }^{43}$. 
Besides the use of pharmacologic agents, manipulation of donor $\mathrm{T}$ cells as a prophylactic strategy has been employed in multiple experimental and clinical HSCT settings. One such strategy employs $\mathrm{T}$ cell depleted bone marrow graft transplant followed by delayed donor lymphocyte $\mathrm{T}$ cell infusion ${ }^{44-46}$. Delayed $\mathrm{T}$ cell add back strategy circumvented acute GVHD and restored graft-versus-leukemia (GVL) effect; however, the antileukemic effect was not as efficient as $\mathrm{T}$ cell replete transplants ${ }^{44}$. Another strategy involves $\mathrm{T}$ cell depletion from the graft. These methods include ex vivo negative selection (e.g. monoclonal antibody, lectin agglutination), ex vivo positive selection of $\mathrm{CD}^{2} 4^{+}$cells, or in vivo depletion (anti $\mathrm{T}$ cell antibody preparation). However, these strategies substantially limited acute GVHD incidences but did not translate to improved overall survival because of graft failure, Epstein-Bar-Virus associated lymphoproliferative disorder, and disease relapse $e^{2,45,47}$. A more subtle strategy to define anti-host GVHD causing $\mathrm{T}$ cells and their depletion have been reported. This strategy targets activation induced antigens; whose expression is upregulated on alloreactive T cells upon stimulation in allogeneic mixed lymphocyte reaction culture. Selective depletion of activated cells based on activation markers (such as CD25, CD69, HLA-DR, CD134 and CD137) using fluorescence activated cell sorting (FACS) or CFSE dilution ${ }^{48}$ or CD25 conjugated immunotoxin ${ }^{49}$ have been reported to improve the outcome of $\mathrm{HSCT}^{47,48,50,51}$. These specific alloreactive $\mathrm{T}$ cell depletion approach significantly reduces GVHD without losing $\mathrm{T}$ cell associated graft-supporting properties and early immune reconstitution ${ }^{50,52}$.

Multiple cellular therapy approaches have been tested. Adoptive transfer of ex vivo expanded Treg cells along with $\mathrm{T}$ cell replete graft was associated with significantly reduced incidence of acute GVHD with better survival ${ }^{53}$. In addition, adoptive transfer of 
donor lymphocytes co-cultured with IL-10 treated host APCs in presence of TGF- $\beta$ to enrich IL-10 producing type 1 regulatory $T$ (Tr1) cells showed enhanced immune reconstitution with reduced acute $\mathrm{GVHD}^{2}$.

Despite the prophylaxis, acute GVHD still evolves and the first line of therapy is systemic glucocorticoids like prednisone that have potent anti-lymphocyte and antiinflammatory activity. Patients refractory to steroid treatment have dismal long term prognosis with overall survival rate of only $5-30 \%{ }^{2}$. More importantly, acute GVHD restrict the HSCT to haploidentical population. Thus, there is an acute need for the development of less toxic and more efficacious approaches for bone marrow transplantation that goes beyond haploidentical. 


\section{Type 1 diabetes}

Diabetes mellitus is a chronic metabolic disorder characterized by persistently increased blood glucose level (BGL). The higher BGL in an individual being defined as fasting glucose level $\geq 125 \mathrm{mg} / \mathrm{dl}$ or random non-fasting glucose level $\geq 200 \mathrm{mg} / \mathrm{dl}$ or hemoglobin Alc $(\mathrm{HbAlc}) \geq 6.5 \%{ }^{54,55}$. Persistent higher BGL can cause ketoacidosis, vascular injury, kidney failure, heart disease, stroke and blindness. The world prevalence of diabetes in adults was $6.4 \%$ in 2010 and expected to rise to $7.7 \%$ by $2030^{55}$. Every year diabetes is associated with 200,000 deaths and costs $\$ 245$ billion in US alone ${ }^{56}$.

Type 1 diabetes (T1D) is a classical $\mathrm{T}$ cell mediated autoimmune disorder associated with loss of pancreatic $\beta$-cells. Significant loss of $\beta$-cells leads to insulin deficiency resulting in hyperglycemia and ketoacidosis ${ }^{54}$. Although it is termed as "juvenile diabetes" due to more frequent diagnosis in children, majority of patients with T1D are adults and represents $10-15 \%$ of total diabetes mellitus cases worldwide. Annually, in average 78,000 youths are diagnosed with T1D worldwide and in US alone > 20 cases per 100,000 people and the incidence rate is expected to rise $\mathrm{e}^{56-58}$.

\section{Etiology of T1D}

T1D is a polygenic heritable disease and at least 20 different chromosomal regions are associated to its susceptibility. For instance, identical twin concordance rate of T1D is $30-70 \%$, sibling risk of 3-7\% and risk of 1-9\% for children with parents who have T1D ${ }^{59,60}$. Multiple genetic factors contribute to both susceptibility as well as resistance to precipitation of T1D. One of the largest contributions comes from several genes located in MHC complex on chromosome 6p21 (i.e IDDM1 locus). Two HLA class II haplotypes $H L A-D R 3 / 4-D Q 2 / 8$, involved in antigen presentation, are associated with high risk or 


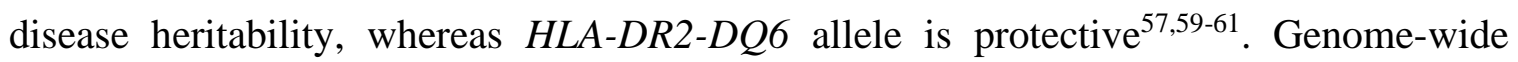
association studies have identified multiple non-HLA alleles, VNTR, PTPN22, CTLA4, $I L 2 R A$, associated with susceptibility to T1D. These genetic variants are involved in immune response that contributes to dysfunctional immune responsiveness, including development and maintenance of tolerance ${ }^{60}$. Despite the genetic factors, the concordance rate of T1D among monozygotic twins is only 50\%, implicating the potential role of environmental factors in development and precipitation of T1D. One of the major candidates is viral infection. Extensive data suggests coxsackieviruses, an enterovirus infection precipitates $\mathrm{T}_{1} \mathrm{D}^{62}$. With evidence of enterovirus in pancreas of recent onset $\mathrm{T} 1 \mathrm{D}$ patients and epidemiological studies suggesting significantly more enterovirus infection among diabetic patients than in controls supports the notion that viral infections are the environmental triggers. Many viruses can cause chronic or latent infection. Direct evidence comes from a study where coxsackievirus can infect $\beta$-cells and cause insulitis and diabetes in murine models ${ }^{63}$. With the landmark finding by Foulis et al. ${ }^{64}$ it is suggested that $\beta$-cell tropic viral infection upregulates HLA class I and IFN- $\alpha$ as "viral molecular signatures". In addition, significant sequence similarity between coxsackievirus protein $2 \mathrm{C}$ and glutamic acid decarboxylase (GAD) protein, a major T1D autoantigen, postulates the viral mimicry as an etiology of $\mathrm{T}^{1} \mathrm{D}^{65}$. Coxsackievirus induced upregulation of CXCL10 chemokine on pancreatic islets plays important role in recruitment of CXCR3 positive autoreactive $\mathrm{T}$ cells following viral infection ${ }^{66}$. Cumulatively, available data suggest the significant role of environmental triggers, viral infections, microbiome composition and metabolites, milk and wheat proteins, in unleashing autoimmunity, leading to destruction of beta cells that ultimately results in T1D. 
Silent immune events targeting the destruction of pancreatic $\beta$-cells may take years before clinical symptoms of T1D becomes apparent. Autoantibodies and activated autoreactive $T$ cells infiltrate the pancreas to destroy insulin producing $\beta$-cells. The persistent destruction of pancreatic $\beta$-cells is a slow process and may become undetectable until the majority (>80\%) of pancreatic islets are destroyed or rendered dysfunctional, making an individual hyperglycemic and dependent on exogenous insulin ${ }^{54,62} \cdot \mathrm{CD}^{+} \mathrm{T}$ cells are the predominant population in the insulitis region, followed by macrophages, $\mathrm{CD} 4^{+} \mathrm{T}$ cells, and B cells.

Van Belle TL et al. ${ }^{62}$ proposed that the disequilibrium between immune suppressive mechanisms (Treg cells) and autoreactive T cells occur over time and shifts the balance to islet autoimmunity, leading to significant loss of islets mass and T1D (Figure 2). $\mathrm{CD} 8^{+} \mathrm{T}$ cells mediated killing of beta cells, by release of perforin and granzyme or Fas-FasL dependent interaction, is likely the central mechanism of beta cell destruction. $\mathrm{CD}^{+} \mathrm{T}$ cells likely contributes to activation of $\mathrm{B}$ cells and $\mathrm{CD} 8^{+} \mathrm{T}$ cells by secretion of cytokines (IL-21) or by positive feedback mechanism (CD40-CD40L $)^{62,67}$. 


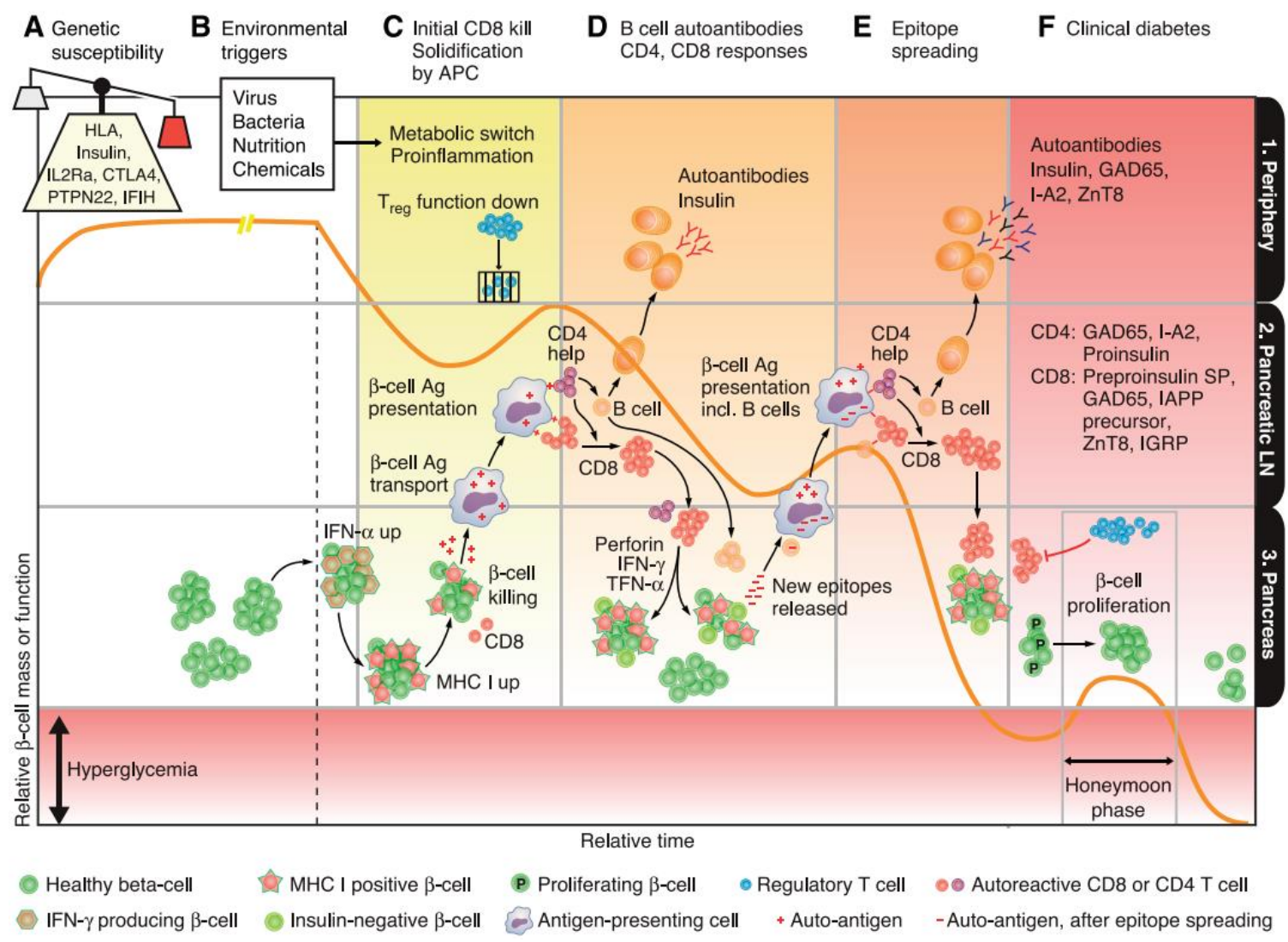

Figure 2: Progression of type 1 diabetes. (Adapted from Van Belle TL et al. 2011.)

\section{Medical management of T1D}

Once T1D is diagnosed, the main objective is to maintain the BGL near normal level. Insulin remains the mainstay of the therapy although insulin analogs are available to manage BGL. Frequent glucose monitoring and multiple dose insulin regimen is required for optimal glycemic control. Multiple insulin injections or insulin pumps have their own complications and are limited to controlling medical complications, including hyper or hypoglycemia and vascular injury ${ }^{54}$. Hypoglycemia and ketoacidosis are potentially lifethreatening complications. Genetic predisposition with serological evidence for multiple autoantibodies is enough to predict the susceptibility of T1D. Multiple preventive strategies 
that target autoimmune responses are being tested to prevent progression of T1D. Most strategies broadly implement immunomodulatory agents to maintain tolerance. Nevertheless, it is still at the primacy for prevention and reversal of T1D with no therapeutic agents being approved ${ }^{59,68,69}$. Following the breakthrough protocol for islet transplantation, the procedure has become standard of care treatment for individuals who have developed end-stage renal failure. Pancreatic islet transplantation involves infusion of donor islets into the liver via portal vein.

\section{Pancreatic islet transplantation}

Pancreatic islet transplantation (PITx) involves isolation of islets (containing $\beta$ cells along with other endocrine and non-endocrine cells) and infusion into the liver of recipient via hepatic portal vein. Since the first islet transplantation was performed two decades ago, PITx is now considered safe and real therapeutic option for patients with chronic pancreatitis (autotransplantation) or in selected patients with affected by T1D (allotransplantation) $^{70,71}$.

Chronic pancreatitis is a syndrome with progressive inflammatory condition in pancreas leading to permanent damage leading to impaired endocrine and exocrine function $^{72}$. This disease is the result of multiple environmental and genetic factors and involves replacement of pancreatic secretory parenchyma by fibrous tissue. Chronic pancreatitis is debilitating, painful, and eventually leads to diabetes. Total pancreatectomy followed by auto islet transplantation is performed to eliminate pain of pancreatitis and mitigate resultant pancreatogenic diabetes. So far more than 500 islet autotransplantation have been performed for cases of chronic pancreatitis. The procedure involves complete resection of pancreas followed by isolation of healthy islets from the diseased pancreas and 
reinfusion of isolated islets to patient via hepatic portal vein for intrahepatic engraftment without use of immunosuppressive drugs ${ }^{72,73}$.

In addition to patients with chronic pancreatitis, islet transplantation is also proposed for selected patients with other clinical indications: i) brittle T1D with unaware episodes of hyperglycemic and hypoglycemia; ii) combined organ transplantation (kidney) in T1D patients with kidney failure; iii) T1D patients under immunosuppressive therapy for other autoimmune diseases ${ }^{70}$. Pancreatic islet transplantation along with whole pancreas transplantation can restore normoglycemia as well as achieve exogenous insulin independence. However, whole pancreas transplantation is associated with significant morbidity and mortality at early transplant period. Instead pancreatic islet transplant is minimally invasive and is carried out by infusion of islets into the liver via portal vein ${ }^{74}$. With current advances, pancreatic islet transplantation is equally efficient to whole pancreas transplantation in normalizing BLG. The excellent therapeutic effect is the accurate blood glucose level responsive insulin secretion. The success of islet transplantation was realized after "Edmonton protocol" was established. The protocol uses glucocorticoid free immunosuppression and the long-term outcome results in 50-70\% of patients achieve insulin independence at 5 years. More than 1500 procedures have been performed worldwide as of date. However, limited pancreas supply and use of chronic immunosuppression are the major contributing factors that restricts the applicability of this procedure $^{75}$. 


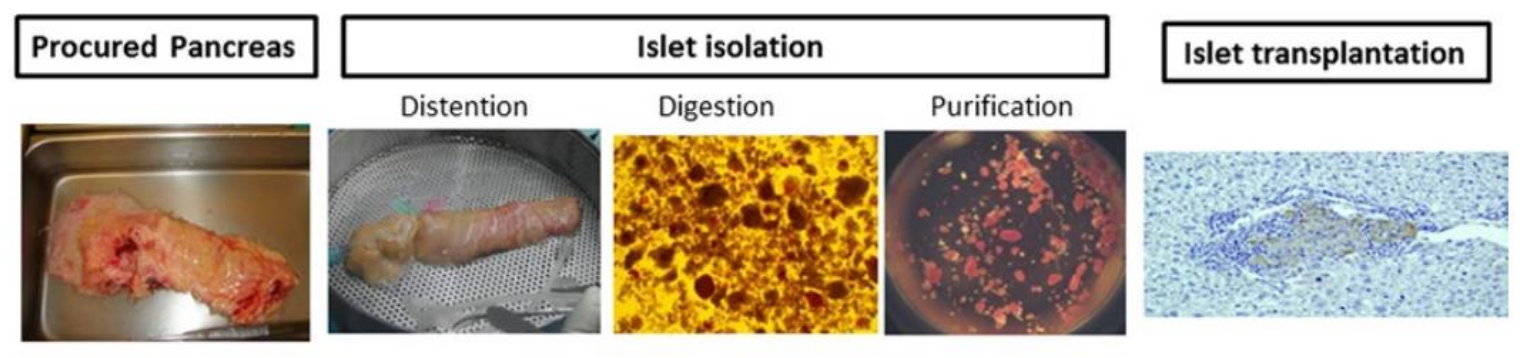

\section{Islet mass}

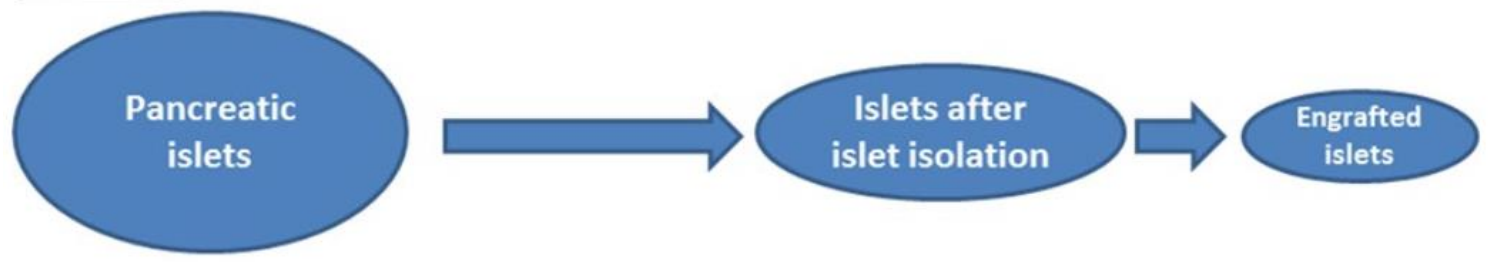

\section{$\underline{\text { Influence factors }}$}

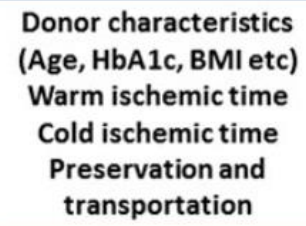

Islet isolation technique Selection of dissociation enzyme Purification method Culture conditions

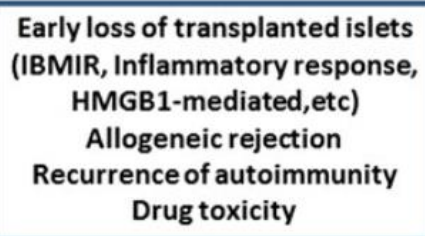
(IBMIR, Inflammatory response, HMGB1-mediated,etc) Allogeneic rejection Recurrence of autoimmunity Drug toxicity

\section{Figure 3: Pancreatic islet transplant procedure and factors contributing to loss of}

islets. (Adpated from Anazawa et al. 2019)

Transplantation into the liver via portal vein is the only clinically approved site for islets. Despite the progress in islet isolation and transplantation procedure, intraportal islet transplantation suffers from significant loss $(50-70 \%)$ of functional islet mass during the peritransplant period ${ }^{76,77}$, thus requiring islets from more than two donors to stabilize $\mathrm{BGL}^{78}$. Despite being transplanted from multiple donors, transplanted recipients have $\beta$ cell function of only $20 \%$ of that in healthy individuals ${ }^{79}$. Multiple factors contribute to this that include localized hepatic ischemia/reperfusion, low oxygenation rate, endogenous liver immune response, and instant blood mediated inflammatory reaction (IBMIR). 


\section{Instant blood mediated inflammatory reaction (IBMIR)}

IBMIR is a complex non-specific innate immune response generated by contact of islets to blood when islets are infused to liver via hepatic portal vein. IBMIR is characterized by rapid activation of coagulation and complement system, rapid activation of platelets, neutrophils and binding. This generates islet-thrombus and intra-islet infiltration of dense lymphocyte and macrophage. This cascade of inflammatory reaction ultimately leads to disrupted islet integrity, morphology and loss of islet mass ${ }^{80,81}$.

Activation of coagulation cascade and complement includes the initial steps in IBMIR. Islet isolation step involves treatment of whole pancreas with collagenase and isolation of "naked islets". This process exposes molecules (collagen, laminin) that are thrombogenic in nature. In addition, isolated islets also lack membrane regulators (heparan sulfate $)^{81,82}$. Cumulatively, the exposure of naked islets to blood facilitates activation of coagulation and complement. Further, stress during the procurement procedure, isolation and culture and brain death of donor induces pro-inflammatory signature with significant upregulation of tissue factor (TF), monocyte chemoattractant protein-1 (MCP-1), IL-1 $\beta$ and TNF- $\alpha$. Strong coagulation cascade peaks as early as 6-12 hrs post transplantation and associated with upregulated levels of different pro-coagulating factors. Sequentially, IBMIR is also associated with activation of complement. Deposition of $\operatorname{IgG}, \operatorname{IgM}$ along with C1q, C4, C3 and C9 result in formation of pro-inflammatory anaphylatoxins C3a and C5 $\mathrm{a}^{80,81,83,84}$. Activated thrombin promotes the secretion of adhesion molecule (p-selectin), thus activating platelet aggregation. This results in generation of islet-thrombus.

A panel of cytokines (IL-6, IL-8) recruit neutrophilic granulocytes and macrophages into the hepatic graft site. Under hypoxic condition, islets express pro- 
inflammatory and danger signals like high mobility group box-1 (HMGB-1), IFN- $\gamma$, IL-8, MCP-1. The intraislet macrophages, kuppfer cells, and neutrophils secret IL-1 $\beta$ that directly impairs insulin secretion and induce islet apoptosis ${ }^{85,86}$. Moberg et al. ${ }^{87}$ provides direct evidence of IBMIR mediated islet loss using in vitro loop assay. Accordingly, rapid platelets deposition occurs as early as 5 mins reaching maximum by 30 mins. Whereas $\mathrm{CD}_{11} \mathrm{~b}^{+}$neutrophilic granulocytes are predominant cell types infiltrating the islets. The cells occur as early as 15 mins and peaks by $2 \mathrm{hrs}^{87}$. Activated neutrophilic granulocytes generate reactive oxygen derivatives. ROS along with proteases liberated from the granules of activated granulocytes implement direct damage to the islets ${ }^{84,87,88}$. In addition, they secrete MCP-1 to attract macrophages. Macrophages exert killing by phagocytosis, but simultaneously also act as APCs communicating with lymphocytes in the activation phase of specific allo-immune responses ${ }^{76}$.

\section{Strategies to prevent IBMIR}

Multiple approaches have been tested to abrogate IBMIR, however all strategies focus on the coagulation cascade to prevent generation of islet-thrombus. Clinically, heparin infusion is performed to prevent coagulation. Final islet product is infused in suspension of transplantation media containing heparin (70 units per $\mathrm{Kg}$ of recipient body weight). Systemic heparin infusion is performed for $48 \mathrm{hrs}(3 \mathrm{U} / \mathrm{Kg} / \mathrm{hr})$ to prevent IBMIR ${ }^{75}$. However, Bennet et al. ${ }^{89}$ suggested, in ex vivo settings, that even using a high level of heparin $(4 \mathrm{U} / \mathrm{ml})$ was not enough to prevent extensive fibrin deposition and infiltration of $\mathrm{CD}_{11} \mathrm{~b}^{+}$cells. In addition to the risk of systemic bleeding, the relatively short half-life of systemic heparin impacts the therapeutic potential. Alternatively, Cabric et al. ${ }^{90}$ used biotin/avidin strategy to display $40 \mathrm{U}$ of heparin complexes per islet to mitigate IBMIR in 
murine and porcine models. They reported prolonged syngeneic islet graft survival when 300 islets were transplanted under the kidney capsule in a mouse model. However, under clinical settings islets are transplanted to a patient's liver via hepatic portal vein. IBMIR is only observed in intraportal settings where islets come in direct contact with recipient's blood circulation. Other anti-coagulation strategies involving low-molecular-weight dextran sulfate ${ }^{91}$ and the combination of Tirofiban and activated protein C (APC) $)^{92}$ have been reported to ameliorate IBMIR in ex vivo models. A study by Contreras et al. ${ }^{93}$ used a systemic bolus of recombinant APC to mitigate the early loss of islets. However, the use of anti-coagulating agents is always linked to high risk of bleeding and hepatic hematoma ${ }^{94,95}$. Strategies targeting anti-inflammatory ${ }^{88,96,97}$ and chemokine inhibitors ${ }^{98}$ have also been reported to mitigate the effect of IBMIR.

\section{CD47-SIRPa, an innate immune checkpoint}

CD47, also known as integrin associated protein (IAP), was identified as missing protein in Rh-hull red blood cells. The same protein was also isolated with $\alpha v \beta 3$ integrin in leukocytes and placenta ${ }^{99}$. CD47 is ubiquitously expressed by virtually all cells in the body. It interacts in cis with integrins, as well as acts as ligand for two members of signal regulatory protein (SIRP) receptor family ${ }^{100}$. CD47 is also a receptor for secreted protein thrombospondin-1 (TSP-1). CD47 is known to modulate immune responses in neutrophils, macrophages, dendritic cells and T cells ${ }^{101}$.

CD47 is a member of immunoglobulin (Ig) superfamily with heavily glycosylated IgV-like extracellular domain at its N-terminus, highly hydrophobic five putative membrane spanning transmembrane domain, and alternatively spliced C-terminal 
cytoplasmic domain. The extracellular domain is required for functional and physical interaction with integrins, TSP-1 and SIRP- $\alpha^{99,102}$. However, functional domain for enzymatic or protein interaction in the cytoplasmic tail is still at large. Signal regulatory protein (SIRP) belongs to Ig family of glycoproteins. SIRP family consists of an inhibitory receptor SIRP $\alpha$ (SHPS1), activating receptor SIRP $\beta 1$ and nonsignaling receptor SIRP $\gamma^{100,103}$. SIRP $\alpha$ is widely expressed on myeloid cells, macrophages, dendritic cells and precursor including hematopoietic stem cells. SIRP $\alpha$ is an inhibitory receptor and interact with CD47 via three Ig-like extracellular domain. It contains tyrosine based immunoreceptor tyrosine based inhibitory motif (ITIM) domain in cytoplasmic tail. Upon ligand binding, the cytoplasmic domain gets phosphorylated and mediates recruitment and activation of Src-homology domain 2 containing phosphatase SHP-1 and SHP-2 and ultimately negatively regulates intracellular signaling ${ }^{100,103}$.

CD47 modulates multiple cell activities including activation of neutrophils and platelets, transmigration of leukocytes, cell mobility and adhesion, and phagocytosis. The importance of CD47 in immunoregulation was revealed by the observation that pox virus upregulate homolog of CD47 as virulence factor to evade host immune responses ${ }^{104,105}$. 


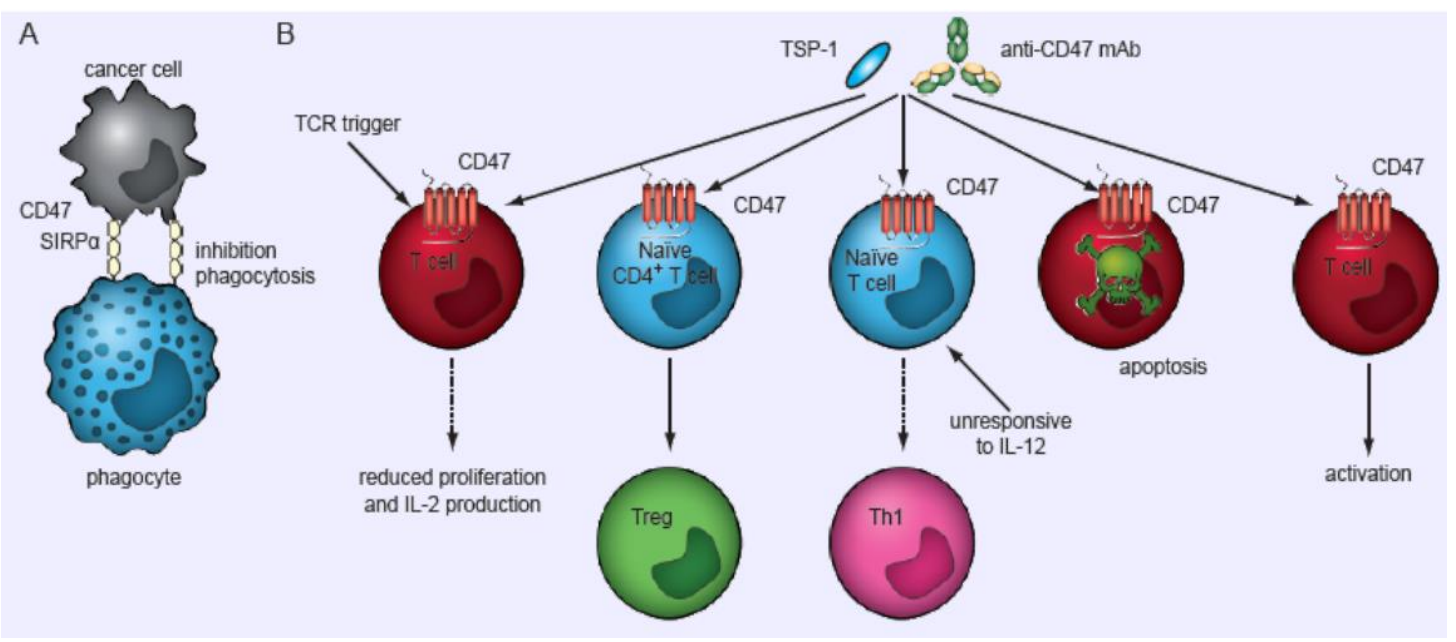

Figure 4: Multifactorial immunoregulatory role of CD47 (Wiersma, Valerie R., et al. Atlas of Genetics and Cytogenetics in Oncology and Haematology 2015.)

\section{CD47 delivers "don't eat me" signal}

One of the well-studied aspect of CD47 is "marker of self" or "don't eat me" signal delivered to macrophages and dendritic cells via $\operatorname{SIRP} \alpha^{100,106-108}$. Macrophages and dendritic cells are important regulators of innate and adaptive immune system. They constantly screen for distinction between self and non-self, such that recognition of pathogens by pathogen associated molecular patterns (PAMPs) will trigger their activation, leading to initiation of innate immune response. Interaction of CD47 as "marker of self" on healthy cells with SIRP $\alpha$ expressed on macrophages and dendritic cells results in inhibition of phagocytosis, hence coined as "don't eat me signal" ${ }^{105,109}$. Evidence of this phenomenon came from observations that CD47-deficient red blood cells (RBCs) are efficiently cleared within hours by splenic macrophages, whereas healthy RBCs have long half-lives in circulation ${ }^{110,111}$. Also, CD47 is transiently upregulated by hematopoietic stem 
cells upon mobilization or after strong inflammatory signal ${ }^{112}$. This transient upregulation is necessary for HSC to avoid being phagocytosed and cleared during their mobilization from bone marrow to blood. Studies also suggests that leukemic cells upregulate CD47 expression as an effective evasion mechanism. Increased expression of CD47 was associated with worse overall survival prognosis in patients and contributes to progression and pathogenesis of the disease due to engagement with SIRP $\alpha$ to inhibit and evade phagocytosis ${ }^{113}$.

Studies suggests expression of CD47 on DCs is important for homeostasis and migration across lymphatics and during inflammatory condition. CD47 is necessary for maturation of DCs and efficient T cells priming ${ }^{114} \cdot \mathrm{CD} 47^{-/}$murine had selective reduction of marginal zone $\mathrm{CD} 4^{+} \mathrm{DCs}$ and blunted immune responses, suggesting the important role of CD47 in DC maturation and homeostasis. Furthermore, CD47 deficient DCs had impaired migration to draining lymph nodes, despite the normal expression level of chemokine receptor ${ }^{115}$. CD47 has been reported to play a role in activation and transmigration of neutrophils and platelets. Finley et al. ${ }^{116}$ showed that blood conduits modified with recombinant CD47 inhibited activation and adhesion of platelets and neutrophils. Similar studies suggested CD47-SIRP $\alpha$ signaling can inhibit activation and mobilization of neutrophils and macrophage in CD47 adhered surfaces ${ }^{117}$. In addition, the axis is also important for antibody dependent trogocytosis, a mechanic destruction of target cell plasma membrane, of cancer cells by neutrophils ${ }^{118}$.

CD47-SIRP $\alpha$ interaction is highly species and strain specific ${ }^{100}$ and plays significant role in graft tolerance ${ }^{119,120}$. Xenografts activate recipient phagocytes due to the lack of interaction of donor CD47 with recipient SIRP $\alpha$. However, xenografts displaying 
recipient CD47 significantly diminishes phagocytic response with survival of grafts ${ }^{119,121}$. This is evidenced by significant human hematopoietic engraftment in non-obese diabetic (NOD)-severe combined immunodeficiency (SCID) mice because NOD-SIRP $\alpha$ shows enhanced binding to human $\mathrm{CD} 47^{122}$.

\section{ProtEx ${ }^{\mathrm{TM}}$ technology as a facile and effective platform for localized}

\section{immunomodulation}

Immunomodulation by definition encompasses all therapeutic or preventive interventions intended to modulate immune responses ${ }^{123}$. Multiple modalities are employed to manage immune responses based on disease condition. For instance, augmentation of immune response is desirable to combat infection, to prevent infection in states of immune deficiency, as well as to fight cancer. In contrast, in settings of transplantation, allergy, or autoimmunity strategies to dampen the immune response are

desirable ${ }^{123-125}$. Multiple drugs or therapeutic agents are often used as single agents or in combination to modulate the immune system. These agents may interfere with immune responses at different levels, antigen presentation, $\mathrm{T}$ cell activation, proliferation of regulatory cells, or induction of apoptosis of selective cell types.

In principle, the immunomodulatory biologics are used systemically, i.e., the infusion into the system by various routes of injection. This schema has significant drawback as systemically introduced biologics are rapidly cleared from the system. Alternatively, genes encoding these biologics can be introduce to target cell/tissue as an attractive platform for the treatment of various inherited and acquired immune disorders ${ }^{126}$. However, there are multiple challenges for clinical applicability of gene therapy, including 
safety issues, inefficient delivery of gene of interest, and regulation of target gene expression $^{126,127}$. As an alternative approach, our lab has pioneered the ProtEx ${ }^{\mathrm{TM}}$ technology that provides a platform to generate functional immunological ligands chimeric with core streptavidin and their transient and rapid display on biotin-modified biological surfaces for localized immunomodulation. Another major advantage of the platform is the ability to simultaneously display multiple immunomodulatory proteins for improved efficacy.

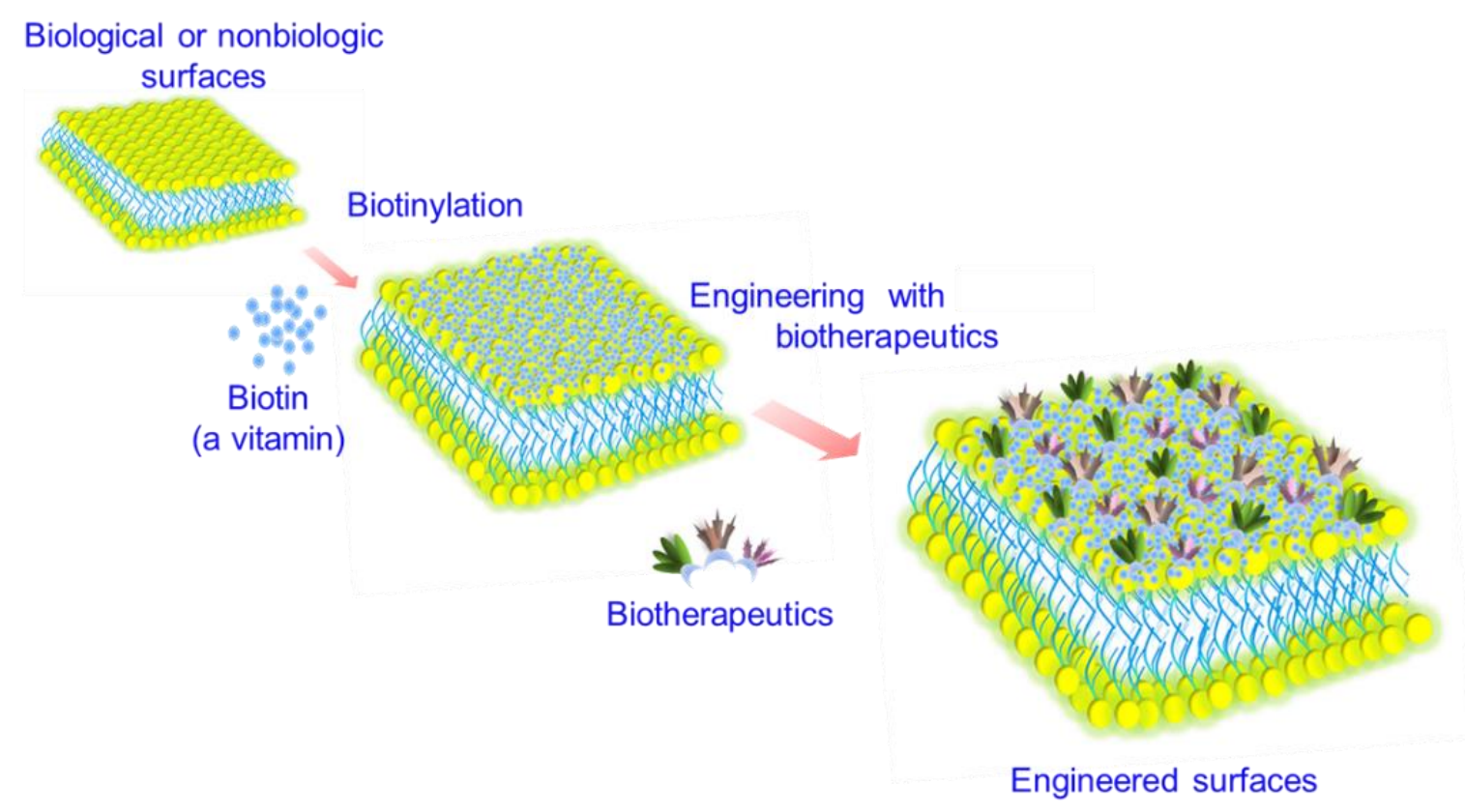

Figure 5: ProtEx ${ }^{\mathrm{TM}}$ technology to display multiple immunomodulatory proteins on the biological or nonbiological surfaces. 
Multiple functional immunomodulatory proteins, including SA-FasL, SA-PDL1, and SA-4-1BBL, have been generated using this platform and been used to induce localized tolerance to islet grafts ${ }^{128-130}$ and cardiac grafts ${ }^{131}$, efficient engraftment of stem cells ${ }^{132,133}$ and development of cancer vaccines ${ }^{134,135}$. Here, in this set of study we extend our observation as efficient immunomodulatory approach in acute GVHD and IBMIR by targeting SA-FasL and SA-CD47, respectively. 


\section{CHAPTER 2}

\section{TARGETING FAS-FASL PATHWAY FOR SELECTIVE ELIMINATION OF ALLOREACTIVE DONOR CELLS FOR PREVENTION OF ACUTE GRAFT VERSUS HOST DISEASE}

\section{Introduction}

Allogeneic hematopoietic stem cell transplantation (HSCT) is a well-established curative treatment modality for multiple high-risk hematologic malignancies as well as uncurable non-malignant hematological and genetic disorders ${ }^{2,4,51}$. However, the beneficial efficacy of this treatment is significantly limited by graft-versus-host disease (GVHD). GVHD represents a complex disease; while several factors contribute to its pathogenesis, the major mechanisms underlying the disease are well-elucidated. Interaction of $\mathrm{T}$ cell expressing suitable T cell receptor (TCR) with antigen presenting cells (APCs) that express recipient allogeneic $\mathrm{MHC}$ molecules is the driving force for the development of acute GVHD. T effector cells activated in response to recipient alloantigens expand and inflict damage to the recipient tissues. T cells are the main effectors of targeted tissue damage ${ }^{2}$. The GVHD causing alloreactive cells constitute a minor subset of total T lymphocytes ${ }^{48}$, which presents technical challenge for their complete elimination. Although, rigorous panT cell depletion from the donor graft can significantly reduce the incidence and severity of acute GVHD, the procedure is associated with multiple complications including failure in graft engraftment, leukemia relapse, and delayed immune reconstitution ${ }^{47,48,51}$. Thus, the major challenge is to control donor immune reconstitution in the recipient post- 
transplantation, conserve beneficial donor $\mathrm{T}$ cell immune function against leukemia and infections and reduce the severity of acute GVHD.

One of the distinctive features of the immune system is homeostatic control involves a phase of contraction after clonal expansion of antigen-activated lymphocytes to titrate the level back to the base line $e^{35,36,136,137}$. This is achieved by fine tuning between expansion and death triggered by apoptosis, activation induced cell death (AICD). AICD is mediated by Fas/FasL (CD95/CD95L) pathway and is an important physiologic strategy to control the expansion of antigen-activated $\mathrm{T}$ cells. AICD requires repeated antigenspecific TCR re-engagement in the presence of IL-2 and does not affect bystander T cells $^{30,52,136,138}$. Resting $\mathrm{T}$ cells do not express Fas-receptor and express multiple apoptotic inhibitors (e.g. Flice like inhibitory protein, FLICE, or surviving), whereas activated antigen-specific $\mathrm{T}$ cells upregulate Fas receptor and significantly downregulate antiapoptotic molecules ${ }^{36,52,139}$. This marked difference makes alloreactive $\mathrm{T}$ cells prone to Fas/FasL mediated apoptosis, while endowing resistant to resting or bystander T cells. In addition, multiple studies suggest that $\mathrm{HSCs}^{133,140-143}$ and immunosuppressive regulatory $\mathrm{T}$ cells ${ }^{144}$ are highly resistant to FasL-mediated AICD. The differential response of activated, naïve, regulatory $\mathrm{T}$ cells, and HSC to Fas/FasL-mediated apoptosis provides an attractive approach to use FasL to selectively eliminate alloreactive T cells without a major negative impact on HSCs as well as resting $\mathrm{T}$ cells that aid in engraftment and $\mathrm{T}$ regulatory cells that modulate alloreactive immune responses.

In our previous studies, we have reported a novel form of FasL protein chimeric with streptavidin (SA)-FasL that exist as tetramers and oligomers with potent apoptotic activity on Fas-expressing cells ${ }^{130,145}$. The protein can be efficiently and rapidly displayed 
on the surface of biotinylated cells and biologics ${ }^{128,130,133,143,145}$. Most importantly, SAFasL engineered cells or biologics could be employed to eliminate alloreactive $\mathrm{T}$ cells for systemic or localized immunomodulation ${ }^{128,130,131}$. Since upon activation alloreactive $\mathrm{T}$ cells upregulate Fas expression and become susceptible to FasL-mediated apoptosis, we hypothesized that the transient display of SA-FasL on donor cells will induce apoptosis in T cells following activation in response to the recipient alloantigens, thereby blocking acute GVHD. In line with our expectation, there was significant elimination of donor $\mathrm{T}$ cells upon allogenic stimulation in in vitro as well as in vivo settings. To test our strategy in mitigating lethal acute GVHD, we employed the haploidentical parent (C57BL/6) to F1 (C57BL/6xBALB/c) setting. We report that recipients that received SA-FasL-engineered bone marrow cells containing mature T cells were protected from lethal acute GVHD and survived long term (>100 days), whereas all controls displayed clinical signs of acute GVHD and met end point by 40 days. The long-term recipients were immunocompetent as they rejected third party, but not donor, skin grafts. Importantly, this concept also showed efficacy in a humanized mouse model for xenogeneic GVHD where human PBMC engineered with SA-FasL was used for transplantation. Thus, this approach has significant translational potential for the prevention of acute GVHD as a single modality or in combination with other clinically used approaches. 


\section{Materials and methods}

\section{Animals}

C57B1/6 $\left(\mathrm{H} 2^{\mathrm{b}}\right), \mathrm{BALB} / \mathrm{c}\left(\mathrm{H} 2^{\mathrm{d}}\right), \mathrm{C} 3 \mathrm{H}\left(\mathrm{H} 2^{\mathrm{k}}\right)$ and NOD-scid-IL2 $\gamma \mathrm{R}^{\text {null }}$ (NSG) mice were purchased from the Jackson Laboratory and breed in our specific pathogen free facility. C57BL/6.FoxP3 ${ }^{\text {hCD2}}$ (hereafter referred as B6.hCD2) ${ }^{146}$ and B6.SJL-4C.TCR-tg (hereafter referred as 4C.SJL) ${ }^{147}$ animals were generously provided by Drs. H. Waldmann of Oxford University and TV Brennan of Duke University respectively. F1 (C57BL/6 x BALB/c, $\mathrm{H} 2^{\mathrm{b} / \mathrm{d}}$ ) were breed in our facility. All animals were maintained in our specific pathogen free vivarium at the University of Louisville. All experiments were performed in accordance to approved protocols by Institutional Animal and Use Committee, University of Louisville.

\section{Modification of cell surface to engineer with SA-FasL protein}

Spleens from C57BL/6 or 4C.SJL were harvested and processed into single-cell suspension using frosted slides. Red blood cells were lysed using a home-made buffered ammonium chloride solution. SA-FasL engineering of spleen cells was done following previously reported protocols ${ }^{145}$. In brief, cells were incubated in $5 \mu \mathrm{M}$ EZ-Link ${ }^{\mathrm{TM}}$ Sulfo-NHS-LC biotin (hereafter referred to as biotin) solution (ThermoFisher Scientific, Ref\# 21335) in sterile PBS at room temperature for $30 \mathrm{~min}$. After washing with PBS, cells were incubated in PBS containing indicated amount of SA- or SA-FasL protein for $30 \mathrm{~min}$ in a cold room by constant rocking. Cells were washed before further experimental use. 


\section{MLR cultures and proliferation assay}

Standard mixed lymphocyte culture assay was performed ${ }^{128,130}$ where spleen cells from 4C.SJL was used as responders against 2000 cGy irradiated BALB/c spleen cells. Briefly, SA/SA-FasL engineered at indicated levels or unengineered 4C.SJL spleen cells were incubated at $37{ }^{\circ} \mathrm{C}$ for 45 mins to collect pan-T cells. Non-adherent pan-T cells were collected, washed and co-culture with irradiated BALB/c spleen cells $\left(10^{5}\right.$ cells each/well) in 96 well U-bottom plate in complete MLR medium ${ }^{128}$. For $\left[{ }^{3} \mathrm{H}\right]$ thymidine incorporation assay, the cultures were pulsed with $\left[{ }^{3} \mathrm{H}\right]$ thymidine $(1 \mu \mathrm{Ci} /$ well $)$ for last $16 \mathrm{hrs}$ before harvest. Cultures were then harvested with Tomtec cell harvester and analyzed in a beta plate counter to assess DNA-associated radioactivity as measure of proliferation [counts per minute (cpm)]. For flowcytometric analysis, the cells were harvested, and surface stained as described below.

For in vitro apoptosis assay, spleen cells from 4C.SJL mice were engineered with SA-FasL (100 ng). SA-FasL engineered or unmodified 4C.SJL spleen cells were fluorescently labelled with $2.5 \mu \mathrm{M}$ carboxyfluorescein succinimidyl ester (CFSE; ThermoFisher Scientific Ref\# C34554) or $2.5 \mu \mathrm{M}$ Cell trace violet (CTV; ThermoFisher Scientific Ref\# C34557), respectively, following manufacturer's instruction. Fluorescently labelled 4C.SJL pan-T cells were mixed together in equal ratio to generate 4Cmix (1:1) cell suspension. Irradiated BALB/c spleen cells were cultured with 4 Cmix cells at different indicated ratio in complete MLR medium. At indicated time points cells were harvested and analyzed for $\mathrm{H} 2 \mathrm{~K}^{\mathrm{d}-}$ cells by flow cytometry. 


\section{In vivo monitoring of adoptively transferred cells}

Spleen cells from 4C.SJL or B6.hCD2 animals were harvested and engineered with SAFasL or SA (control) proteins at indicated levels. Engineered cells were fluorescently labelled with $2.5 \mu \mathrm{M}$ cell trace violet (CTV). Each F1 recipient was intravenously injected with $5 \times 10^{6}$ CTV labelled SA- or SA-FasL-engineered 4C.SJL cells or $10 \times 10^{6} \mathrm{SA} / \mathrm{SA}$ FasL-engineered B6.hCD2 spleen cells. Animals that received 4C.SJL or B6.hCD2 spleen cells were euthanized 48 or 72 hrs post transplantation and spleen cells were analyzed by flow cytometry for donor cells.

\section{Collection of human PBMC}

Human Peripheral blood mono-nuclear cells (PBMCs) were isolated as reported before ${ }^{148}$ from healthy donors under signed informed consent approved from Institutional review board of University of Louisville. Peripheral blood was collected in heparin containing vacutainer (BD Bioscience, Ref\# 364606). PBMCs were purified and collected from buffy coats by Ficoll-paque (GE, Ref\# 17-1440-03) density centrifugation and washed by sterile PBS before further use. Engineering of human PBMCs was done as described before ${ }^{145}$.

\section{Induction of acute GVHD in the parent-to-F1 setting}

A clinically relevant haploidentical setting was used to assess lethal acute GVHD as described before with some modifications ${ }^{39}$. Female or Male F1 (H2 $\left.{ }^{\mathrm{b} / \mathrm{d}}\right)$ recipients, $10-12$ weeks old, were lethally irradiated with single dose of 1000 cGy (Gammacell 40 Exactor, 
${ }^{137} \mathrm{Cs}$ source) and 4 hrs later transplanted with $10 \times 10^{6}$ whole bone marrow cells and $20 \times 10^{6}$ whole spleen cells from female or male C57BL/6 $\left(\mathrm{H} 2^{\mathrm{b}}\right)$ mice. For engineering the graft inoculum, only whole spleen cells were engineered with SA-FasL as described above. Animals were followed twice a week for acute GVHD scoring, including five clinical parameters as explained before ${ }^{149,150}$. Each parameter received score of 0 (minimum) to 2 (maximum). A clinical GVHD score index was generated by summation of the five criteria score (maximum value $=10$ ). Animals that reached total score of $>6$ and lost body weight $>25 \%$ was considered as at the end point. All animals were supported with soaked food throughout the study.

\section{Skin transplantation}

Skin grafts were procured from tail of euthanized BALB/c $\left(\mathrm{H} 2 \mathrm{~K}^{\mathrm{d}}\right)$ donor or $\mathrm{C} 3 \mathrm{H}\left(\mathrm{H} 2 \mathrm{~K}^{\mathrm{k}}\right)$ third party mice. Each section of tail skin graft from each donor was transplanted onto prepared skin bed site of an isoflurane anesthetized long term animal. The site was covered with an adhesive bandage, which was removed after 7 days. Animals were followed post transplantation and followed until complete loss of skin grafts ${ }^{151}$.

\section{Xenogeneic GVHD protocol}

Humanized xenogeneic GVHD was induced as described before ${ }^{148}$. Briefly, 8-10 weeks old NSG females received sublethal dose (200 cGy) of total body irradiation and injected 4 hrs later with $10 \times 10^{6}$ engineered or unmodified human PBMCs via tail vein. Fresh human PBMCs were used in all experiments. Animals were followed for body weight twice a 
week and the development of xenogeneic GVHD was assessed by five parameters: loss in body weight, posture, mobility, fur texture and skin texture ${ }^{152}$. Each parameter received a score of 0 (minimum) to 2 (maximum). Animals that reached significant body weight loss (> 25\%) and total GVHD score > 6 were considered to reach end point and euthanized.

\section{Antibodies and Flow cytometry}

Fluorochrome conjugated monoclonal antibodies (Table 1 and 2) were titrated for optimal concentration before use. For cell surface staining, cells were incubated with the respective antibodies for 30 mins at $4{ }^{\circ} \mathrm{C}$. For intracellular cytokine staining, cells were stimulated with PMA (50 ng/ml) and Ionomycin $(1 \mu \mathrm{g} / \mathrm{ml})$ for $6 \mathrm{hrs}$ in presence of Golgi plug (BD Biosciences, Ref\# 51-2301KZ) for last 4 hrs. After incubation, cells were surface stained followed by fixation (eBiosciences, Ref\# 00-5123-43) and permeabilization (eBiosciences, Ref\# 00-8333-56). For intracellular staining, cells were incubated with respective cytokine antibodies for $1 \mathrm{hr}$ at room temperature. For intranuclear FoxP3 staining, Fix/Perm buffer (eBiosciences, Ref\# 00-5523-00) was used per manufacturer's instructions and staining with anti-FoxP3 antibody for 30 min at $4{ }^{\circ} \mathrm{C}$. Flow cytometric analysis was done by using LSR II with FACS Diva software. Data analysis was done by using FlowJo software (Treestar). 
Table1. Phenotypic analysis of mouse cells by flow cytometry

\begin{tabular}{|l|l|l|r|}
\hline Marker & Clone & Vendor & \multicolumn{1}{l|}{ Cat\# } \\
\hline CD3 & 500A2 & BD Biosciences & 560771 \\
\hline CD4 & RM4-5 & BD Biosciences & 557956 \\
\hline CD8 & $53-6.7$ & BD Biosciences & 557654 \\
\hline CD44 & IM7 & eBiosciences & $48-0441-82$ \\
\hline CD62L & MEL-14 & BD Biosciences & 563252 \\
\hline PD1 & RMP1-30 & eBiosciences & $48-9981-82$ \\
\hline CTLA4 & UC10-44B9 & eBiosciences & $12-1522-83$ \\
\hline CD25 & PC61.5 & eBiosciences & $25-0251-82$ \\
\hline FoxP3 & FJK-16s & eBiosciences & $53-5773-82$ \\
\hline CD45 & $30-F 11$ & eBiosciences & $45-0451-80$ \\
\hline Fc block & 93 & Biolegend & 101320 \\
\hline
\end{tabular}

Table 2. Phenotypic analysis of human cells by flow cytometry

\begin{tabular}{|c|c|c|c|}
\hline Marker & Clone & Vendor & Cat\# \\
\hline CD45 & HI30 & BD Biosciences & 564047 \\
\hline CD3 & UCHT1 & BD Biosciences & 563109 \\
\hline CD4 & L200 & BD Biosciences & 560836 \\
\hline CD8 & SK1 & BD Biosciences & 561423 \\
\hline CD25 & M-A251 & BD Biosciences & 557741 \\
\hline CD45RO & UCHL1 & BD Biosciences & 563749 \\
\hline CD197 & 150503 & BD Biosciences & 562555 \\
\hline CD95 & DX2 & BD Biosciences & 556640 \\
\hline CD127 & HIL-7R-M21 & BD Biosciences & 558598 \\
\hline CD20 & 2H7 & BD Biosciences & 560735 \\
\hline CD4 & SK3 & BD Biosciences & 566320 \\
\hline CD20 & 2H7 & BD Biosciences & 563779 \\
\hline CD16 & 3G8 & BD Biosciences & 562874 \\
\hline CD3 & UCHT1 & BD Biosciences & 560835 \\
\hline CD8 & RPA-T8 & BD Biosciences & 555369 \\
\hline CD4 & RPA-T4 & BD Biosciences & 555347 \\
\hline
\end{tabular}




\section{Cells isolation and CD25 depletion}

Single cells suspension from spleens were depleted of CD25+ cells using Miltenyi Biotec kit following manufacturer's instructions. Cells were sequentially stained with anti-CD25 PE antibody (BD Biosciences, Ref\#553866) and super-paramagnetic microbeads conjugated with monoclonal anti-PE antibodies (Miltenyi Biotec, Ref\# 130-048-801). After staining, cells were passed through LS columns (Miltenyi Biotec, Ref\# 130-042-401) and eluted cells were collected as $\mathrm{CD} 25^{-}$cell fraction. $\mathrm{CD} 25^{-}$cell fraction was then washed with PBS and followed for SA-FasL engineering as described above.

\section{RNA isolation and quantitative RT-PCR}

Total RNA was extracted from liver, colon and small intestine tissues using Trizol reagent (Life Technologies, Ref\# 15596018) according to manufacturer's instruction. Total RNA was quantified using NanoDrop ND-2000c spectrophotometer (ThermoFisher Scientific) and cDNA was reverse transcribed from $4 \mu \mathrm{g}$ of the total RNA using SuperScript ${ }^{\mathrm{TM}}$ IV VILO $^{\mathrm{TM}}$ Master Mix (ThermoFisher Scientific, Ref\# 11756050) following manufacturer's instruction. Quantitative RT-PCR was performed for different genes (Table 3) using TaqMan probe assay on Quant Studio 3 RT-PCR system (Applied Biosystems). Each PCR reaction consisted of $5 \mu \mathrm{l}$ of TaqMan Fast Advanced master mix, $1 \mu$ l of TaqMan primer and FAM/VIC labelled MGB probes, $1 \mu \mathrm{l}$ of cDNA sample (from 1:10 dilution), and $3 \mu 1$ of nuclease-free water in a final volume of $10 \mu \mathrm{l}$. The thermal cycling conditions were as follows: 1 cycle of $95^{\circ} \mathrm{C}$ for 20 s, followed by 40 cycles of $95^{\circ} \mathrm{C}$ for $1 \mathrm{~s}$ and $60{ }^{\circ} \mathrm{C}$ for $20 \mathrm{~s}$. Transcript expression was normalized to GAPDH housekeeping gene and represented as fold change $\left(2^{-\Delta \Delta \mathrm{Ct}}, \Delta \Delta \mathrm{Ct}=\Delta \mathrm{Ct}_{\text {experimental }}-\Delta \mathrm{Ct}_{\mathrm{control}}\right)$. 


\section{Table 3. TaqMan assay for indicated gene expression in tissues}

\begin{tabular}{|l|l|}
\hline Taqman Primer & $\begin{array}{l}\text { Assay ID } \\
\text { (Thermofisher) }\end{array}$ \\
\hline CCl2/ MCP1 & Mm00441242_m1 \\
\hline IL-1 $\beta$ & Mm00434228_m1 \\
\hline FoxP3 & Mm00475162_m1 \\
\hline GATA3 & Mm00484683_m1 \\
\hline TNF- $\alpha$ & Mm99999068_m1 \\
\hline IFN- $\gamma$ & Mm01168134_m1 \\
\hline IL-4 & Mm00445259_m1 \\
\hline IL-6 & Mm00446190_m1 \\
\hline IL-10 & Mm01288386_m1 \\
\hline Rorc/ ROR- $\gamma$ T & Mm01261022_m1 \\
\hline TGF- $\beta 1$ & Mm01178820_m1 \\
\hline Tbx21/ T-Bet & Mm00450960_m1 \\
\hline GAPDH & Mm99999915_g1 \\
\hline IL-12b/ IL-12 p40 & Mm01288989_m1 \\
\hline IL-23 $\alpha$ & Mm00518984_m1 \\
\hline
\end{tabular}

\section{Histopathology}

Tissues from animals on day 21 after HSCT were harvested, fixed in $10 \%$ neutral buffered formalin (Leica, Ref\# 3800602) and embedded in paraffin. Sections (5 $\mu \mathrm{m})$ were cut with microtome and subsequently stained with hematoxylin and eosin.

\section{Statistics}

Statistical analysis was performed using GraphPad prism software v.8 (Graphpad prism, CA). Comparison of the survival curves was done using log-rank (Mantel-Cox) test. Data are shown as individual data points or as mean \pm SEM as depicted in the figure legends. Comparison of the means was performed using unpaired, two tailed test or Man-Whitney t-test as indicated. For multiple comparison, one-way ANOVA with tukey posttest was done. Statistical significance was defined as $\mathrm{p}<0.05$. 


\section{Results}

\section{T effector cells engineered with SA-FasL are efficiently eliminated in response to alloantigens}

Alloreactive T cells upregulate Fas receptor as early as 1-2 days post allo-mixed lymphocyte reaction (MLR) assay rendering them sensitive to FasL by day $2^{51}$. To test if SA-FasL engineered alloreactive cells will have reduced proliferation upon allogeneic stimulation, we performed $\left[{ }^{3} \mathrm{H}\right]$ thymidine incorporation assay. Spleen cells from $4 \mathrm{C}$ mice, TCR-transgenic on C56BL/6.SJL (CD45.1) background, were used as responders to irradiated BALB/c spleen cells. The $\mathrm{T}$ cells in $4 \mathrm{C}$ has selective reactivity against BALB/c H2-I-A ${ }^{d}$ alloantigen, which is widely expressed in mouse tissues ${ }^{147}$. Spleen cells from $4 \mathrm{C}$ were engineered with SA-FasL (SA-FasL-4C) at different levels or with control protein SA (SA-4C). SA-FasL-4C cells or SA-4C cells or unmodified (4C) cells were co-cultured with irradiated BALB/c cells. There was robust proliferation of $4 \mathrm{C}$ or SA-4C cells by 48 and 72 hours post culture observed by significant incorporation of $\left[{ }^{3} \mathrm{H}\right]$ thymidine. In contrast, we observed significant inhibition of proliferation of SA-FasL-4C cells at all protein concentrations tested (Fig. 6A). This demonstrate that SA-FasL on the surface of alloreactive T cells is extremely effective, even at the lowest protein concentration tested, in blocking their proliferation in an in vitro allo-MLR setting.

To provide evidence that SA-FasL targets both $\mathrm{CD}^{+}$and $\mathrm{CD}^{+} \mathrm{T}$ cells for inhibition, we assessed the frequency of these cells using Abs to CD4, CD8, and V $\beta 13$ TCR specific for the target alloantigen in flow cytometry. There was significant reduction in frequency of alloreactive $\mathrm{CD}^{+}$and $\mathrm{CD}^{+} \mathrm{T}$ cells expressing TCR V $\beta 13$ in the SA-FasL engineered group as compared with controls (Fig. 6B). 
SA-FasL on the surface can induce apoptosis in activated alloreactive $\mathrm{T}$ effector cells in three different ways; i) autocrine where SA-FasL engages Fas on the same T cell, ii) paracrine where SA-FasL on a $\mathrm{T}$ cell engages Fas on another $\mathrm{T}$ cell, or iii) combination of autocrine and paracrine. To investigate the relative contribution of these death pathways we performed co-culture MLR studies. SA-FasL engineered 4C and unmanipulated 4C cells were labelled with CFSE and CTV, respectively. Fluorescence labelled 4C cells were then mixed at 1:1 ratio and used as responders at indicated ratios with a fixed number of irradiated $\mathrm{BALB} / \mathrm{c}$ cells as stimulators. Unmanipulated $4 \mathrm{C}$ cells underwent robust proliferation by $72 \mathrm{hrs}$ with distinct daughter cell generations. In marked contrast, there was minimal daughter cell generation in SA-FasL-4C cells at all cell ratios used (Fig. 6C), suggesting that SA-FasL-4C cells were primarily being eliminated by autocrine apoptosis.

A

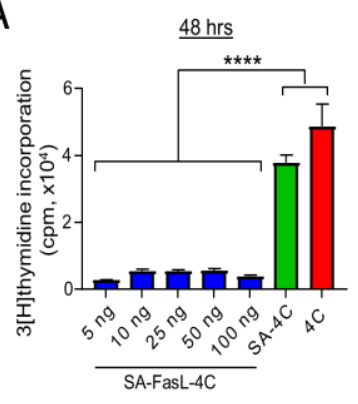

C

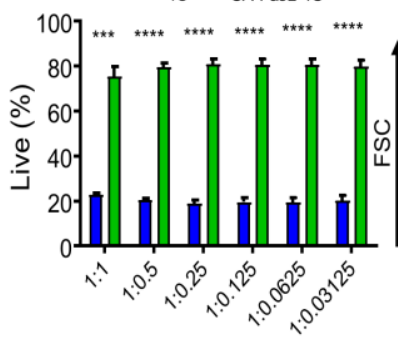

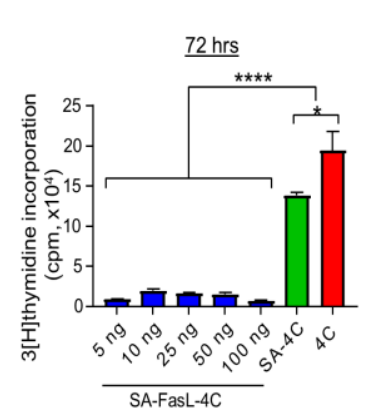
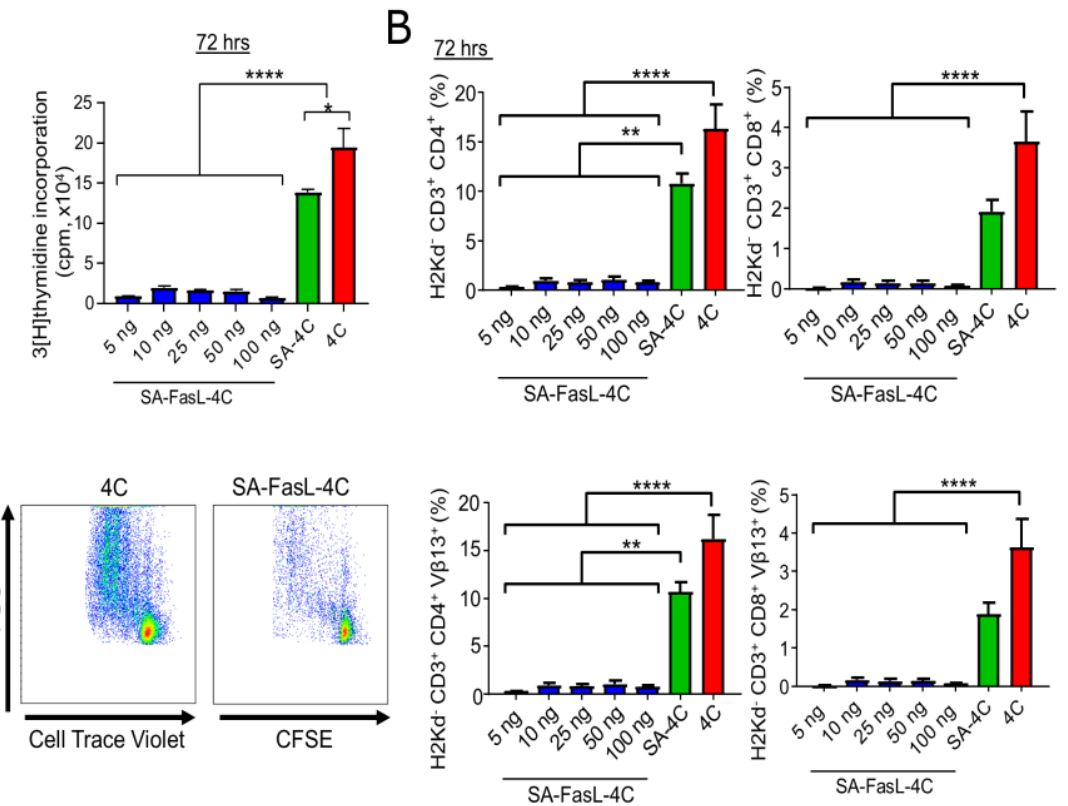

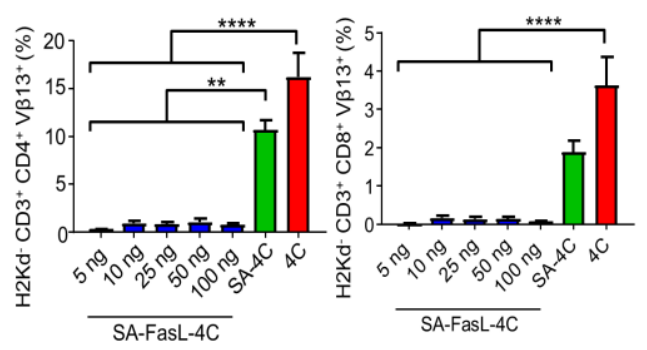


Figure 6. SA-FasL transiently displayed on the surface of $T$ cells is effective in blocking their proliferation in response to alloantigens in vitro. (A) In vitro proliferation assay. SA-FasL-engineered or unmodified 4C T cells were stimulated with irradiated BALB/c splenocytes for 48 or $72 \mathrm{hrs}$. Cultures were pulsed with $\left[{ }^{3} \mathrm{H}\right]$ thymidine for the last 16 hrs of incubation and harvested using a beta plate counter. Cell associated radioactivity was measured using a scintillation counter. Data were pooled from two independent experiments. (B) Frequencies of live CD4, CD8, and V $\beta 13 \mathrm{~T}$ cell subpopulations in mixed lymphocyte cultures. Experimental conditions are the same as in (A), except instead of pulsing with $\left[{ }^{3} \mathrm{H}\right]$ thymidine, cultures were harvested at $72 \mathrm{hrs}$, stained with the Abs to indicated markers and analyzed using flow cytometry. Data were pooled from two independent experiments. (C) SA-FasL induces autocrine death in alloreactive T cells. CTV labelled unmodified 4C cells were mixed one-to-one ratio with CFSE labelled SA-FasL-4C cells and used as responders at the indicated ratios against a fixed number of irradiated BALB/c cells as stimulators. Cells were harvested after $72 \mathrm{hrs}$ of incubation and analyzed for live cells using flow cytometry. Representative flow dot plots of proliferating 4C cells. Date sets pooled from two independent experiments. Oneway ANOVA with Tukey multiple comparison was used in (A) and (B). Unpaired two tailed t-test was used in (C). Data are shown as mean \pm SEM. cpm counts per minute. $* * p$ $<0.01, * * * p<0.001, * * * * \mathrm{p}<0.0001$ 
To recapitulate in vitro findings in an in vivo model, we used 4C.SJL (CD45.1) transfer to F1 transgenic recipients where $4 \mathrm{C}$ cells are expected to respond to the recipient H2-I-A ${ }^{\mathrm{d}}$ antigen. 4C.SJL spleen cells were modified with biotin followed by engineering with SA-FasL (100 ng/10 cells) or SA (50 ng/10 cells) as the control protein. Each F1 animal was adoptively transferred with $5 \times 10^{6}$ CTV labelled SA-FasL-4C or SA-4C cells. After 48 hrs of transfer, spleen from F1 recipients were harvested and analyzed for CD45.1 ${ }^{+}$ donor cells by flow cytometry. F1 recipients that were transferred with SA-FasL-4C cells had significantly less frequency and absolute numbers of live total donor cells (7AAD CD45. $1^{+}$) and CD45. $1^{+} \mathrm{CD} 4^{+} \mathrm{T}$ cells (Fig. 7A and 8A). SA-FasL-4C recipients had 3.8fold reduction in live donor cells and 5.7-fold reduction in live $\mathrm{CD}^{+}$donor cells as compared with SA-4C recipients. In addition, substantially less proliferated donor cells $\left(\mathrm{CTV}^{\text {low }} \mathrm{CD} 45.1^{+}\right)$were observed in SA-FasL-4C as compared with SA-4C recipients (Fig. 7A and 8A). This was evident by distinct daughter cell generations in SA-4C, but not in SA-FasL-4C, group (Fig. 8B). Annexin V staining revealed more apoptotic cells in SAFasL-4C as compared with SA-4C recipients (Fig. 7A), providing in vivo evidence for alloreactive T cells undergoing apoptosis.

To further corroborate our in vivo observation, we performed this in vivo tracking study using a non-TCR transgenic model using spleen cells from C57B/6.hCD2 (H-2K $\left.{ }^{\mathrm{b}}\right)$ donors. These mice are transgenic for human CD2 expressed under the FoxP3 promotor of mice, providing a convenient means of tracking Treg cells using an Ab to human CD2 ${ }^{146}$. Cells were engineered at indicated doses of SA-FasL or SA proteins, labelled with CTV, and $10 \times 10^{6}$ cells were injected intravenously into $\mathrm{F} 1\left(\mathrm{H} 2 \mathrm{~K}^{\mathrm{b} / \mathrm{d}}\right)$ recipients. Animals were euthanized at $72 \mathrm{hrs}$ and splenocytes were subjected to flow analysis. There was 
significantly reduced frequency (Fig. 7B) as well as absolute number (Fig. 8C) of live donor total $\left(\mathrm{H}_{2} \mathrm{~K}^{\mathrm{d}-} \mathrm{CTV}^{+}\right)$cells, $\mathrm{CD} 4^{+}$and $\mathrm{CD}^{+} \mathrm{T}$ cells as well as proliferating $\left(\mathrm{H} 2 \mathrm{~K}^{\mathrm{d}-}\right.$ $\mathrm{CTV}^{\text {low }}$ ) donor T cells (Fig. 7B and 8C) at $72 \mathrm{hrs}$ post transfer in F1 recipients of SA-FasL engineered cells as compared with those that received SA engineered cells. Interestingly, there was also a significant drop in the frequency and absolute numbers of $\mathrm{T}$ regulatory cells $\left(\mathrm{hCD} 2^{+}\right.$) in SA-FasL-spleen recipients (Fig. 7B and 8C). Importantly, these effects were observed for almost all SA-FasL protein doses tested, demonstrating the apoptotic efficacy of SA-FasL plausibly due to its autocrine mode of action.

Taken together, these findings demonstrate that the transient display of SA-FasL protein on T cells is an efficient strategy of purging out pathogenic alloreactive cells with great potential to mitigate acute GVHD. 

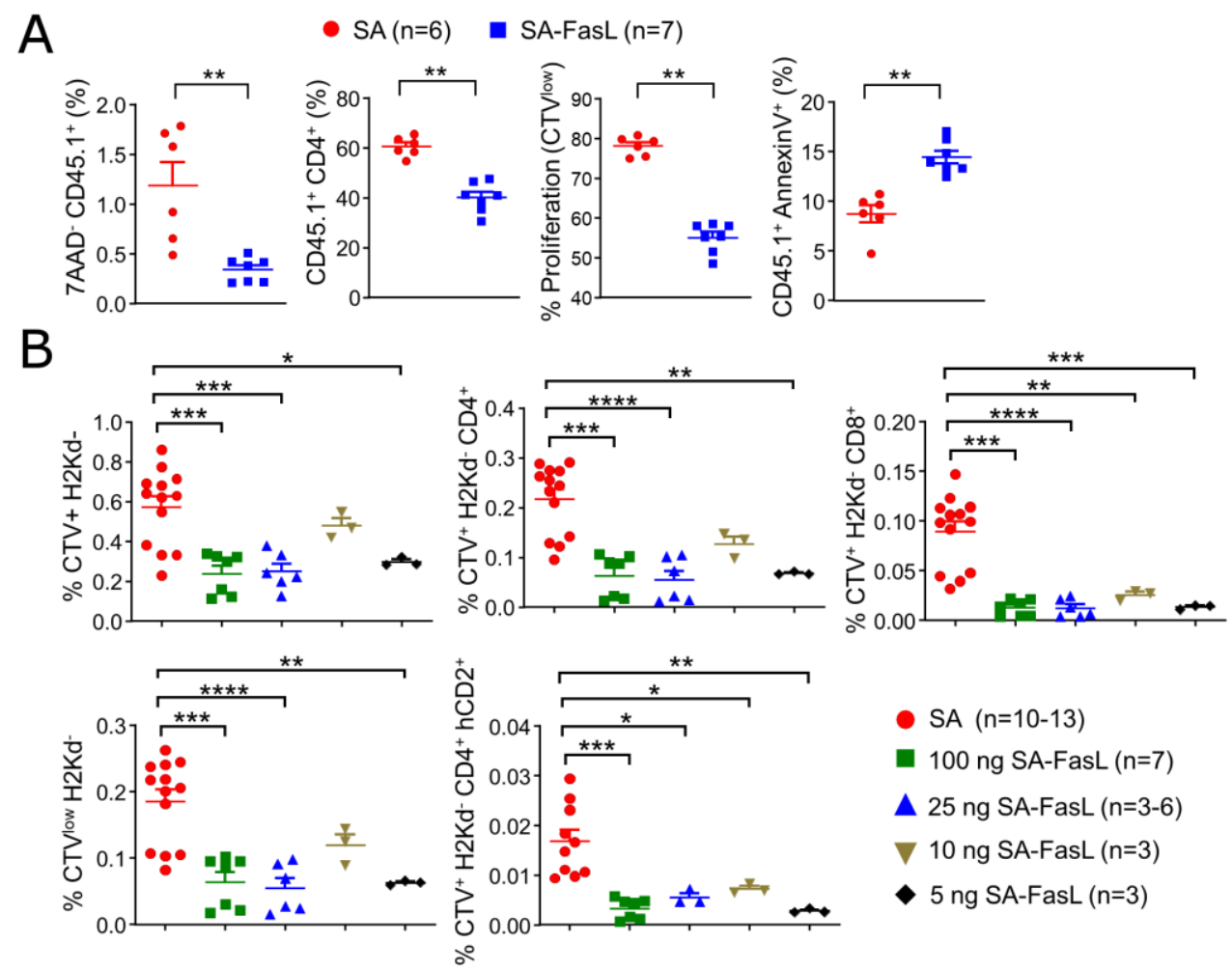

- $S A(n=10-13)$

100 ng SA-FasL $(n=7)$

$\Delta 25$ ng SA-FasL (n=3-6)

$\nabla 10 \mathrm{ng}$ SA-FasL $(\mathrm{n}=3)$

- 5 ng SA-FasL $(\mathrm{n}=3)$

Figure 7. Elimination of alloreactive cells by SA-FasL engineering in an allogeneic adoptive transfer model. (A) Tracking frequency, proliferation, and apoptosis of 4C cells in F1 recipients. 4C.SJL cells were labelled with CTV and engineered with SA or SAFasL proteins and adoptively transferred into F1 recipients $\left(5 \times 10^{6}\right.$ cells/mouse). After 48 hrs post-transplant, spleen cells of recipients were analyzed for frequency of donor total live $\left(7 \mathrm{AAD}^{-} \mathrm{CD} 45.1^{+}\right)$cells, $\mathrm{CD} 4^{+} \mathrm{T}\left(\mathrm{CD} 45.1^{+} \mathrm{CD} 4^{+}\right)$cells, proliferating $\left(\mathrm{CTV}^{\text {low }}\right)$ cells, and apoptotic $\left(\mathrm{CD} 45.1^{+}\right.$AnnexinV $\left.\mathrm{V}^{+}\right)$cells. Data pooled from two independent experiment, with $n=3-4 /$ group. (B) Tracking B6.hCD2 donor cells in F1 recipients. B6.hCD2 splenocytes were labelled with CTV and engineered with SA (50 ng/10 cells) or the indicated amounts of SA-FasL protein. Cells were adoptively transferred into F1 recipients $\left(10 \times 10^{6}\right.$ cells/mouse) that were euthanized $72 \mathrm{hrs}$ later to harvest the spleen. Splenocytes were analyzed in flow cytometry by gating on donor cells $\left(\mathrm{H}-2 \mathrm{~K}^{\mathrm{d}-}\right)$ for the frequency of 
total cells $\left(\mathrm{CTV}^{+} \mathrm{H}-2 \mathrm{~K}^{\mathrm{d}-}\right), \mathrm{CD}^{+}\left(\mathrm{CTV}^{+} \mathrm{H}-2 \mathrm{~K}^{\mathrm{d}-} \mathrm{CD}^{+}\right), \mathrm{CD}^{+}\left(\mathrm{CTV}^{+} \mathrm{H}-2 \mathrm{~K}^{\mathrm{d}-} \mathrm{CD}^{+}\right)$, and Treg $\left(\mathrm{CTV}^{+} \mathrm{H}-2 \mathrm{~K}^{\mathrm{d}-} \mathrm{CD} 4^{+} \mathrm{hCD} 2^{+}\right)$cells as well as proliferating donor cells $\left(\mathrm{CTV}^{\text {low }} \mathrm{H}_{-}-\mathrm{K}^{\mathrm{d}-}\right)$. Data pooled from three independent experiments with $n=3-4$ /group. For comparison of mean, Mann Whitney test was used in (A), One-way ANOVA with Tukey post test was used in (B). Data represented as mean \pm SEM. $* \mathrm{p}<0.05 * * p<0.01$, *** $p<0.001$, **** $\mathrm{p}<0.0001$ 

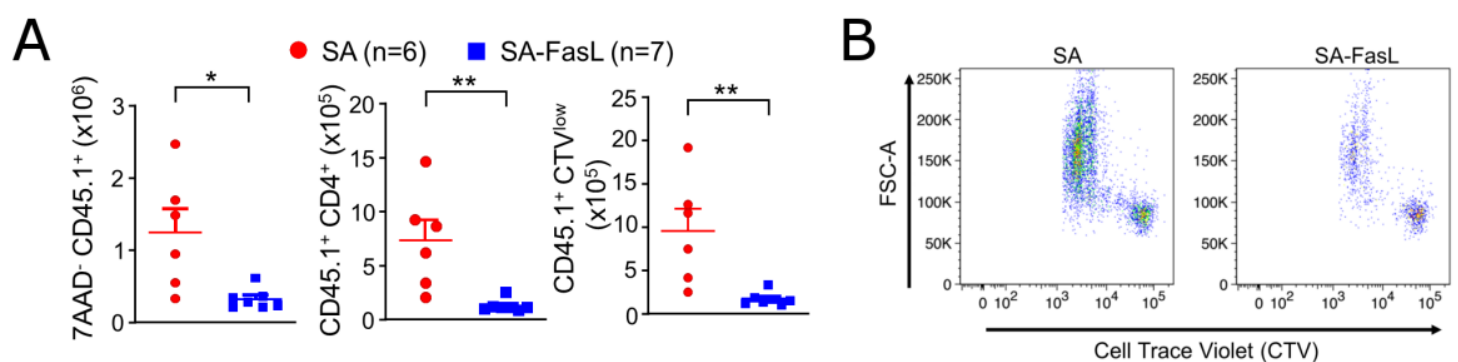

C
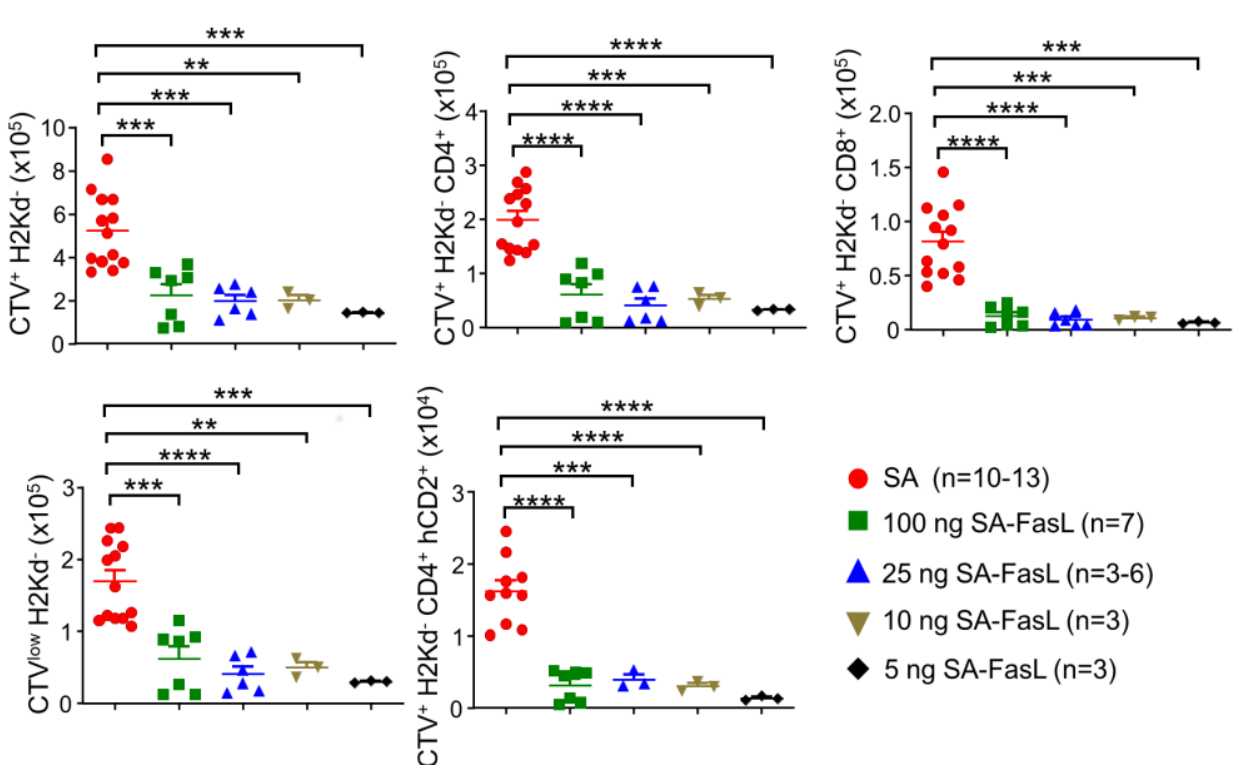

- SA $(n=10-13)$

100 ng SA-FasL $(\mathrm{n}=7)$

\ 25 ng SA-FasL $(\mathrm{n}=3-6)$

$\nabla 10$ ng SA-FasL $(\mathrm{n}=3)$

- 5 ng SA-FasL $(n=3)$

Figure 8. SA-FasL engineered alloreactive cells are eliminated in in vivo model (A) Absolute number of $4 \mathrm{C}$ cells shown in Fig. 8A. Data pooled from two independent experiments. (B) Representative flow plot showing 4C cell proliferation, equal number of live events were acquired. (C) Absolute number of B6.hCD2 donor cells subpopulations shown in Fig. 8B. Data pooled from three independent experiments with $n=3-4 /$ group. For comparison of mean, Mann Whitney test was used in (A), One way ANOVA with Tukey post test was used in (C). Data represented as mean \pm SEM. *p $<0.05$, **p $p<0.01$, ***p $<$ $0.001, * * * * \mathrm{p}<0.0001$ 


\section{Transient display of SA-FasL on donor T cells Engineering of donor graft to display}

\section{SA-FasL efficiently prevents lethal acute GVHD}

Selective depletion of alloreactive donor cells from graft is an efficient strategy for prevention of lethal acute $\mathrm{GVHD}^{2,153}$. Given the significant depletion of alloreactive T cells by engineering of cells to display SA-FasL on their surface, we assessed the efficacy of this strategy to prevent lethal acute GVHD. We used haploidentical parent (C57BL/6, $\left.\mathrm{H} 2 \mathrm{~K}^{\mathrm{b}}\right)$ to $\mathrm{F} 1\left(\mathrm{C} 57 \mathrm{BL} / 6 \mathrm{xBALB} / \mathrm{c}, \mathrm{H} 2 \mathrm{~K}^{\mathrm{b} / \mathrm{d}}\right)$ model, simulating the broad use of haploidentical bone marrow cells in the clinic. Recipient F1 animals were lethally irradiated at $1000 \mathrm{cGy}$ and $4 \mathrm{hrs}$ later were infused with $20 \times 10^{6}$ SA-FasL engineered or unmodified spleen cells mixed with $10 \times 10^{6}$ unmodified whole bone marrow cells. Controls that received only bone marrow cells survived long-term without any signs of GVHD as expected. However, recipients of a mixture of bone marrow cells and unmodified $\mathrm{T}$ cells developed acute GVHD symptoms, including bodyweight loss, hunching, and diarrhea, and expired with a median survival time of 26 days (Fig. 9A). The survival of recipients transplanted with SA-engineered donor spleen cells (SA group) and the severity of GVHD were not significantly different from the control group (MST = 25 days; Fig. 9A). The efficacy of SA-FasL was dose dependent as animals receiving T cells engineered with 25 ng SA-FasL per $10^{6}$ cells showed better survival at $>100$ days than recipients transplanted with cells engineered with 10 or 5 ng protein $(\sim 73 \%, \sim 63 \%$, and $20 \%$, respectively, Fig. 9A). Clinical GVHD scores for these groups correlated with the survival rates. Taken together, these results demonstrate the efficacy of this engineering platform with SA-FasL in preventing acute GVHD in a clinically relevant model. 
We next analyzed long-term animals for immune composition. Long term animals (>100 days) that received SA-FasL engineered grafts had full donor chimerism (Fig. 10). Immune cells in spleen, peripheral blood and bone marrow were donor derived $\left(\mathrm{H}-2 \mathrm{~K}^{\mathrm{b}+}\right.$, $\mathrm{K}^{\mathrm{d}}$ ), suggesting efficient engraftment of donor stem cells. Importantly, the frequency and absolute number of immune cells, including Treg cells $\left(\mathrm{CD} 4^{+} \mathrm{CD} 25^{+} \mathrm{FoxP} 3^{+}\right)$, $\mathrm{T}$ effector cells $\left(\mathrm{CD} 4{ }^{+} \mathrm{CD} 44^{\mathrm{hi}} \mathrm{CD} 62 \mathrm{~L}^{-}\right)$, $\mathrm{NK}$ cells $\left(\mathrm{NK} 1.1^{+} \mathrm{CD} 3^{-}\right)$as well as ratios between Treg and Teff cells in the spleen of long term animals were comparable to the BM control group that did not receive splenocytes (Fig. 9B and 11A). Similar observations were also made with peripheral blood lymphocytes (Fig. 11B). These results demonstrate that long term animals have efficient overall lymphoid reconstitution. 

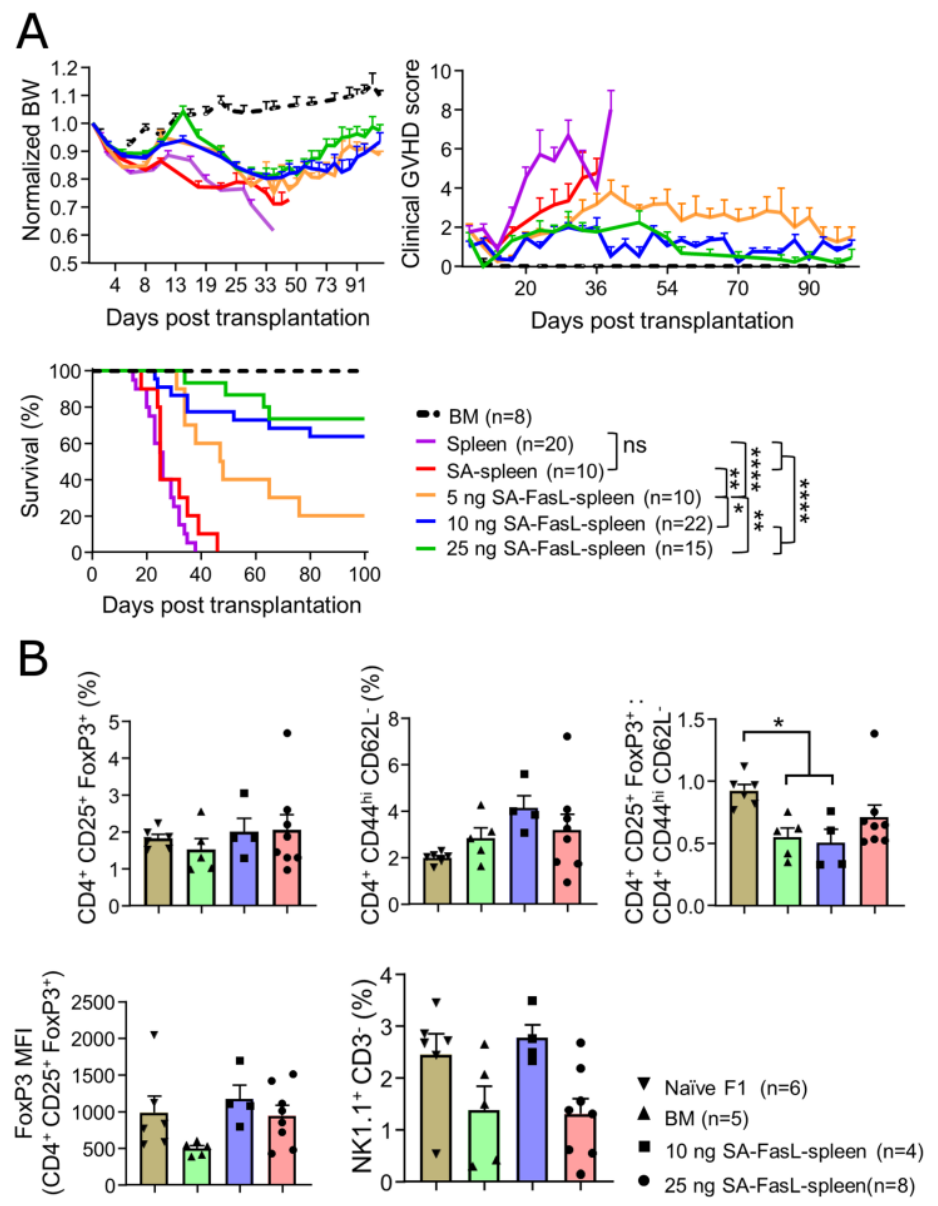

Figure 9. Engineering donor graft with SA-FasL abrogates lethal acute GVHD and show efficient lymphoid reconstitution. (A) Survival of F1 recipients transplanted with a mixture of allogeneic bone marrow and splenocytes. Lethally irradiated F1 animals were transplanted with C57BL/6 bone marrow cells $\left(20 \times 10^{6}\right)$ co-mixed with syngeneic spleen cells $\left(10 \times 10^{6}\right)$ engineered with SA $\left(12.5 \mathrm{ng} / 10^{6}\right.$ cells $)$ or the indicated amount of SA-FasL. Animals transplanted with bone marrow only (BM) or a mixture of unmodified splenocytes and bone marrow cells (Spleen) served as controls. Animals were followed for body weight, clinical GVHD scores and survival. (B) Frequency of CD4 ${ }^{+}$Treg cells, Tem and NK cells in long term (>100 days) animals compared with bone marrow only recipients and unmanipulated naïve F1 animals. For comparison of survival curves log-rank (Mantel- 
cox) test was used in (A). For comparison of means in (B), one-way ANOVA with Tukey posttest was used. Data represented as mean \pm SEM. *p $<0.05 * * p<0.01$, ***p $<0.001$, $* * * * \mathrm{p}<0.0001$
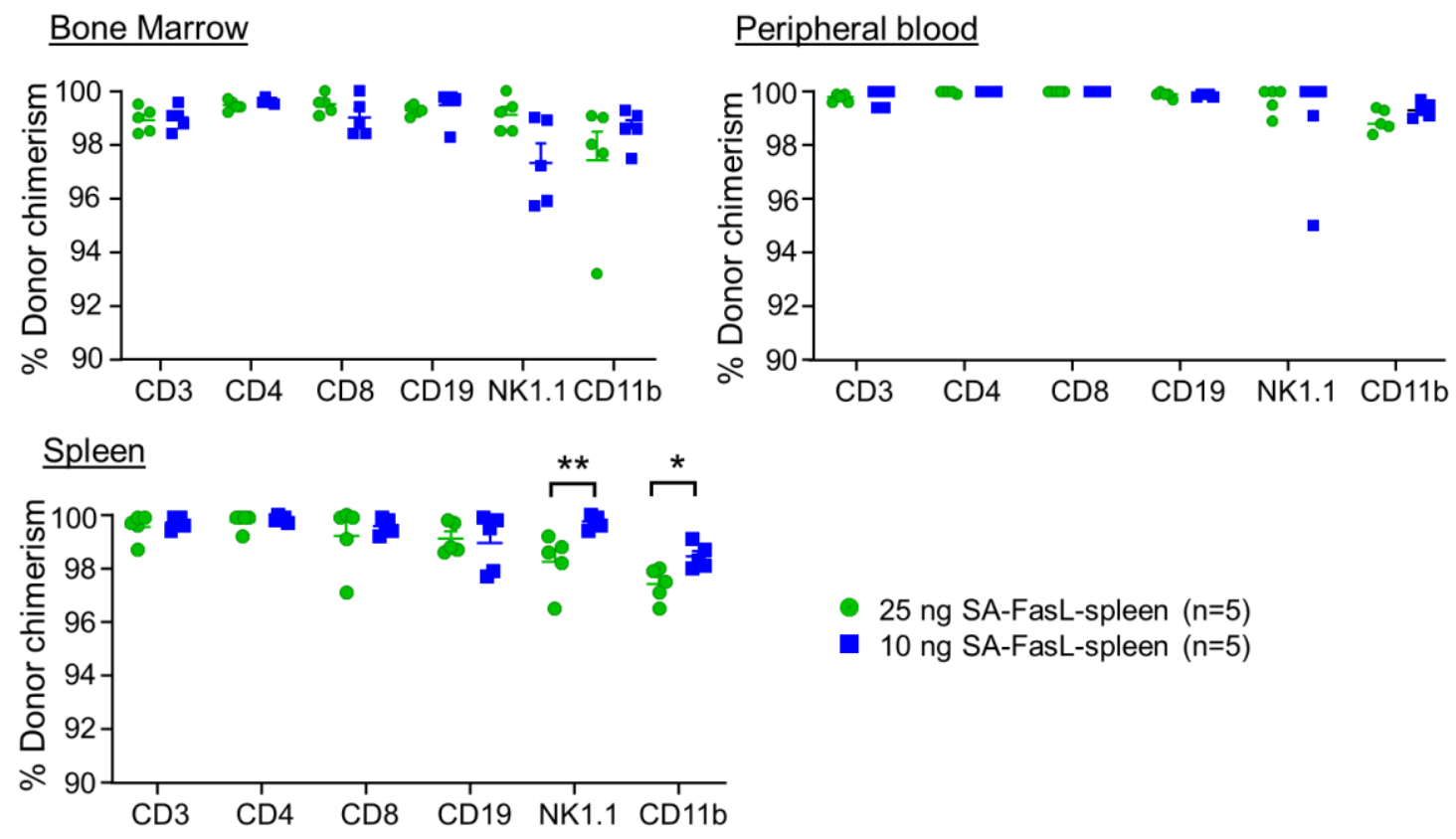

Figure 10. Long term SA-FasL-spleen recipients show full donor chimerism. Long term animals (>100 days) were analyzed for donor chimerism $\left(\mathrm{H} 2 \mathrm{~K}^{\mathrm{b}}\right.$ vs $\left.\mathrm{H} 2 \mathrm{~K}^{\mathrm{d}}\right)$ in bone marrow, peripheral blood and spleen. Frequency of $\mathrm{H}_{2} \mathrm{~K}^{\mathrm{d}-}$ (donor cells) in each cell compartment was analyzed by flow cytometry. Data shown as mean \pm SEM. Mann Whitney test was used for mean comparison. ${ }^{*} \mathrm{p}<0.05, * * \mathrm{p}<0.01$ 
A Spleen (Absolute number)

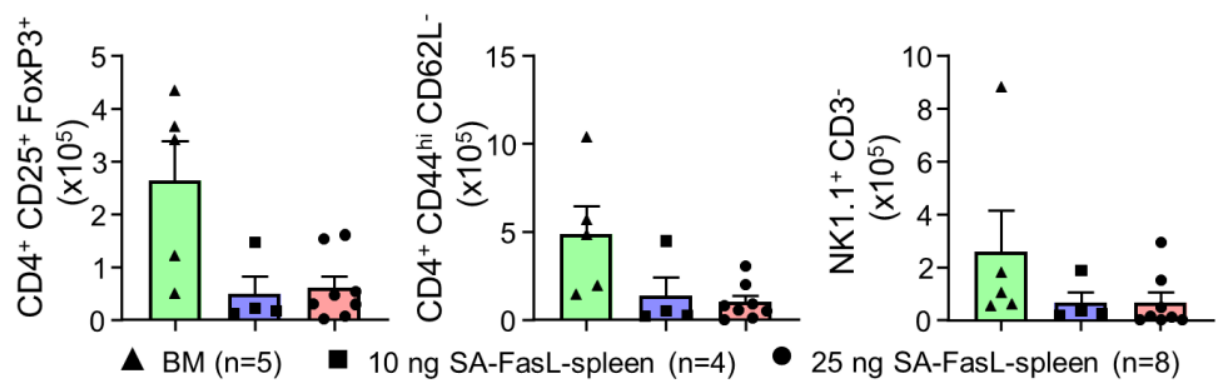

B Peripheral blood
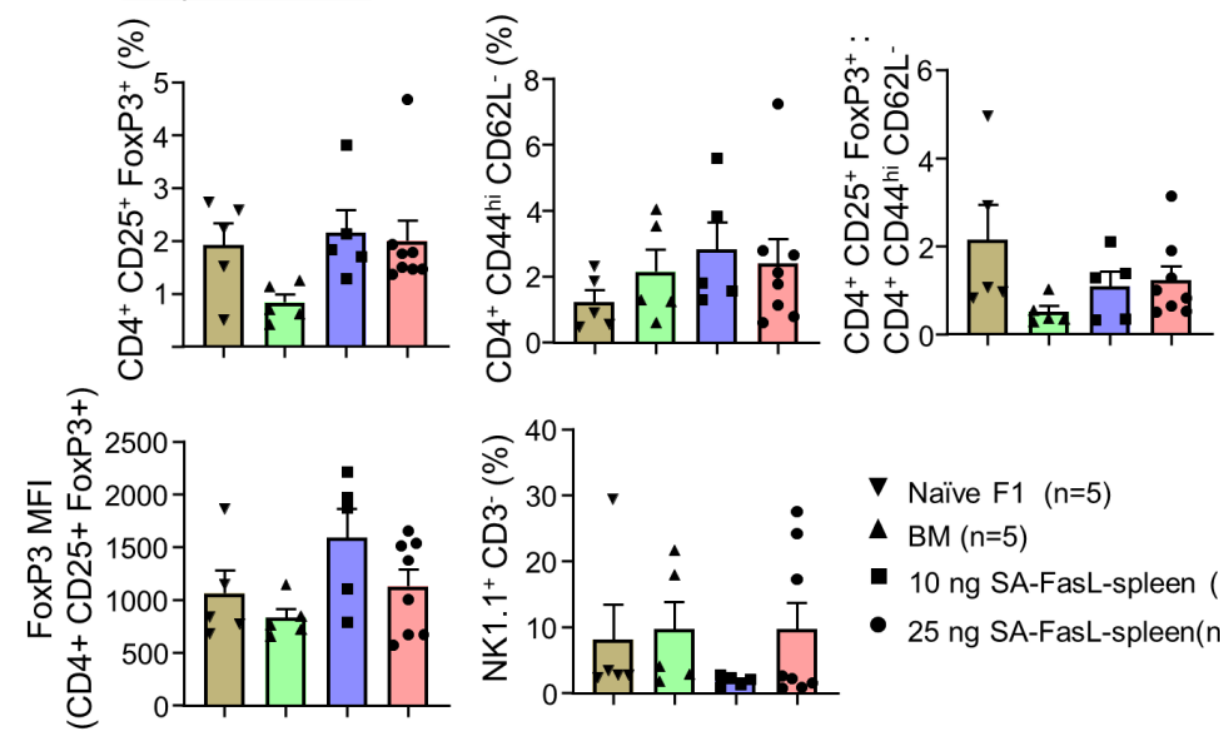

V Naïve F1 (n=5)

$\Delta B M(n=5)$

- 10 ng SA-FasL-spleen $(\mathrm{n}=4)$

- 25 ng SA-FasL-spleen $(n=8)$

Figure 11. Immunophenotyping analysis of long-term animals. Absolute number in spleen (A) and frequency in peripheral blood (B) of long-term recipients (>100 days) of SA-FasL-spleen cells compared with long-term recipients of bone marrow cells without GVHD causing spleen cells and naïve unmanipulated F1 animals. Data represented as mean \pm SEM. One way ANOVA with Tukey posttest was used for statistical analysis. 


\section{Long term SA-FasL-engineered donor cell recipients are functionally immune competent.}

One of the important aspects in HSCT recipients is the immune competency. The recipients should be competent enough to initiate immune responses against third party antigens, to ward off infections, while maintaining tolerance to allo-antigens. To assess the functional immunity in long term SA-FasL-engineered grafts recipients ( $>90$ days), we tested their ability to reject third party allografts. Two heterotopic skin grafts were applied to trunk of each mouse from TBI + BM group or SA-FasL-group (10 ng). One from $\mathrm{BALB} / \mathrm{c}\left(\mathrm{H} 2 \mathrm{~K}^{\mathrm{d}}\right)$, which is targeting alloantigen in GVHD settings, and one from $\mathrm{C} 3 \mathrm{H} / \mathrm{HeJ}$ third party $\left(\mathrm{H} 2 \mathrm{~K}^{\mathrm{K}}\right)$ donors (Fig. 12). Interestingly, all long-term animals transplanted with BALB/c skin grafts accepted the graft, indicating the presence of systemic tolerance against recipient alloantigens (Fig. 12A and B). In marked contrast, all third party $\mathrm{C} 3 \mathrm{H} / \mathrm{HeJ}$ skin grafts were rejected in both groups (Fig. 12A) with median survival of 22 days. These results suggest that, at this time after transplantation, $\mathrm{T}$ cell immune reconstitution was competent to enable rejection response against third party skin grafts, while maintaining tolerance to recipient allogenic antigens. 
A

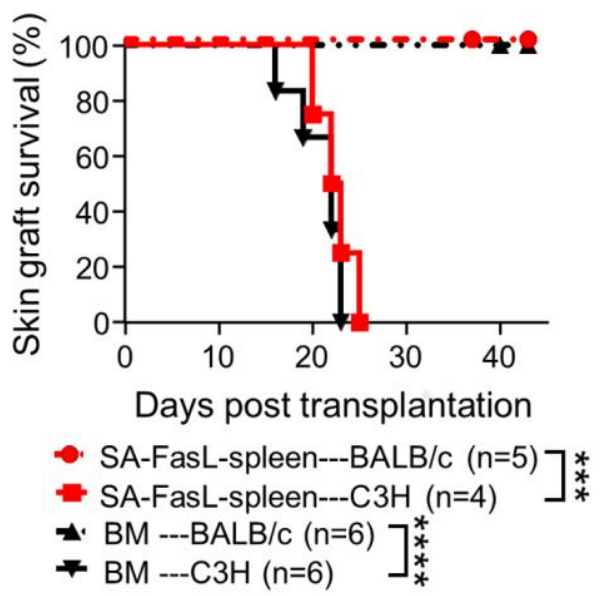

Day 7
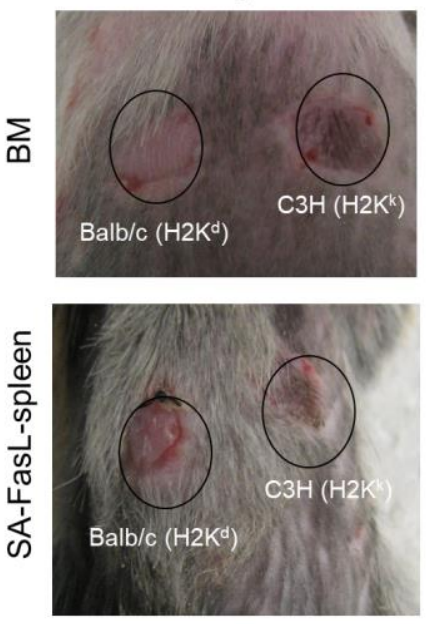

Day 35
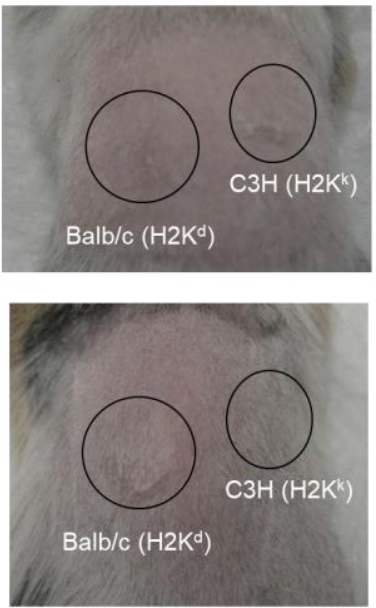

Figure 12. Long term SA-FasL-spleen recipients are immune competent. Long term animals that received bone marrow cells only without GVHD causing spleen cells and bone marrow cells along with SA-FasL-spleen cells were challenged with simultaneous donor $\left(\mathrm{BALB} / \mathrm{c}, \mathrm{H} 2^{\mathrm{b}}\right)$ and third party $\left(\mathrm{C} 3 \mathrm{H}, \mathrm{H} 2^{\mathrm{k}}\right)$ skin grafts. Animals were followed for complete graft rejection and noted as day of graft survival. (A) Skin allograft survival. (B) Representative pictures of skin grafts on day 7 and 35 post-transplantation. For comparison of survival curve log-rank (Mantel-cox) test was used. $* * * p<0.001, * * * * p$ $<0.0001$ 


\section{SA-FasL recipients exhibit reduced activated alloreactive Th1 phenotype.}

Although our data provide direct evidence that physical elimination of alloreactive $\mathrm{T}$ cells is an important mechanism of the observed prevention of GVHD in our model, apoptosis initiate by SA-FasL may set in motion other immunoregulatory mechanisms that may accentuate the efficacy of SA-FasL in our model. Thus we analyzed liver, mesenteric lymph nodes (mLN) by flow cytometry and target organs (colon, ileum and liver) by qRTPCR during the efferent phase of acute GVHD, day 21, the time point when control group animals that received unmodified spleen cells had significant loss in body weight. Activated $\mathrm{CD} 4^{+} \mathrm{T}$ cells $\left(\mathrm{CD} 4^{+} \mathrm{FoxP} 3{ }^{-} \mathrm{CD} 44^{+} \mathrm{CD} 62 \mathrm{~L}^{-} \mathrm{PD} 1^{+}\right)$and $\mathrm{CD} 8^{+} \mathrm{T}$ cells $\left(\mathrm{CD} 8^{+} \mathrm{CD} 25^{+}\right.$ and $\mathrm{CD} 8^{+} \mathrm{CD} 44^{+} \mathrm{CD} 62 \mathrm{~L}^{-} \mathrm{PD} 1^{+}$) were significantly higher in frequency in $\mathrm{mLN}$ and liver in the control group as compared with the SA-FasL group (Fig. 13A). Similar level of significance was also observed when analyzed as absolute numbers per gram in liver, but not in mLN (Fig. 14B). Interestingly, recipients of unmodified spleen cells had substantially higher frequency and absolute numbers of Treg cells $\left(\mathrm{CD} 4^{+} \mathrm{CD} 25^{+} \mathrm{FoxP} 3^{+}\right)$ than SA-FasL-spleen recipients in the liver and frequency, but not absolute cell numbers, in $\mathrm{mLN}$ (Fig. 13A and 14B). However, the Treg $\left(\mathrm{CD} 4^{+} \mathrm{CD} 25^{+} \mathrm{FoxP} 3^{+}\right)$and activated $\mathrm{T}$ effector $\left(\mathrm{CD} 4^{+}{ }^{\mathrm{F}}\right.$ oxP3 ${ }^{-} \mathrm{CD} 44^{+} \mathrm{CD} 62 \mathrm{~L}^{-} \mathrm{PD} 1^{+}$or $\left.\mathrm{CD} 8^{+} \mathrm{CD} 44^{+} \mathrm{CD} 62 \mathrm{~L}^{-} \mathrm{PD} 1^{+}\right)$cell ratio was not significant between the control and SA-FasL groups in the tissues analyzed (Fig. 13B). These observations suggest that the primary mechanism that SA-FasL prevents acute GVHD is physical elimination of alloreactive $\mathrm{T}$ effector cells, resulting in reduced frequency and absolute numbers in the target tissues.

qRT-PCR analysis of GVHD target tissues (liver, colon and small intestine) from

control group that received unmodified spleen cells had significantly higher expression 
profile of inflammatory cytokines (IFN- $\gamma$, TNF- $\alpha$, and IL-6), all are known mediators of acute $\mathrm{GVHD}^{154}$, as compared with SA-FasL-spleen recipients (Fig. 13C). In addition, GVHD control group also had higher level of T-bet transcription factor, a master regulator of Th1 differentiation ${ }^{155}$, than SA-FasL-spleen recipients in all GVHD target organs (Fig. 13C). Interestingly, upregulated Th1 response in GVHD control group was associated with augmented expression level of IL-4, mediator of Th2 differentiation, in liver $(p=0.0084)$ and colon $(\mathrm{p}=0.0205)$ of SA-FasL-spleen recipients as compared with GVHD control group without an apparent difference in small intestine (Fig. 13C). Similarly, the levels of GATA3, a master regulator of Th2 differentiation ${ }^{156}$, transcript was significantly higher ( $\mathrm{p}<0.0001)$ in large intestine, but not the liver or small intestine, of SA-FasL-spleen recipients than GVHD controls (Fig. 15). GVHD control group also had higher level of other inflammatory mediators, including IL-1 $\beta(p=0.0111)$, but the level of IL23p40 did not reach statistical significance $(\mathrm{p}=0.1006)$ in the liver (Fig. 15). We observed a similar pattern in the large intestine, but in the small intestine SA-FasL-spleen recipients had significantly higher expression level of IL23p40 ( $p=0.0001$; Fig. 15). SA-FasL-spleen recipients had significantly less transcripts of chemokine CCL2 in liver $(p=0.0099)$ and in small intestine $(\mathrm{p}=0.0172$; Fig. 13). Interestingly, SA-FasL-spleen recipients had significantly higher expression level of ROR $\gamma$ t in the liver $(\mathrm{p}=0.0003)$ and small intestine $(p=0.0004)$, but significantly lower expression in large intestine ( $p<0.0001$; Fig. 15). In marked contrast, when analyzed for FoxP3, transcription factor for Treg cells, GVHD control animals had higher level than SA-FasL-spleen recipients (Fig. 15).

The qRT-PCR data was further corroborated by intracellular cytokine assay using spleen cells stimulated with phorbol myristate acetate (PMA) and ionomycin. SA-FasL- 
spleen cells recipient had significantly lower frequency, but not absolute numbers, of CD4 ${ }^{+}$ cells secreting IFN- $\gamma(\mathrm{p}=0.0175)$ and TNF- $\alpha(\mathrm{p}=0.0125)$ (Fig. 16). We did not observe any difference between the groups for IFN- $\gamma$ or TNF- $\alpha$ or granzyme B producing $\mathrm{CD} 8^{+} \mathrm{T}$ cells (Fig. 16). Histologically, there was evidence of villous blunting (colon and ileum), intrahepatic infiltration and bile duct epithelial infiltration in the liver of GVHD control groups when compared with SA-FasL-spleen and BM recipients (Fig. 17). Taken together, these data suggest that SA-FasL-spleen recipients are protected from lethal acute GVHD by elimination of alloreactive $\mathrm{T}$ cells resulting in significantly reduced activated alloreactive $\mathrm{T}$ cells in the target organs by day 21 . This was further supported by significantly higher transcripts of proinflammatory cytokines, Th1 skewed $\mathrm{T}$ cell phenotype, and other inflammatory mediators in GVHD control animals that received unmodified spleen cells but not SA-FasL-spleen recipients. 


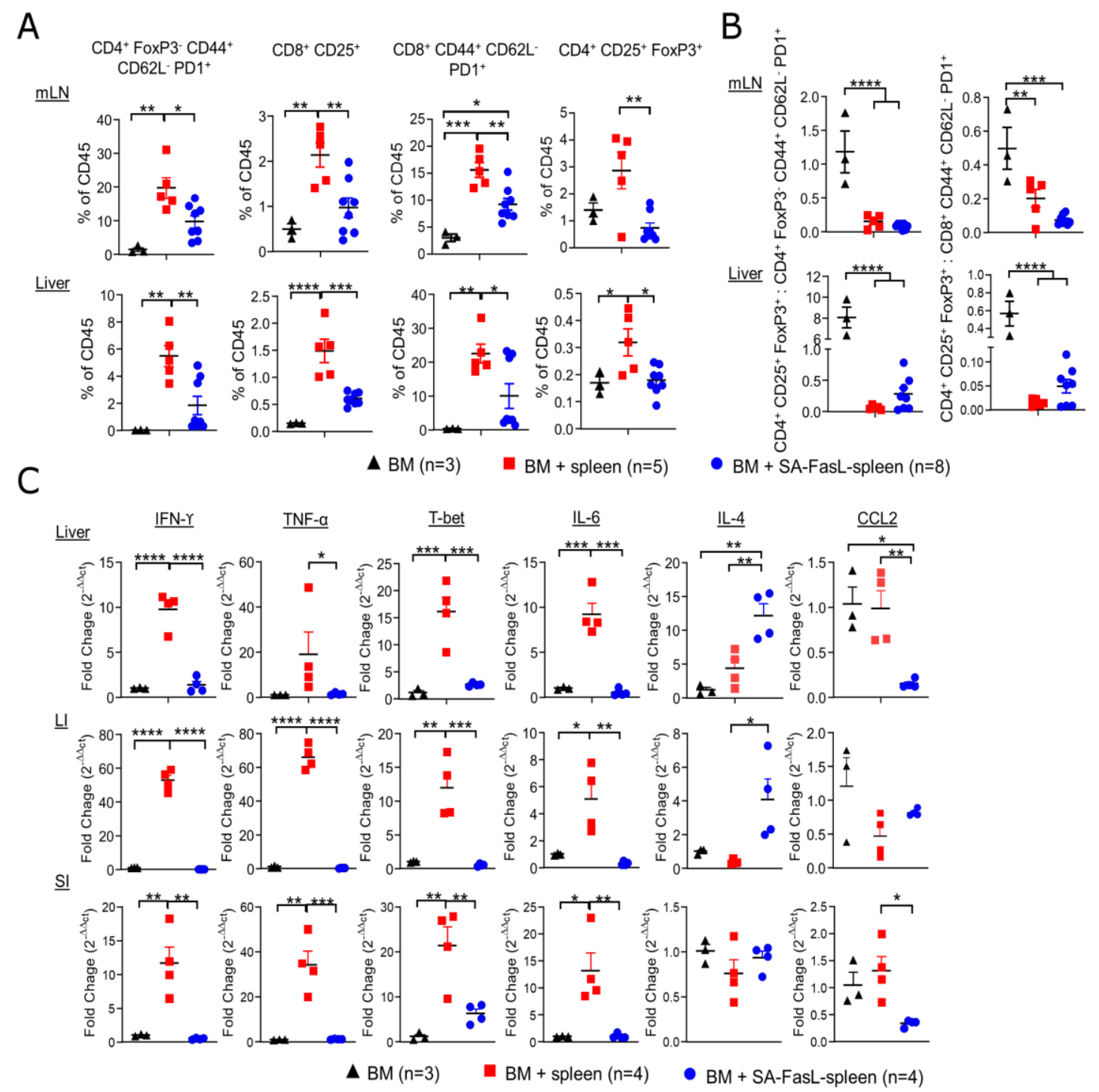

Figure 13. SA-FasL-spleen recipients have less activated cells and inflammatory mediators of acute GVHD at day 21 post transplantation. (A) Frequency of activated cells and regulatory $\mathrm{T}$ cells in mesenteric lymph nodes $(\mathrm{mLN})$ and the liver. Intrahepatic immune cells and mesenteric lymph nodes were analyzed for activated CD4 (CD4 ${ }^{+} \mathrm{FoxP} 3^{-}$ $\left.\mathrm{CD} 44^{+} \mathrm{CD} 62 \mathrm{~L}^{-} \mathrm{PD} 1^{+}\right)$and $\mathrm{CD} 8^{+} \mathrm{T}$ cells $\left(\mathrm{CD} 8^{+} \mathrm{CD} 25^{+} ; \mathrm{CD} 8^{+} \mathrm{CD} 44^{+} \mathrm{CD} 62 \mathrm{~L}^{-} \mathrm{PD} 1^{+}\right)$and Treg cells $\left(\mathrm{CD} 4^{+} \mathrm{CD} 25^{+} \mathrm{FoxP}^{+}\right)$. (B) Ratio of Treg cells to activated $\mathrm{CD}^{+}$and $\mathrm{CD} 8^{+} \mathrm{T}$ cells. (C) qRT-PCR analysis on target tissues [liver, large intestine (LI) and small intestine (SI)] 
at day 21 post transplantation. Total RNA from liver, colon and ileum was isolated and subjected to TaqMan based qRT-PCR assay for indicated cytokines, transcription factors, and chemokines. Fold change expression $\left(2^{-\Delta \Delta C t}\right)$ was calculated with respect to GAPDH as house-keeping gene and bone marrow only recipients. Data representative of two independent experiments and shown as mean \pm SEM. For comparisons, One way ANOVA with Tukey posttest was used in (A), (B) and (C). *p $<0.05^{* *} p<0.01, * * * p<0.001, * * * * p$ $<0.0001$ 


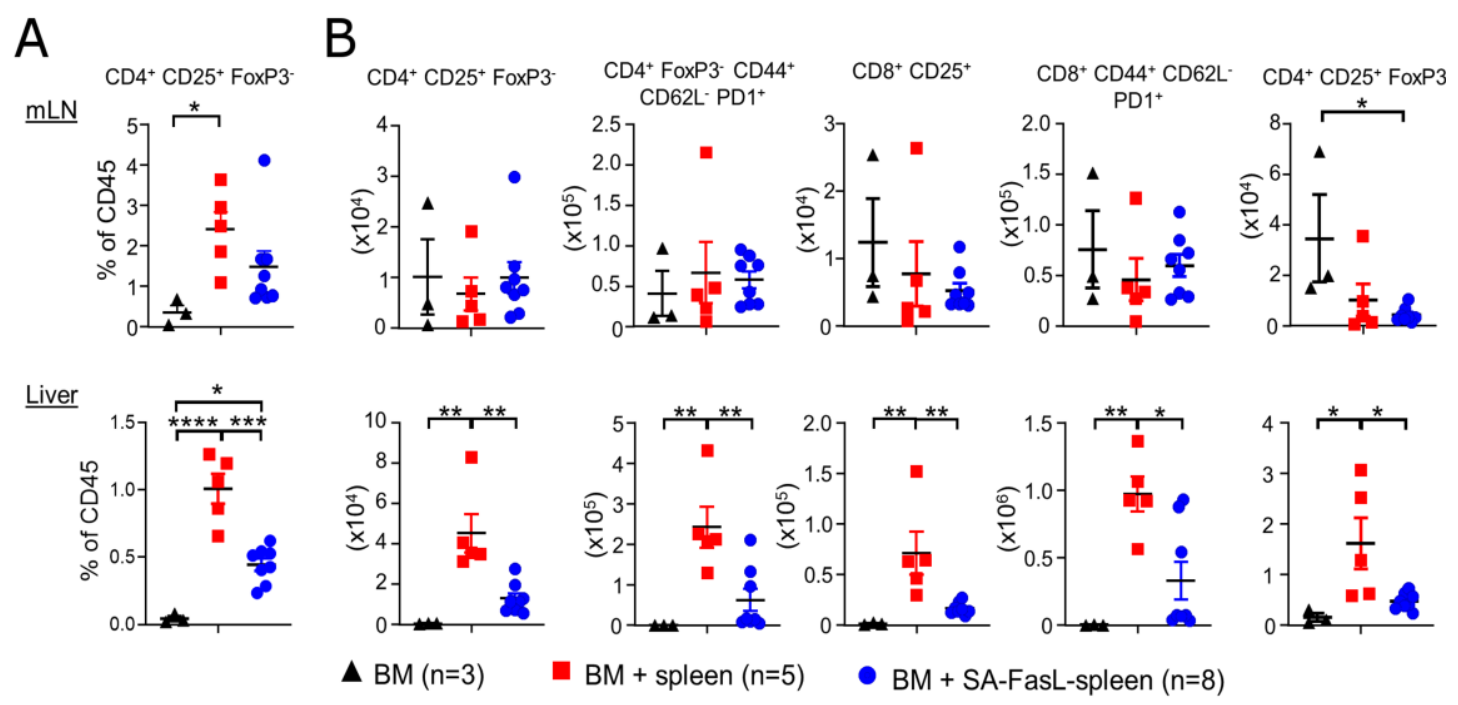

Figure 14. Immunophenotyping of recipients at day 21 post transplantation.

Mesenteric lymph nodes ( $\mathrm{mLN})$ and liver infiltrating cells were analyzed at day 21 posttransplantation. (A) Frequency of $\mathrm{CD}^{+}$activated $\mathrm{T}$ cells $\left(\mathrm{CD} 4^{+} \mathrm{CD} 25^{+} \mathrm{FoxP} 3^{-}\right)$. (B) Absolute number of $\mathrm{CD}^{+}$and $\mathrm{CD}^{+}$activated T cells and Treg cells in $\mathrm{mLN}$ and liver. Data pooled from two independent experiments and shown as mean \pm SEM. For comparison, One way ANOVA with Tukey posttest was used in (A) and (B). *p $<0.05 * * p<0.01, * * * p<0.001, * * * * p<0.0001$ 


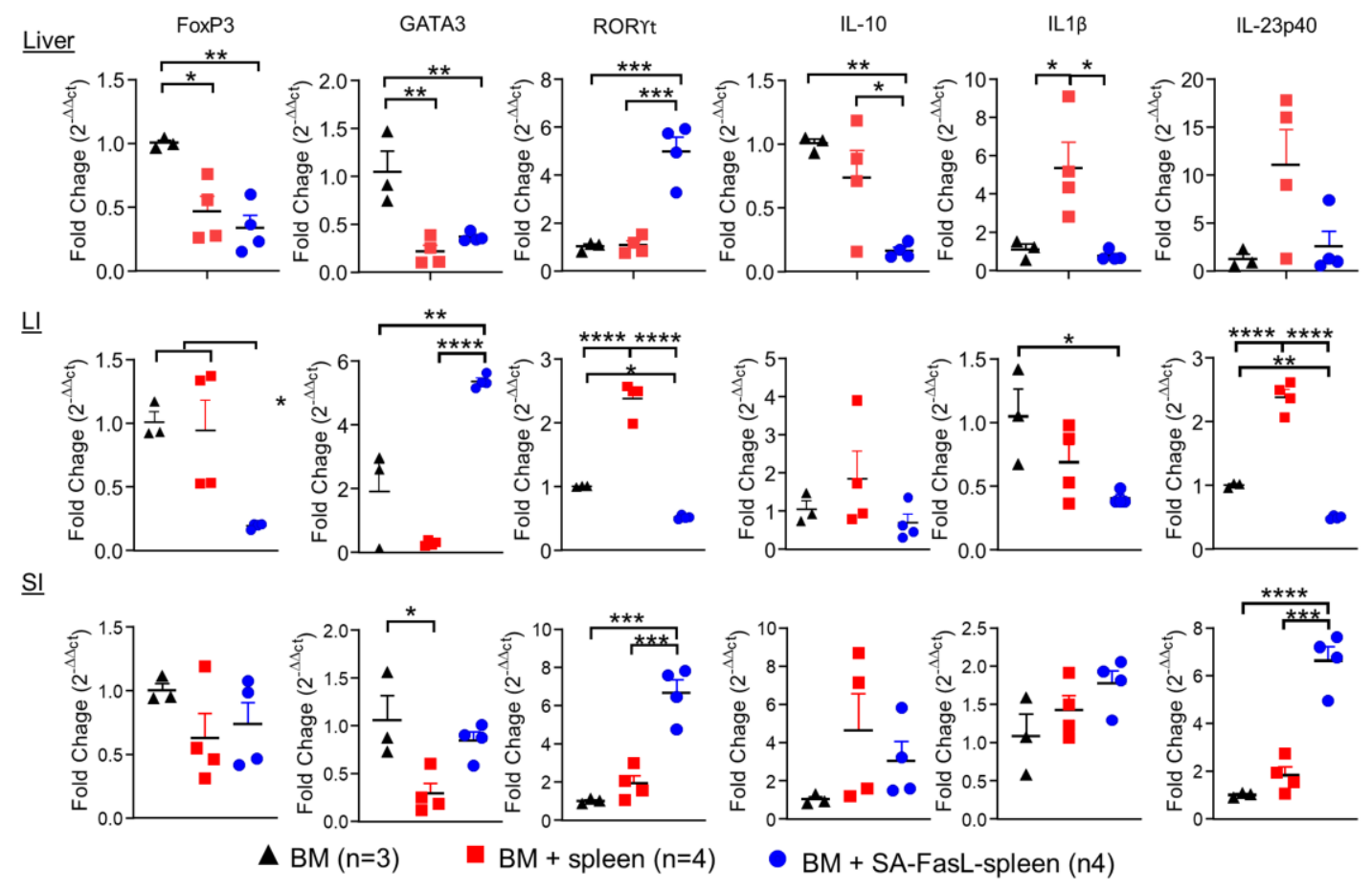

Figure 15. qRT-PCR analysis of GVHD target organs for immune markers. Total RNA from liver, colon, and ileum was isolated and subjected to TaqMan based qRT-PCR for the expression profile of multiple immune genes as indicated. Fold expression $\left(2^{-\Delta \Delta C t}\right)$ was calculated with respect to GAPDH, as a house keeping gene, and bone marrow only recipients. Data represented as mean \pm SEM. One way ANOVA with Tukey posttest was used for statistical comparison. $* \mathrm{p}<0.05 * * p<0.01, * * * p<0.001, * * * * \mathrm{p}<0.0001$ 

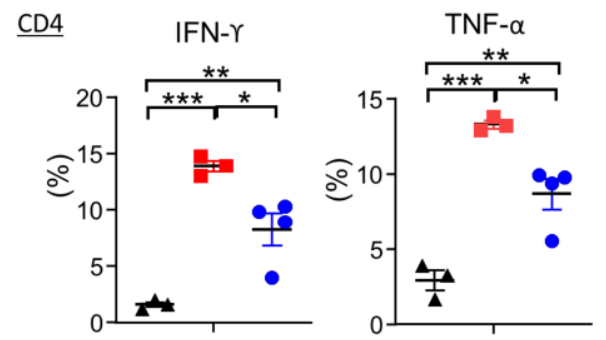

Granzyme-B
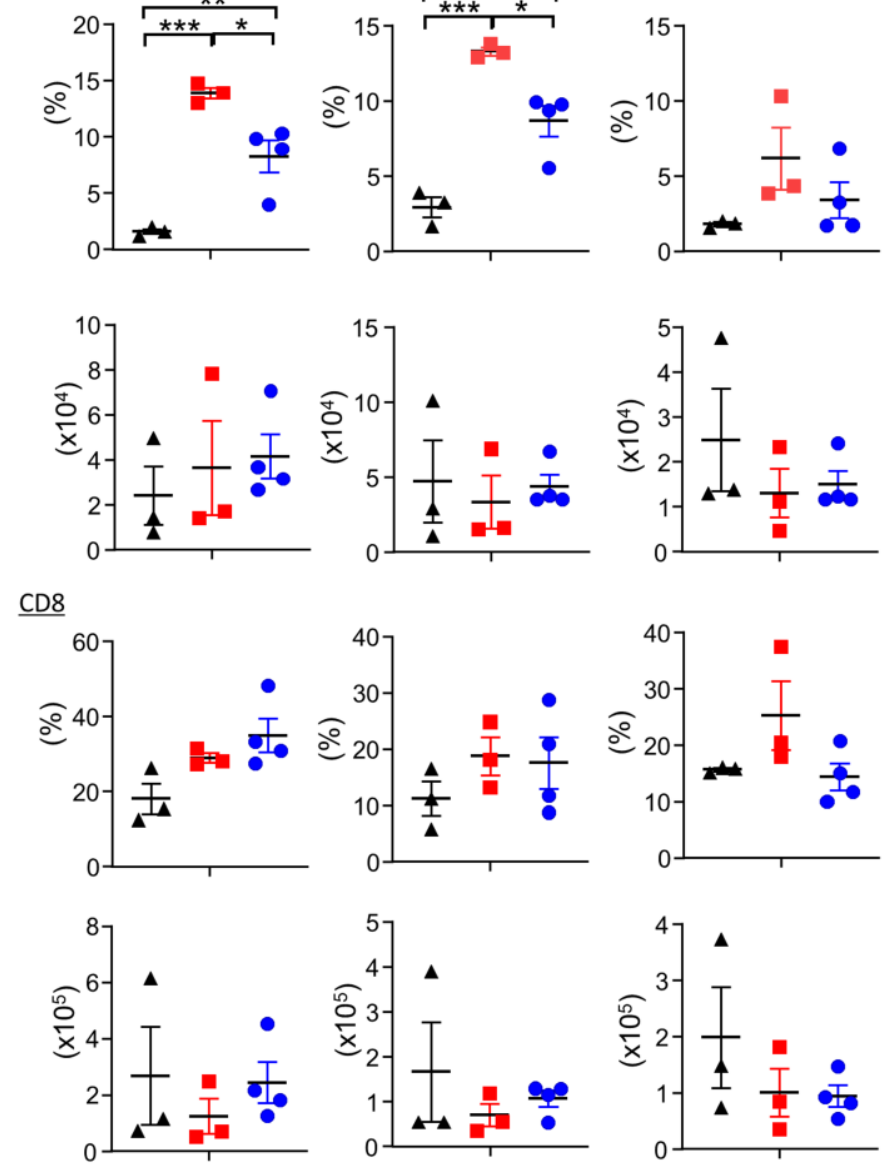

- $B M(n=3)$

- BM + Spleen $(n=3)$

- $B M+$ SA-FasL-Spleen $(n=4)$

Figure 16. Intracellular cytokine analysis on spleen cells. Frequency and absolute number of $\mathrm{CD}^{+}$and $\mathrm{CD}^{+} \mathrm{T}$ cells expressing IFN- $\gamma, \mathrm{TNF}-\alpha$, and granzyme B. After 21 days post transplantation, spleen cells were harvested from the indicated groups, stimulated with PMA and ionomycin, and analyzed using flow cytometry. Data are from cells pooled from multiple animals and represent two independent experiments. Data are represented as mean \pm SEM. One way ANOVA with Tukey posttest was used for statistical comparison. $* \mathrm{p}<0.05 * * p<0.01, * * * p<0.001, * * * * \mathrm{p}<0.0001$ 

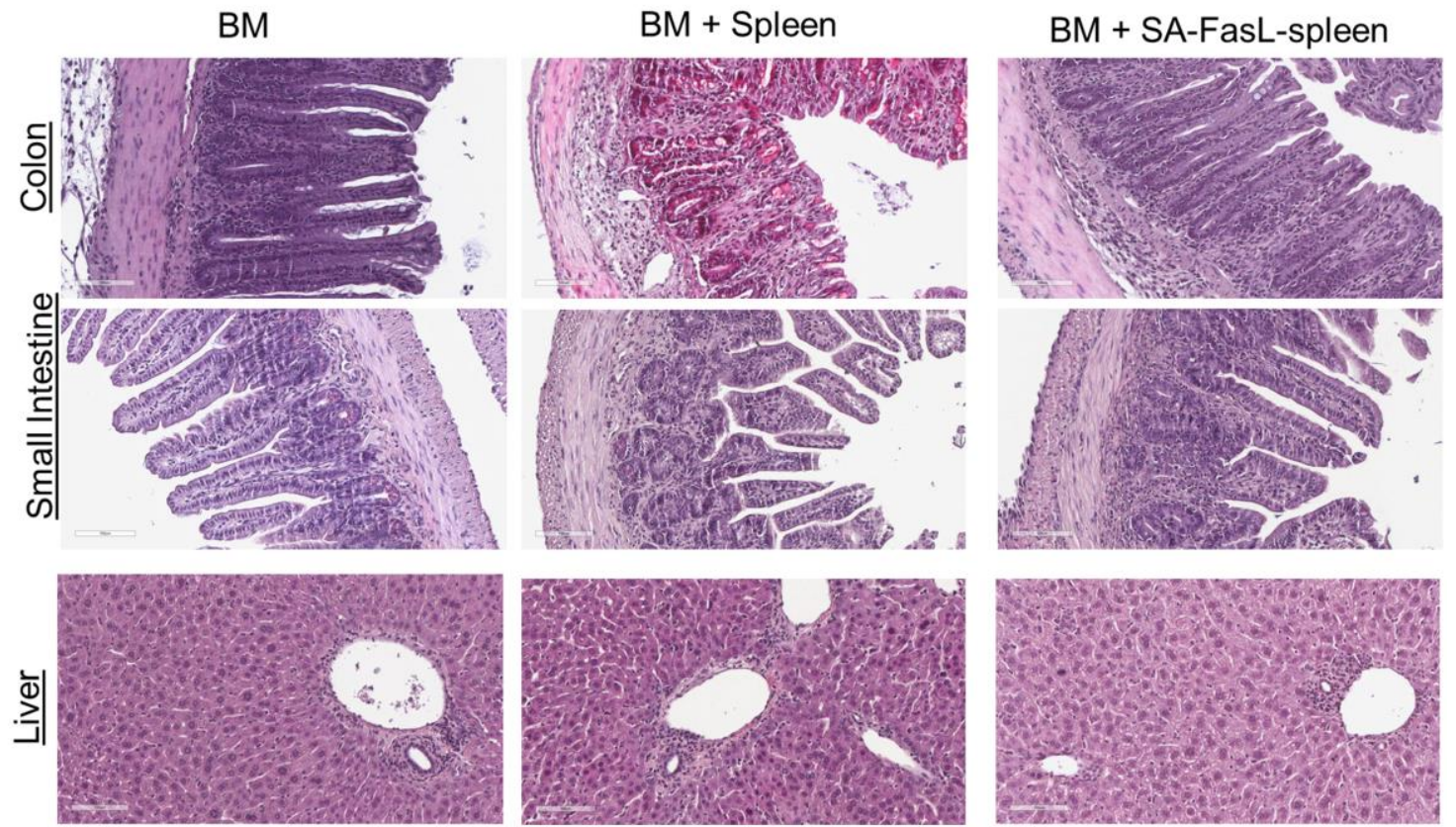

Figure 17. Representative H\&E staining of GVHD target organs from each cohort.

GVHD tissues were harvested at day 21 post transplantation, formalin fixed, and paraffin embedded. H\&E staining was performed on tissue section for the indicated groups. 


\section{Donor $\mathrm{CD25}^{+}$Treg cells are indispensable for the prevention of acute GVHD.}

$\mathrm{CD} 4^{+} \mathrm{CD} 25^{+}$regulatory $\mathrm{T}$ cells can suppress expansion of alloreactive $\mathrm{T}$ cells and inhibit lethal acute GVHD ${ }^{157,158}$. However, massive infusion of Treg cells at 1:1 ratio with $\mathrm{T}$ effector cells $\left(\mathrm{CD} 25^{-}\right)$is required for protection ${ }^{53,158}$. To assess the contribution of Treg cells for the protection against acute GVHD in our model, we depleted $\mathrm{CD} 25^{+} \mathrm{T}$ regulatory cell population from donor cell inoculum (Fig. 18) and then used the Treg-deplete splenocytes for adoptive transfer into lethally irradiated F1 recipients. Recipients transplanted with whole unmodified splenocytes developed fatal signs of acute GVHD with median survival time of 34 days. Whereas, animals receiving whole SA-FasL engineered splenocytes were protected from lethal GVHD with $80 \%$ of animals survived long term (>

60 days). Surprisingly, all F1 recipients of Treg-deplete splenocytes engineered with SAFasL developed signs of acute GVHD with severe diarrhea, hunched posture and decreased motion, and expired with a median survival time of 28 days as compared with 34 days for GVHD controls (Fig. 19; $\mathrm{p}=0.043$ ). Taken together, these results demonstrate the critical role of donor Treg cells in the prevention of acute GVHD in our model. 


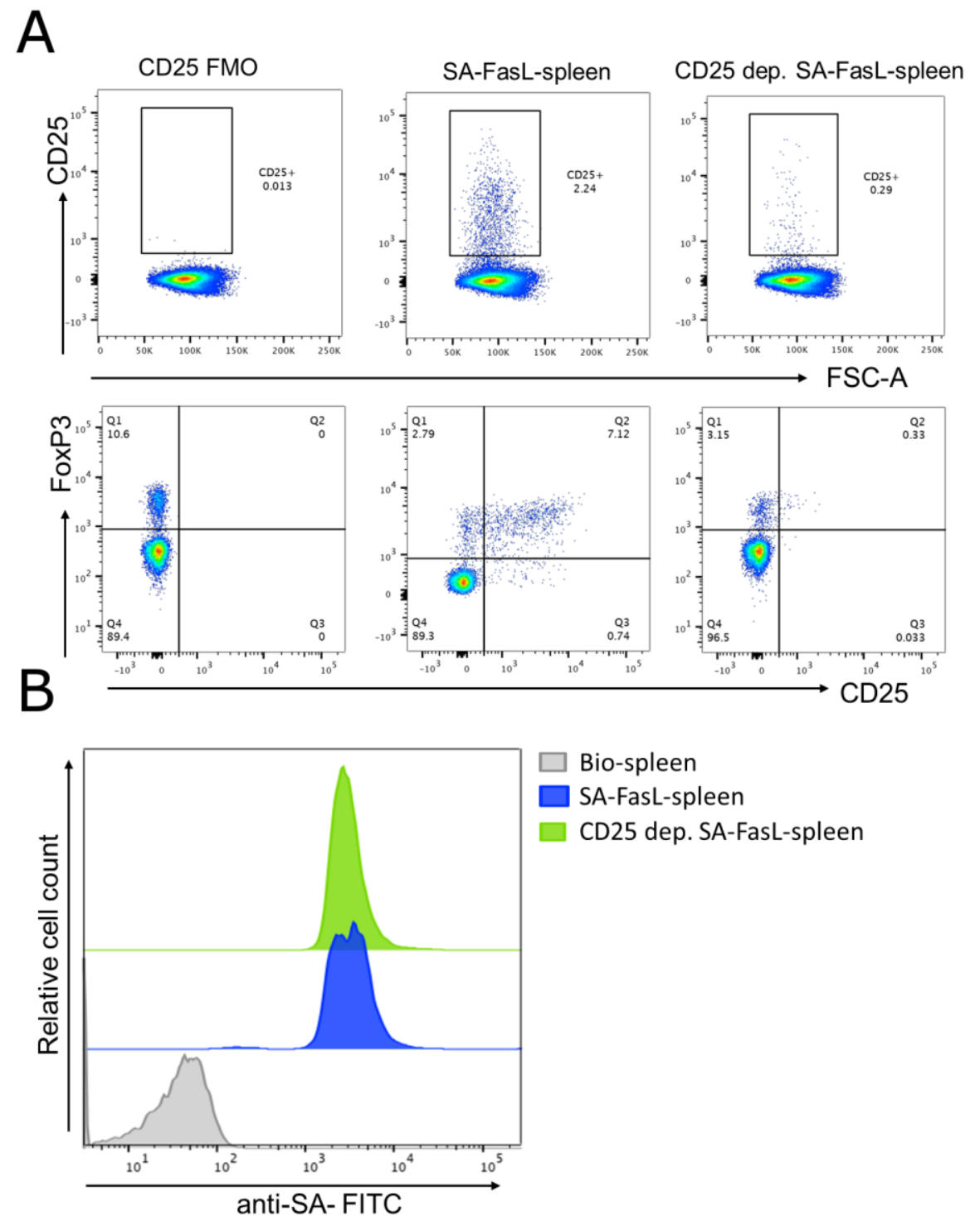

Figure 18. Depletion of $\mathrm{CD25}^{+}$cells from donor inoculum. (A) Representative flow dot plot for $\mathrm{CD} 25^{+}$and FoxP3 ${ }^{+} \mathrm{CD} 25^{+}$cells in SA-FasL-spleen and CD25 depleted SAFasL-spleen inoculum, indicating significant depletion of $\mathrm{CD} 25^{+}$cells. (B) SA-FasL engineering level on CD25+ cells depleted inoculum. Histogram plot suggesting similar level of SA-FasL engineering on $\mathrm{CD} 25^{+}$replete inoculum and $\mathrm{CD} 25^{+}$deplete inoculum. 


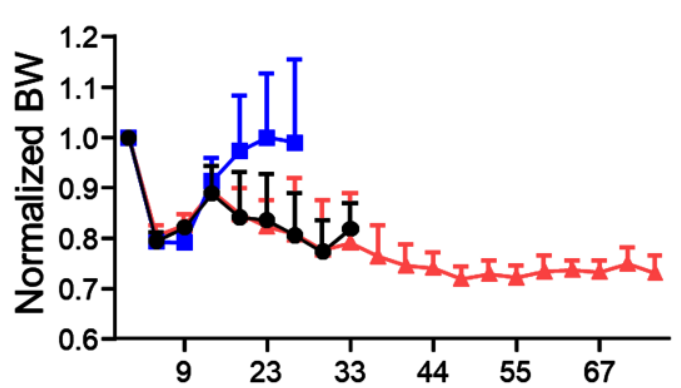

Days post transplantation
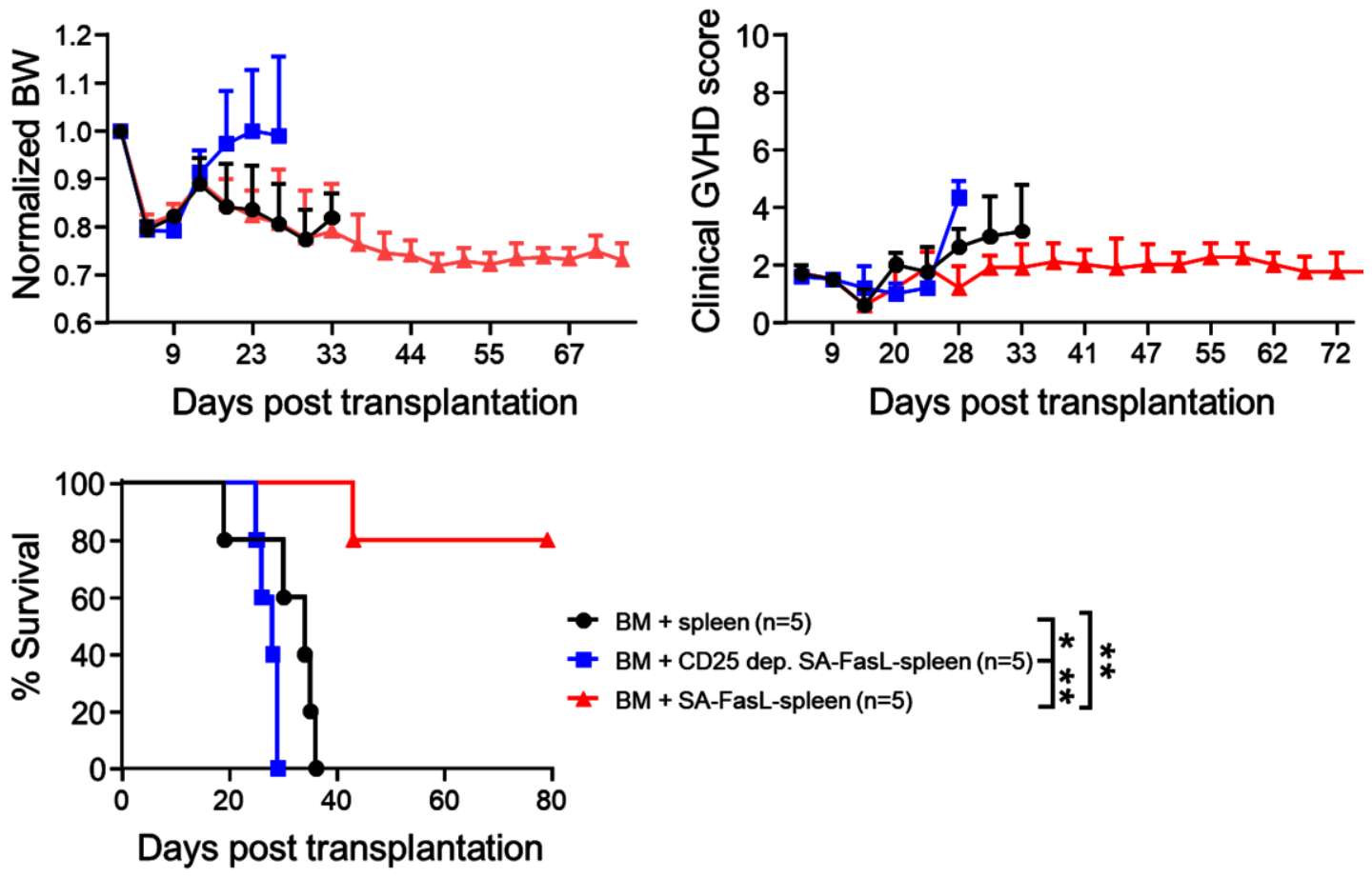

Figure 19. $\mathrm{CD}^{+}{ }^{+}$cells are indispensable for observed protective effect of SA-FasL for acute GVHD. Lethally irradiated F1 mice received bone marrow cells admixed with unmodified or SA-FasL engineered spleen cells or SA-FasL engineered CD25-deplete spleen cells. Animals were monitored for signs of acute GVHD and survival. For survival curve comparison, log-rank (Mantel-Cox) test was used. ${ }^{*} \mathrm{p}<0.05^{* *} p<0.01$ 


\section{SA-FasL engineering of human PBMCs abrogates acute GVHD in a humanized mouse model.}

To extend the observed effect of SA-FasL engineering on human immune response, we first performed in vivo tracking of human cells using NOD-scid-IL2 $\gamma \mathrm{R}^{\text {null }}$ (NSG) model. Neutrophil depleted human PBMC were engineered with SA-FasL or SA (control protein). NSG recipients were irradiated at $200 \mathrm{cGy}$ followed by $i . v$ injection of $5 \times 10^{6}$ SA- or SAFasL engineered human PBMCs. When analyzed 5 days post infusion, spleen of SArecipients had significantly greater number (Fig. 21A) as well as frequency (Fig. 20) of total human cells and human $\mathrm{T}$ cells as compared with SA-FasL group. Importantly, we observed a similar pattern in the liver (Fig. 21A and 20), a major target in human PBMC induced xenogeneic GVHD. Following this observation, we established a model of acute xenogeneic GVHD by transferring human PBMC, adapted from King et al. ${ }^{148}$. When 200 cGy preconditioned NSG animals were infused with $10 \times 10^{6}$ human PBMC, animals developed clinical signs of GVHD by day 14, including significant weight loss, hunched posture, ruffled fur and reduced motion with median survival time of 15 days (Fig. 21B). Delivery of SA-engineered human PBMCs had similar trend of developing fatal GVHD with median survival time of 15 days (Fig. 21B). However, engineering the human PBMCs with SA-FasL before transfusion significantly extended the survival $(\mathrm{p}<0.0001)$ with $27.67 \%$ of animals surviving for $>60$ days (Fig. 21B). There was a significant delay in the onset of GVHD with significant body weight loss by day 25 as compared with day 13 for the SA group. Taken together, these data suggest that engineering alloreactive T cells with SA-FasL is an efficient strategy to eliminate pathogenic cells to mitigate fatal acute GVHD in a xenogeneic humanized NSG model. 

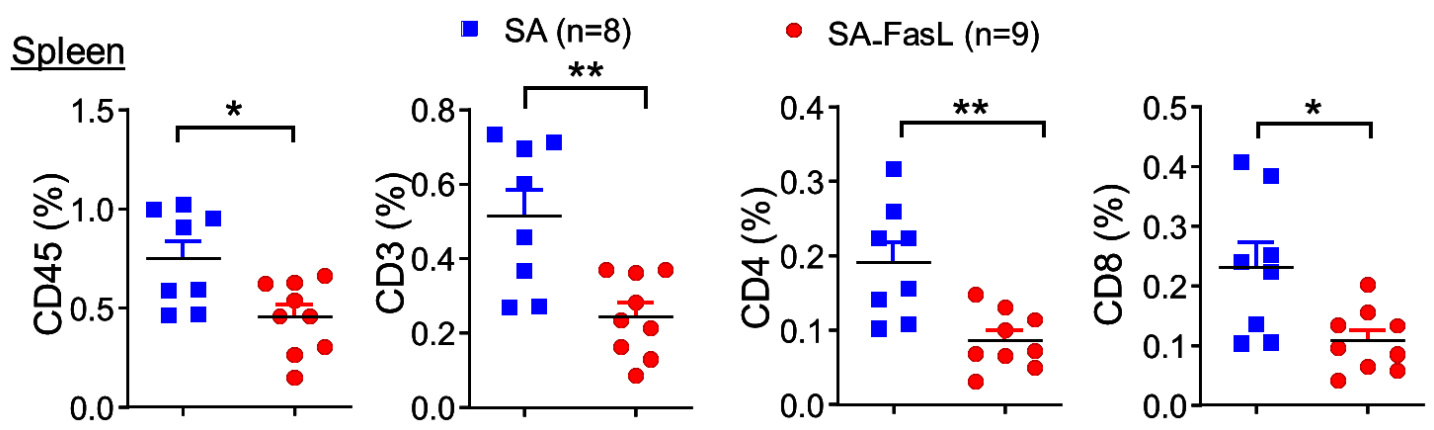

Liver
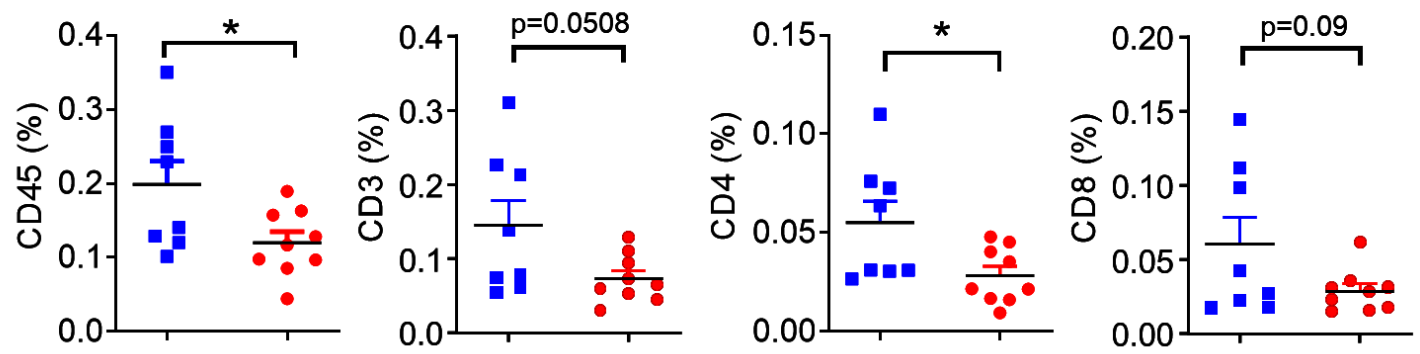

Figure 20. Frequency of human immune cells recovered from preconditioned NSG

animals. Preconditioned (200 cGy) NSG animals were transplanted with SA or SA-FasL engineered human PBMC. After 5 days post transplantation, spleen and liver of NSG recipients were analyzed for human immune cells by flow cytometry. For comparison of means, unpaired two tailed T- test was used. Data shown as mean \pm SEM. ${ }^{*} \mathrm{p}<0.05^{* *} p<$ 0.01 


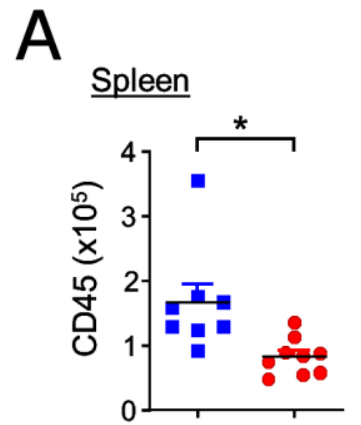

- $S A(n=8)$

- SA_FasL $(n=9)$

Liver
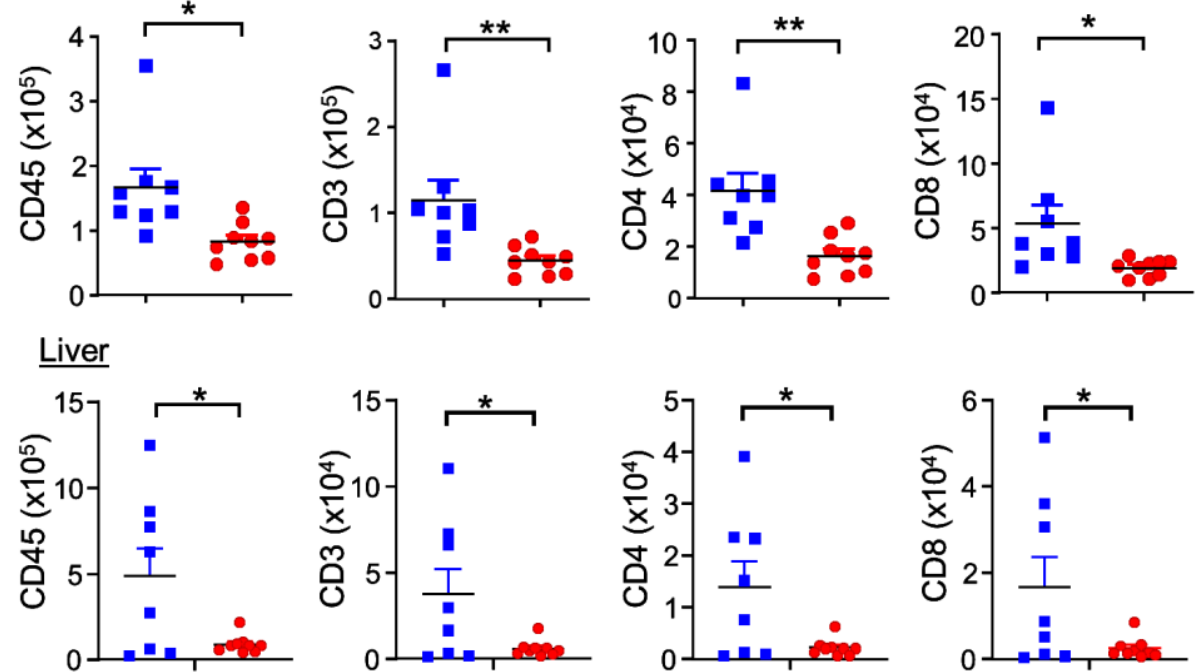

$B$
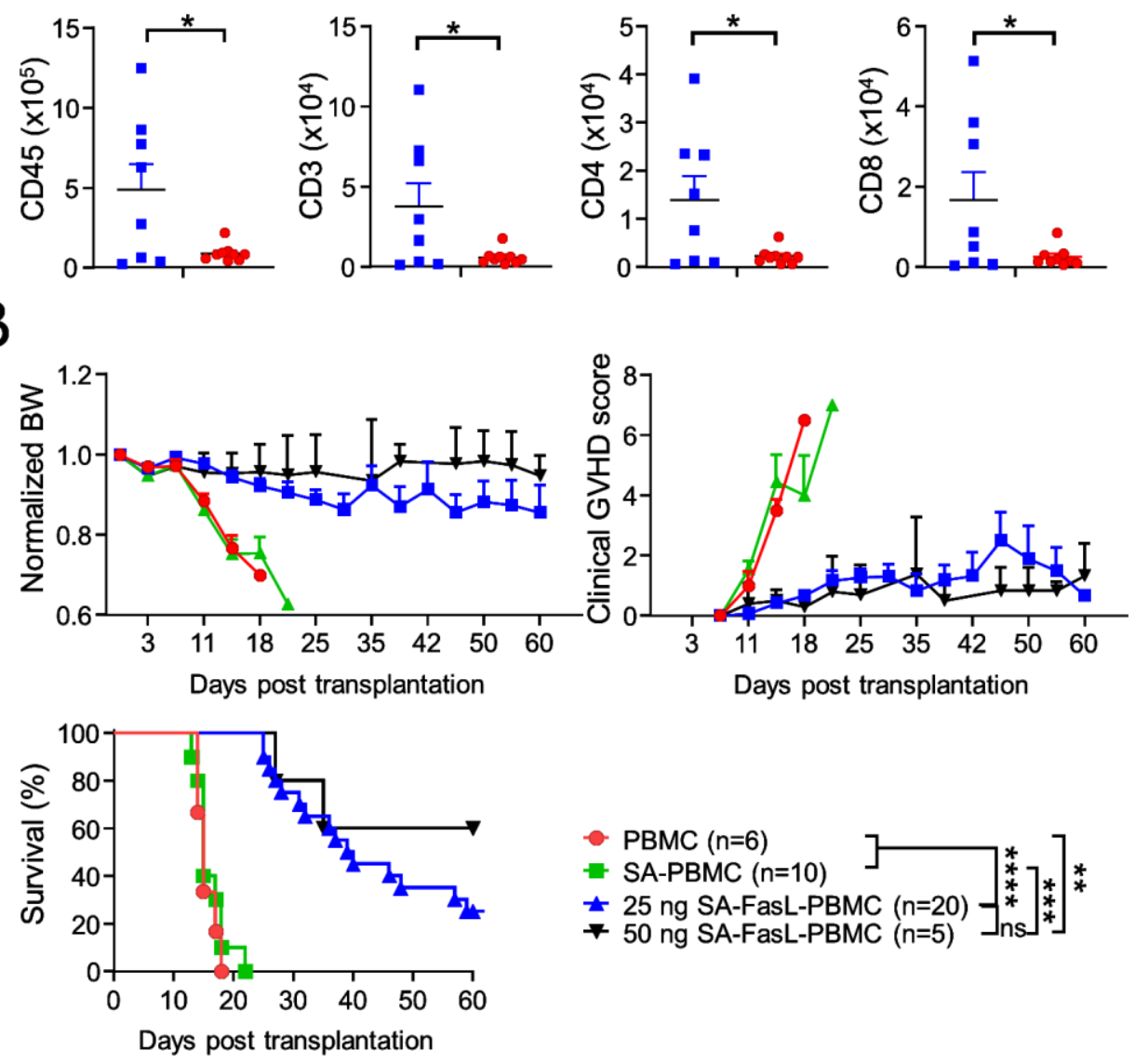

Figure 21. SA-FasL engineering eliminates activated human PBMCs and abrogates xenogeneic GVHD. (A) Absolute number of human cells recovered from preconditioned NSG animals. NSG animals were preconditioned at $200 \mathrm{cGy}$ and SA or SA-FasL 
engineered neutrophil depleted PBMCs were i.v injected. After 5 days post injection, spleen and liver were analyzed by flow cytometry. Data pooled from three independent experiments. (B) Xenogeneic GVHD is prevented from SA-FasL engineered PBMCs. Preconditioned NSG animals (200 cGy) were transplanted with unmodifed or SA/SA-FasL engineered human PBMC. Animals were followed for development of xenogeneic GVHD. For comparison of means, unpaired two tailed t-test was used in (A). For survival curve comparison log-rank (Mantel Cox) test was used. Data shown as mean \pm SEM. *p $<0.05^{* *}$, $p<0.01, * * * p<0.001, * * * * \mathrm{p}<0.0001$ 


\section{Discussion}

Despite various preventive strategies being implemented in the clinic for modulation of pathogenic function of alloreactive donor $\mathrm{T}$ cells, significant incidence of acute GVHD remains a challenge and limits the broad application of HSCT as a therapeutic modality for various disorders. Clinically, the major challenge is to reduce the severity of GVHD without simultaneously compromising the beneficial effects of $\mathrm{T}$ cell mediated immune responses. We herein describe a novel strategy to physically deplete alloreactive $\mathrm{T}$ cells in vivo as an effective means of preventing acute GVHD in a mouse model simulating HSCT in haploidentical clinical setting. We employed Fas/FasL mediated AICD as a strategy to specifically purge out alloreactive T effector cells to prevent acute GVH. AICD via Fas/FasL pathway is an important homeostasis mechanism to limit clonal expansion of activated $\mathrm{T}$ cells at the down phase of immune responses. AICD has been demonstrated in human and murine $\mathrm{T}$ cells following activation and plays a vital role in the maintenance of peripheral $\mathrm{T}$ cell tolerance ${ }^{35,36,128,159}$. Mature $\mathrm{T}$ cells acquire specific AICD susceptibility after prolonged activation and are dependent on repetitive TCR engagement with the antigen and cell cycle progression, thus spearing resting $\mathrm{T}$ cells $^{34,136,160}$. Using the ProtEx ${ }^{\mathrm{TM}}$ platform technology pioneered in our lab ${ }^{145}$, we engineered donor $\mathrm{T}$ cells to transiently display on their surface a novel from of the Fas death ligand, FasL, molecule chimeric with a modified form of streptavidin, SA-FasL. Our collective results show that this strategy efficiently depletes activated alloreactive T cells, resulting in the prevention of lethal acute GVHD in an allogeneic and a humanized mouse model. 
$\mathrm{T}$ cells were effectively engineered with SA-FasL and underwent apoptosis following activation by alloantigens both in vitro and in vivo. Coculture of SA-FasLengineered alloreactive $\mathrm{T}$ cells with unmodified cells in a mixed lymphocyte in vitro study demonstrated that apoptosis occurs primarily through an autocrine fashion by engagement of SA-FasL with Fas receptor on the same T cells. Importantly, SA-FasL was effective at femtogram levels in the elimination of alloreactive $\mathrm{T}$ effector cells in vivo. These observations are consistent with reports by others demonstrating that membranous, but not soluble, FasL is effective in the induction of apoptosis by crosslinking Fas receptor ${ }^{161}$. Our published and unpublished studies in solid organ and islet transplantation models ${ }^{129-131}$ using SA-FasL also corroborate the present findings. Interestingly, SA-FasL also eliminates donor Treg cells in our model. A study by Fritzsching et al. ${ }^{144}$ demonstrated that freshly isolated naive Treg cells $\left(\mathrm{CD} 4^{+} \mathrm{CD} 25^{+} \mathrm{FoxP} 3^{+}\right)$have higher susceptibility to Fas-mediated apoptosis post TCR restimulation confers resistance to Fas-mediated apoptosis in contrast to $\mathrm{T}$ effector cells. This suggest that SA-FasL on Treg cells provides apoptotic signals early on before this cell population being activated, whereas SA-FasL deliver apoptotic signals in $\mathrm{T}$ effector cells after TCR activation.

We then established a clinically relevant parent $\left(\mathrm{C} 57 \mathrm{BL} / 6, \mathrm{H}-2^{\mathrm{b}}\right)$ to $\mathrm{F} 1$ (C57BL/6xBALB/c, H-2 ${ }^{\mathrm{b} / \mathrm{d}}$ ) haploidentical HSCT model. In as much as transplantation of whole bone marrow cells into a lethally irradiated mouse does not develop GVHD, unlike rats and humans, we admixed bone marrow cells with autologous splenocytes and demonstrated the transplantation of this cell mixture from C57BL/6 into lethally irradiated F1 recipients resulted in acute GVHD. Using this model, we demonstrated that donor cells containing splenocytes engineered with SA-FasL was effective in preventing acute GVHD, 
whereas all mice receiving SA control protein engineered cells showed classical signs of GCHD, including hunched posture, decrease in mobility, ruffled fur and skin rashes along with diarrhea, and succumbed to acute GVHD and expired. As a prelude to clinical translation of this approach, we also demonstrated that human T cells engineered with SAFasL underwent apoptosis in response to mouse xenoantigens in vivo that resulted in significant protection against acute GVHD.

Alloreactive $\mathrm{T}$ cells mediated destruction of primary and secondary lymphoid organs is a good indicator of acute GVHD and is responsible for the lack of immune reconstitution following $\mathrm{HSCT}$ in conditioned recipients ${ }^{162}$. Inefficient immune reconstitution contributes to frequent opportunistic infections and relapse. When long term surviving animals (>100 days) were analyzed for immune composition, they were fully donor chimeric with efficient engraftment and better frequency of immune cell components as compared with long term surviving animals that received only bone marrow cells, but not GHVD causing spleen cells. Efficient immune competency was further confirmed by demonstrating that long term survivors rejected third party, but not those animals that received donor skin allografts. These findings also indicate that SA-FasL selectively purges out alloreactive $\mathrm{T}$ cells, leading to systemic tolerance to donor alloantigens, but preserves non-alloreactive $\mathrm{T}$ cells so that they generate an effective immune response to donor-unrelated antigens.

Clinical GVHD involves significant target tissue tropism that includes recognition of either major or minor histocompatibility antigens followed by proliferation in lymphoid organs and target tissue infiltration resulting in significant tissue damage. Multiple studies suggest that the GVHD progression is highly time sensitive. Allogeneic T cell activation 
occurs in several lymphoid organs at early time point (afferent phase day 6) followed by dramatic expansion of effector $\mathrm{T}$ cells ${ }^{163,164}$. Activation of alloreactive $\mathrm{T}$ cells occurs in draining lymph nodes rather than peripheral lymph nodes. Clinical significance is supported by the inability of memory T cells to induce lethal acute GVHD ${ }^{165}$, despite their ability to alloreactivity as naïve T cells. Furthermore, CD62L $\mathrm{L}^{+}$Treg, but not CD62 $\mathrm{L}^{-}$, cells are capable of suppressing acute GVHD in vivo, despite both cells being able to suppress alloreactive $\mathrm{T}$ cell proliferation in vitro ${ }^{166,167}$. Study by Nguyen et al. ${ }^{167}$ suggested that alloreactive $\mathrm{T}$ cells had similar pattern of early activation, proliferation and localization with respect to Treg cells. However, persistent BLI (bioluminescence imaging) signals indicated that proliferation of alloreactive $\mathrm{T}$ cells continued to increase in lymphoid tissues and target organs in parallel with development of signs of GVHD. In addition, they show that Treg cells as cellular therapy to prevent acute GVHD is time and dose dependent, such that earlier infusion of Treg cells led to greater reduction of effector $\mathrm{T}$ cells proliferation and better survival. The observed protective effect is dose dependent requiring higher doses of Treg cells to reverse established inflammation. In our study, SA-FasL engineering induced apoptosis of alloreactive $\mathrm{T}$ cells post infusion, thus significantly reducing their number and thereby limiting their proliferation. When analyzed at day 21 post transplantation (effector GVHD phase), SA-FasL-spleen cells recipient had significantly less activated $\mathrm{CD}^{+}$and $\mathrm{CD} 8^{+} \mathrm{T}$ cells, but these animals also had substantially less Treg cells. The Treg/T effector ratio is a critical determinant of suppression; however, SA-FasLspleen and unmanipulated spleen recipients had similar level of Treg/activated T effector ratios. However, the depletion of donor Treg cells before transplantation negated the 
beneficial effect of SA-FasL, providing direct evidence that Treg cells play a critical role in our model.

The source of discrepancy between the transplantation of Treg-depleted cells and phenotyping at day 21 post-transplantation in our model is unknown and may be due to several factors. First, FoxP3 as a canonical marker of Treg cells does not define their regulatory capacity. Treg cells show plasticity dictated by the nature of the microenvironment they function in. In particular, Treg cells can assume pathogenic functions in response to excessive inflammation. IFN- $\gamma$ has been shown to limit Treg cell function ${ }^{168}$ and acute GVHD is characterized by a highly inflammatory state. Expression of FoxP3, a master regulator of Treg cells, alone is not adequate for functional stability. The suppressive function of these cell population is well correlated with DNA methylation status of FoxP3 CNS2 region ${ }^{169}$. Acute GVHD has been considered a Th1-type disease dominated by cytotoxic $\mathrm{T}$ cell mediated pathology with increased production of Th1 cytokines. When analyzed by qRT-PCR, SA-FasL-spleen recipients had significantly less Th1 phenotype (T-bet, IFN- $\gamma$, TNF- $\alpha$ ) and other proinflammatory mediators (IL-6) than control groups. Instead, SA-FasL-spleen recipients had skewed differentiation with upregulated IL-4, GATA-3 and ROR $\gamma \mathrm{t}$. This is in line with previous reports demonstrating that the depletion of alloreactive Th1 $\mathrm{T}$ cells by AICD polarizes the residual $\mathrm{CD} 4^{+} \mathrm{T}$ cells to a Th2 phenotype, which may protect against acute GVHD $^{52,170}$. Treg cells has been implicated in therapeutic role to prevent acute GVHD. However, significant number of Treg cells needs to be infused and multiple experimental protocol for ex vivo expansion of donor Treg cells are under trial ${ }^{53,171}$. Interestingly, CD25 $5^{+}$Treg cells were still indispensable in the preventive strategy despite the significant elimination of alloreactive 
cells in vivo. This observation is similar to the high dose post-transplant cyclophosphamide strategy that requires Treg cells for its preventive effect ${ }^{172}$.

In conclusion, we demonstrate human and mouse $\mathrm{T}$ cells can efficiently be engineered with SA-FasL protein in a practical and clinically applicable manner and that this strategy is effective in preventing acute GVHD in two clinically relevant settings, myeloablative haploidentical and humanize mouse models. Engineering donor graft with SA-FasL provides efficient, attractive and facile means of modulating immune response to prevent lethal acute GVHD with significant translational potential. 


\section{CHAPTER 3}

\section{PANCREATIC ISLETS SURFACE ENGINEERED WITH CD47 INNATE IMMUNE CHECKPOINT SHOW ENHANCED ENGRAFTMENT FOLLOWING INTRAPORTAL ISLET TRANSPLANTATION}

\section{Introduction}

Islet transplantation is an important $\beta$-cell replacement therapy for refractory chronic pancreatitis and type 1 diabetes (T1D). A major limitation to clinical beta cell replacement therapy is a significant loss of islets immediate post-transplantation triggered by instant blood mediated inflammatory reaction (IBMIR) ${ }^{173,174}$. IBMIR is initiated when islets come into direct contact with the recipient blood following intraportal infusion and is responsible for the loss of $50-70 \%$ of the initial islet mass ${ }^{175,176}$. IBMIR is characterized by the activation of coagulation cascade, complement activation, and infiltration of myeloid cells that express various inflammatory mediators that result in the destruction of islets $^{80,84}$. Tissue factor (TF) and various other proinflammatory cytokines and chemokines, such as IL-1 $\beta$, IP-10, IL-6, CXCL8, CXCL10, CCL2, expressed by islets or resident antigen presenting cells trigger $\operatorname{IBMIR}^{79,84,88,177,178}$. As such, insulin independence rate for autologous islet transplantation at 5 years can be as low as $10 \%$ and multiple infusions of islets from different donors may be required to achieve insulin independence in recipients of allogeneic islet grafts ${ }^{179,180}$. Therefore, effective control of IBMIR will overcome an important limitation of clinical islet transplantation. 
CD47 is a ubiquitously expressed transmembrane glycoprotein of the immunoglobulin superfamily that serves as a ligand for the signal regulatory protein alpha $(\mathrm{SIRP} \alpha)$, an immune inhibitory receptor, expressed on the surface of myeloid cells ${ }^{119}$. The interaction of CD47 with $\operatorname{SIRP} \alpha$ serves an important innate immune checkpoint that delivers a "don't-eat-me" signal and acts as a critical regulator of "marker of self"119. The importance of $\mathrm{CD} 47 / \mathrm{SIRP} \alpha$ axis in self-recognition and inhibition of phagocytosis by macrophages was demonstrated by rapid clearance of red blood cells genetically modified to lack CD47 expression following infusion into CD47 competent syngeneic mice ${ }^{110}$.

$\mathrm{CD} 47-\operatorname{SIRP} \alpha$ pathway has been implicated in the regulation of both innate and adaptive immune responses. The system negatively regulates macrophage activation and phagocytosis $^{119}$, adhesion and activation of platelets and neutrophils ${ }^{116}$, attenuation of antibody-dependent cell-mediated cytotoxicity/phagocytosis (ADCC/ADCP) ${ }^{181,182}$, and adaptive immune responses ${ }^{183-185}$. These functional features of CD47 were shown to be extensively exploited for immune evasion by various tumors that highlighted the potential of CD47-SIRP $\alpha$ axis as an important target for cancer immunotherapy ${ }^{186}$. Indeed, various tumor cells were shown to have elevated levels of CD47 expression and its blockade using antibodies resulted in effective therapy in preclinical cancer models that led to current efforts to test this innate immune checkpoint blockade for cancer immunotherapy in the clinic $^{186-189}$

CD47/SIRP $\alpha$ pathway has been shown to play a critical role in innate allo- and xeno-recognition, independent of adaptive immunity ${ }^{190,191}$. Polymorphism in the SIRP $\alpha$ that alters the binding strength of CD47 is associated with rapid clearance of various cellular grafts, including hepatocytes ${ }^{120,185}$, insulinoma cells ${ }^{192}$, and hematopoietic stem 
cells ${ }^{108,193,194}$. Ectopic expression of recipient CD47 in porcine cells blocks their phagocytosis by human macrophages ${ }^{195}$. Similarly, hematopoietic cells from swine genetically modified to express human CD47 showed enhanced engraftment in a humanized mouse model of transplantation ${ }^{196}$.

Given the demonstrated role of CD47-SIRP $\alpha$ axis as innate immune checkpoint, we hypothesized that CD47 can be used to enhance syngeneic islet engraftment by mitigating IBMIR. We herein report the generation of a novel form of SA-CD47 molecule and demonstrate for the first time that that the transient display of CD47 protein on the surface of islets enhance engraftment and long-term function in a minimal mass intraportal islet transplantation mouse model by mitigating IBMIR. The rapid and transient display of SA-CD47 protein on the surface of tissues and cells to mitigate IBMIR provides a facile and clinically applicable platform with significant translational potential for transplantation of various tissue and cellular grafts.

\section{Materials and Methods}

\section{Animals}

C57BL/6 (CD45.2, H2K ${ }^{\mathrm{b}}$ ) mice were purchased from Jackson Laboratory. Animals were bred and maintained in our specific pathogen-free animal barrier facility at the University of Louisville, KY. All experiments were performed in accordance with the policies of the NIH for Guide for the Care and Use of Laboratory Animals and protocols approved by the University of Louisville Animal Care and Use Committee. 


\section{Construction, production, and physical characterization of SA-CD47 protein}

A synthetic gene encoding the extracellular domain of CD47 (19-161 bp; GI: AB012693.1) of C57BL/6 murine strain was subcloned C-terminus to a modified form of streptavidin in the drosophila pMT/BiP/V5-His $\mathrm{CuSO}_{4}$-inducible expression vector (Fig. 1) ${ }^{145}$. Drosophila S2 cells were transfected with the construct using the Celfectin II transfection system (Invitrogen) to establish stable transfectants. SA-CD47 expression was induced using $1 \mathrm{mM} \mathrm{CuSO}_{4}$, culture medium was collected 3 days later, and the protein was purified using a metal-ion charged sepharose column taking the advantage of the 6xHis tag engineered into the C-terminus (GE, Amersham). Purified protein was characterized using SDS-PAGE and Western blot analysis per published protocols ${ }^{130,145}$.

\section{Cell surface engineering with SA-CD47 protein}

Spleens from C57BL/6 or Lewis rat were harvested and processed into single-cell suspension using frosted slides. Red blood cells were lysed using a home-made buffered ammonium chloride solution. SA-CD47 engineering of spleen cells was done following previously reported protocol ${ }^{131}$. Cell surface was modified with biotin by incibation in 5 $\mu \mathrm{M}$ EZ-Link ${ }^{\mathrm{TM}}$ Sulfo-NHS-LC biotin (hereafter referred to as biotin) solution (Pierce) in sterile PBS at room temperature for 30 mins. Cells were then washed twice with sterile PBS followed by incubation with $200 \mathrm{ng}$ SA-CD47 or an equimolar amount of SA protein per $10^{6}$ cells at a final volume of $500 \mu \mathrm{l}$ of sterile PBS for 30 mins in a cold room by constant rocking. Cells were then washed and analyzed for the presence of biotin and SACD47 on the cell surface by staining with streptavidin-allophycocyanin (APC) and APClabelled anti-mouse CD47, respectively, using flow cytometry. 


\section{In vitro phagocytosis assay}

In vitro phagocytosis assay was done as described before ${ }^{112,183}$. Briefly, SA- and SA-CD47 engineered rat spleen cells were labeled with $2.5 \mu \mathrm{M}$ carboxyfluorescein succinimidyl ester (CFSE, Thermo Fisher Scientific) or $2.5 \mu \mathrm{M}$ Cell Trace Violet (CTV, Thermo Fisher Scientific), respectively, according to manufacturer's protocol and resuspended in complete RPMI (RPMI 1640 supplemented with 10\% FBS, Penicillin/ Streptomycin and 2 mM L-Glutamine). For single culture assay, SA-rat spleen cells or SA-CD47-rat spleen cells $\left(2.5 \times 10^{6} /\right.$ well) were cultured with mouse RAW 264.7 macrophage-like cell line $\left(0.5 \times 10^{6} /\right.$ well). For mixed culture settings, both fluorescence labeled target cells were mixed in a 1:1 ratio followed by co-culture $\left(2.5 \times 10^{6}\right.$ cells/well $)$ with RAW 264.7 cells $\left(0.5 \times 10^{6} /\right.$ well). Post incubation non-engulfed cells were washed, and adherent RAW cells were harvested and stained with anti-CD11b antibody prior to flow cytometric analysis. Phagocytosis assessed by measuring \% of double positive macrophages $\left(\mathrm{CD}_{11} \mathrm{~b}^{+} \mathrm{CFSE}^{+}\right.$ or $\left.\mathrm{CD}_{11 b^{+}} \mathrm{CTV}^{+}\right)$.

\section{Pancreatic islet isolation, engineering, and functional analysis}

Islet isolation was performed using Liberase ${ }^{\mathrm{TL}}$ enzyme (Roche) according to a standard protocol $^{128,130}$. Islets were engineered with the SA-CD47 or SA as a control protein by incubating first in $5 \mu \mathrm{M}$ biotin then with the indicated amount of protein for 30 mins $^{130,145}$. Alamar blue (AB, Thermo Fisher scientific) assay, that incorporates redox reagent that changes color when reduced in response to metabolic activity, was used to assess the effect of SA-CD47 engineering on viability and metabolic activity of islets ${ }^{197}$. Briefly, 100 
unmodified naïve or SA-CD47-engineered islets were cultured in complete RPMI media supplemented with $10 \% \mathrm{AB}$ reagent in a 96-well plate. After 6 hours of incubation, the reduced form of reagent soluble in the solution was measured as absorbance at a wavelength of $562 \mathrm{~nm}$ on SpectraMax microplate reader (Molecular Devices).

\section{Glucose-stimulated insulin secretion (GSIS) assay}

A static incubation protocol for GSIS assay was performed to assess whether engineering with SA-CD47 impacts islet function. Naïve islets or SA-CD47-engineered islets (100 islets per transwell) were incubated at $3 \mathrm{mM}$ glucose solution, prepared in Krebs ringer bicarbonate buffer, for 1 hour for equilibration in a transwell (Millicell, Merck). The transwell was then drained and transferred to a new low glucose solution well, incubated for 1 hour, and the solution was collected as low-glucose insulin secretion. The transwell was then transferred to a high glucose solution $(11 \mathrm{mM})$ well, incubated for 1 hour, and the solution was collected as high-glucose insulin secretion. The insulin content was analyzed by murine insulin ELISA kit (Mercodia). The stimulation index (SI) was calculated as a ratio of insulin secreted in high to low glucose stimulation.

\section{Pancreatic islet transplantation and glucose tolerance test}

The impact of SA-CD47 on the modulation of IBMIR and islet engraftment was assessed in a minimal mass intraportal model. Four days before transplantation, male C57BL/6 mice (10-14 wks-old) were rendered diabetic by single intravenous injection of streptozotocin (200 mg/kg, Sigma-Aldrich). Blood glucose level were obtained using a 
portable glucose meter (Roche, AccuCheck). Animals with two consecutive non-fasting blood glucose readings of $>250 \mathrm{mg} / \mathrm{dl}$ were considered diabetic and used for intraportal transplantation. Each diabetic recipient was transplanted with 125 SA- or SA-CD47engineerd islets via portal vein. Animals were monitored for blood glucose levels twice a week. Long-term graft recipients with normoglycemia were subjected to a standard intraperitoneal glucose tolerance test (IPGTT). Briefly, animals were injected intraperitoneally with glucose bolus $(2 \mathrm{mg} / \mathrm{kg})$ after $6 \mathrm{hrs}$ of fasting and monitored for blood glucose levels at various time points $(0,10,20,30,45,60,90,120,150$ mins). Naïve C57BL/6 mice were used as controls for IPGTT.

\section{Isolation of intrahepatic immune cells}

Intrahepatic immune cells were isolated using mechanical disruption as previously described ${ }^{198}$. Briefly, after euthanasia, cardiac perfusion was performed with $10 \mathrm{ml}$ of sterile, cold saline. Mechanical disruption was done using frosted slides (in HBSS) followed by repeated passing through a18-G needle. The suspension was centrifuged at $500 \mathrm{rpm}$ for $1 \mathrm{~min}$ and supernatant was transferred to a new tube and centrifuged at 1400 $\mathrm{rpm}$ for $10 \mathrm{~min}$. The pellet was resuspended in complete RPMI, filtered, and processed for flow cytometry analysis.

\section{Flow cytometry and antibodies}

The optimal concentration for all fluorochrome-conjugated antibodies was determined by titration. Antibodies clones specific against mouse CD45.2 (eBiosciences, 12-0454-82), 
CD11b (BD Biosciences, 550993), Ly6C (Biolegend, 128036), Ly6G (Biolegend, 127606), F4/80 (Biolegend, 123122), Gr1 (eBiosciences, 48-5931) were used. For surface staining, cells were first incubated with Fc blocking antibody (anti CD16/32, Biolegend) for $10 \mathrm{~min}$ at $4^{\circ} \mathrm{C}$ followed by incubation with surface antibodies for $30 \mathrm{~min}$ at $4^{\circ} \mathrm{C}$. The cells were analyzed by BD LSR II and Flow jo (Tree Star, CA).

\section{In vitro tube model of islet-blood interactions}

In vitro tube model to mimic IBMIR was performed as described ${ }^{173}$. Fresh autologous blood was harvested and collected in heparin-coated $1.5 \mathrm{ml}$ Eppendorf tubes. SA- or SACD47-engineered islets (100 islets) were mixed and incubated with $500 \mu 1$ of blood (without any additional heparin). Tubes were incubated in a rotator at $37{ }^{\circ} \mathrm{C}$ incubator for 3 hrs. Post incubation, serum was collected, and blood-islet clots were preserved in $10 \%$ Neutral Buffered Formalin (NBF, source) followed by paraffin embedding for histological analysis.

\section{RNA isolation and quantitative real-time PCR}

Total RNA was extracted from the liver tissues or islet-thrombus using TRIzol reagents (Invitrogen Corporation, USA) according to the manufacturer's instructions. Total RNA was quantified using a NanoDrop ND-2000c spectrophotometer (Thermo Fisher Scientific, Inc.). In addition, cDNA was reverse transcribed from total RNA ( $2 \mu \mathrm{g})$ using SuperScript IV VILO cDNA Master Mix (Thermo Fisher Scientific, USA) with the following thermal conditions: $25^{\circ} \mathrm{C} 10 \mathrm{~min} ; 50^{\circ} \mathrm{C} 10 \mathrm{~min}$; and $85^{\circ} \mathrm{C} 5 \mathrm{~min}$. qRT-PCR was performed using validated TaqMan Gene Expression Assays (Table 1) according to the manufacturer's instruction on Quant Studio 3 RT-PCR system (Thermo Fisher Scientific. USA). 
Table 4. TaqMan gene expression assay for islet graft analysis

\begin{tabular}{|l|l|}
\hline Gene symbol & Assay ID \\
\hline F3 (TF) & Mm00438855_m1 \\
\hline IL1b & Mm00434228_m1 \\
\hline MCP-1 & Mm00441242_m1 \\
\hline NF-KB (p65) & Mm00501346_m1 \\
\hline GAPDH & Mm99999915_21 \\
\hline HMGB1 & Mm00849805_gH \\
\hline
\end{tabular}

\section{Histological analysis}

Paraffin-embedded clots were cut into $4 \mu \mathrm{m}$ thickness, H\&E stained, and then graded for parameters as described ${ }^{199}$. Grade 0: intact islet morphology with uniform nuclear distribution without any fragments/fractures. Grade 1, intact islet morphology with uniform nuclear distribution with minimal fragments/fractures; Grade 2, disrupted morphology with non-uniform nuclear distribution with significant fragments/fractures along with necrotic patches; and Grade 3, significant disruption of morphology with loss in nuclei and significant necrotic patches.

For immunohistochemical analysis, islets bearing liver sections or islets clots retrieved from in vitro tube assay were paraffin-embedded, then sectioned at $4 \mu \mathrm{m}$ thickness on a microtome (Leica). The sections were dewaxed and processed for histological evaluation as reported ${ }^{87,90,199}$. Sections were stained with anti CD11b (Novus biologicals, NB600-137) 
or anti-insulin (Dako, A0564) or Hoechst (Molecular probes, H-3570). Fluorescent images were obtained using a Leica confocal microscopy.

\section{Statistical analysis}

All data sets were analyzed using Graph pad prism v.7 (GraphPad Inc. CA) and expressed as mean \pm SEM. Mean comparison between the groups was performed by using Student's t-test. Survival curves were compared using the Log-rank test (Mantel-Cox) statistical method. $\mathrm{p}<0.05$ was considered significant. 


\section{Results}

\section{Generation and characterization of chimeric SA-CD47 protein}

Transient and positional display of recombinant protein ligands to immune receptors on the cell surface provides a practical and potentially safe alternative to ectopic gene expression for immunomodulation. We have developed the concept of generating recombinant immunological ligands with a modified form of core streptavidin and their transient and positional display on the surface of biotinylated cells and tissues for immunomodulation with demonstrated efficacy in autoimmunity, transplantation, and cancer settings ${ }^{129,200-204}$. Using this scheme, we herein designed a synthetic gene encoding the extracellular domain of mouse CD47 (aa 19-161), required for binding and signal transduction through $\operatorname{SIRP} \alpha$, and a modified form of core streptavidin (SA). The chimeric gene was cloned into the Drosophila copper sulfate-inducible pMT/BiP/V5-HisA expression vector with an $\mathrm{N}$-terminal secretion signal sequence $(\mathrm{BiP})$ and a hexahistidine tag (6xHis; Figure 22A). Drosophila S2 cells stably transfected with the construct used for protein expression and purification using a metal affinity chromatography as we reported previously ${ }^{129}$. SA-CD47 was run as a $\sim 37 \mathrm{kDa}$ protein in denaturing SDS-PAGE when the samples were heated at $100^{\circ} \mathrm{C}$ and as $>250 \mathrm{kDa}$ oligomers without heat treatment (Figure 22B), consistent with the reported structural feature of native streptavidin ${ }^{145}$. Western blot analysis using anti-SA antibody confirmed the identity of the protein (Figure 22C). 


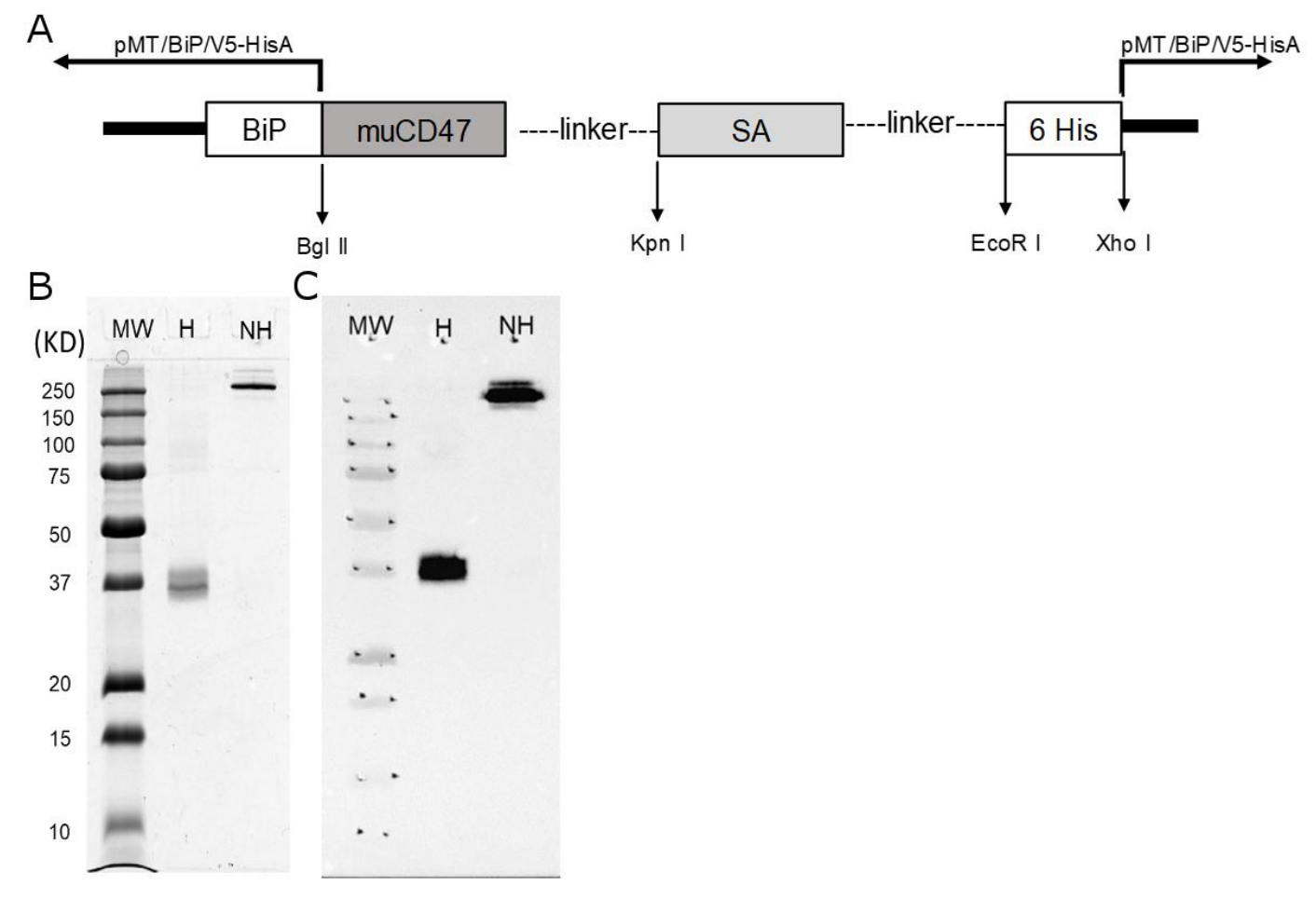

Figure 22. Cloning, expression, and structural characterization of SA-CD47 protein. (A) Schematic representation of SA-CD47 construct. A synthetic chimeric gene encoding the extracellular domain of mouse CD47 N-terminus to core streptavidin (SA) with flexible linkers and a $6 \mathrm{xHis}$ tag to facilitate protein purification was subcloned into the $\mathrm{CuSO}_{4}$ inducible pMT-Bip-V5-HisA S2 insect cell expression vector. (B) SDS-PAGE profile of the purified SA-CD47 protein. The protein was purified from culture supernatant of S2 stable transfectants using immobilized metal affinity chromatography and analyzed using SDS-PAGE after heating the samples at $100{ }^{\circ} \mathrm{C}(\mathrm{H})$ or without heat $(\mathrm{NH})$. (C) Western blot profile of SA-CD47 using antibodies against streptavidin. The SA-CD47 chimeric protein runs as a single monomeric band $(\sim 37 \mathrm{kDa})$ with heat and as oligomers (> $250 \mathrm{kDa})$ without heat. 


\section{Effective engineering of islets with SA-CD47 protein without negatively impacting their viability or function}

We next employed engineering of mouse spleen cells as a flexible and quantitative platform to assess the function of streptavidin domain of chimeric SA-CD47 protein for binding biotinylated surfaces ${ }^{145}$. Mouse spleen cells were first modified to attach a reactive biotin to the cell surface through NHS ester crosslinking followed by engineering with various concentrations of the SA-CD47 protein. There was a dose-dependent attachment of SA-CD47 to the surface of biotinylated cells that reached a plateau at $320 \mathrm{ng}$ protein $/ 10^{6}$ cells ( $\sim 99 \%$ of live cells with a mean MFI of 6260$)$ as assessed by flow cytometry (Figure 23 and 24A).

We next tested if pancreatic islets can be engineered with SA-CD47 without a detrimental effect on islet viability and function. C57BL/6 mouse islets were surface modified with biotin $(5 \mu \mathrm{M})$ followed by engineering with SA-CD47 protein (400 ng/125 islets). Confocal microscopic analysis of islets using an anti-CD47 Ab demonstrated dense display of the protein on the surface (Figure 24B). Islets were analyzed for viability and metabolic activity using Alamar Blue (AB) assay to ensure the lack of a detrimental effect due to SA-CD47 engineering. Viable and metabolically active cells reduce resazurin, a component of $\mathrm{AB}$, to resorufin by mitochondrial enzymes ${ }^{197}$. The degree of redox reaction is an indicator of the metabolic activity of viable cells ${ }^{197}$. After incubation for $6 \mathrm{hrs}$ in $\mathrm{AB}$, there was no significant difference in the level of reduced resorufin between SA-CD47engineered islets and unmodified control islets (Figure 24C), demonstrating lack of a detrimental effect causing by engineering. This observation was further corroborated with FDA/PI viability assay performed $24 \mathrm{hr}$ post-engineering. Confocal microscopy analysis 
revealed that the majority of SA-CD47-engineered islets (> 90\%) were intact and viable as indicated by bright FDA staining (Figure 24D). Engineered islets were also morphologically intact without any hypoxic centers (Figure 25). GSIS assay also showed comparable levels of functional fitness between SA-CD47-engineered islets and unmodified control islets in insulin secretion (Figure 24E). Importantly, no difference in stimulation indices of SA-CD47-islets and unmodified islets were observed (Figure 24E). Taken together, these results demonstrate that pancreatic islets can be efficiently engineered to display the SA-CD47 protein on their surface without a negative impact on their viability, metabolic activity, and insulin secretion function. 


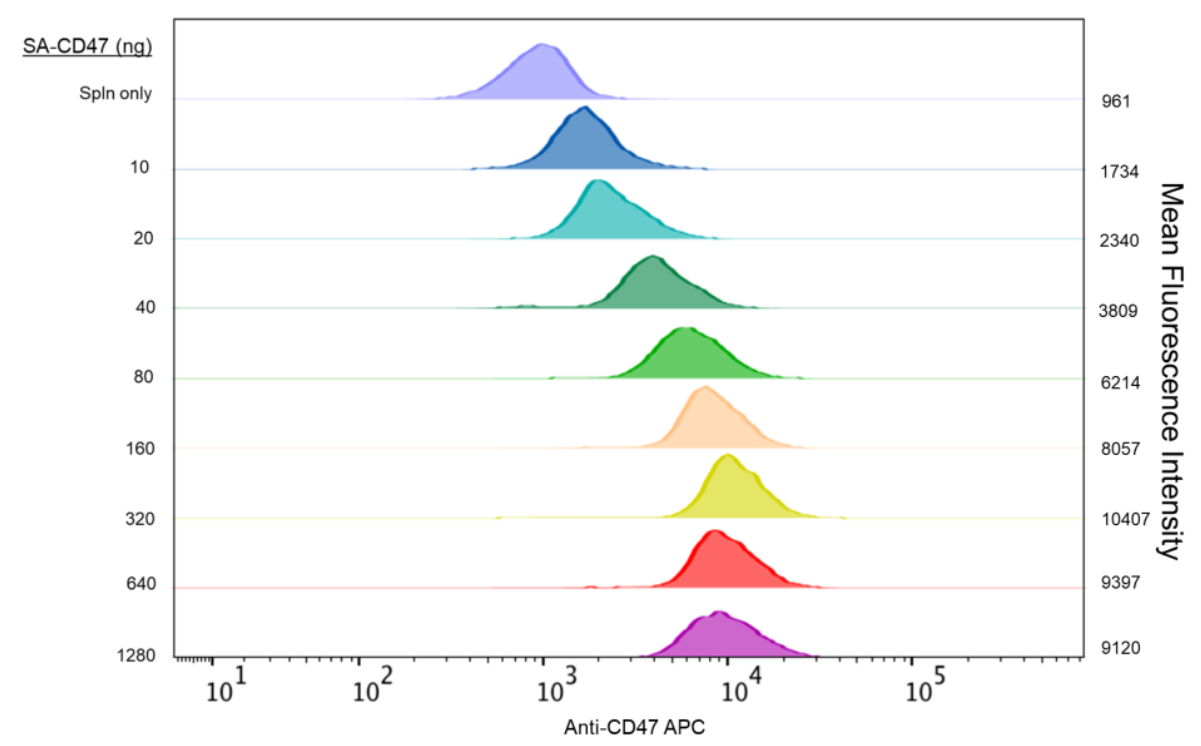

Figure 23. Representative histogram overlay plot of spleen cells engineered with SACD47 protein. Spleen cells were biotinylated $(5 \mu \mathrm{M})$ and then engineered with SACD47 protein at the indicated concentrations (ng protein $/ 10^{6}$ cells). Histograms in color show the fluorescence intensity (MFI) at respective engineering levels. 


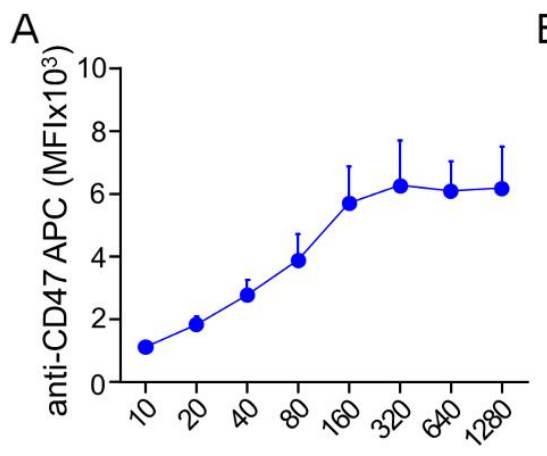

$\mathrm{SA}-\mathrm{CD} 47(\mathrm{ng})$

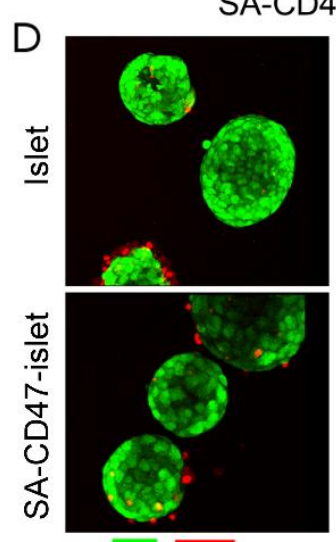

Live Dead
B

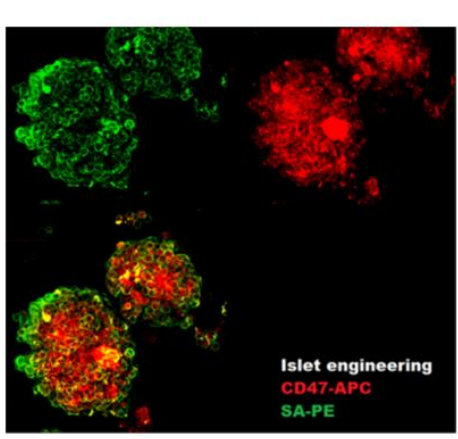

$\mathrm{E}$

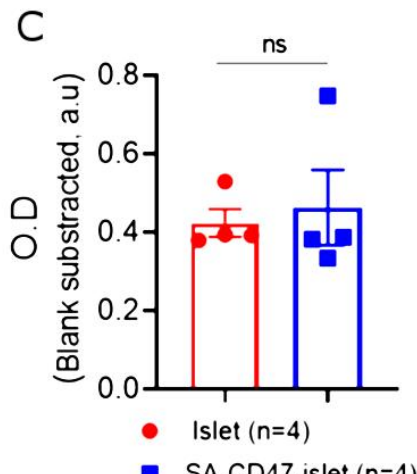

- SA-CD47-islet $(n=4)$
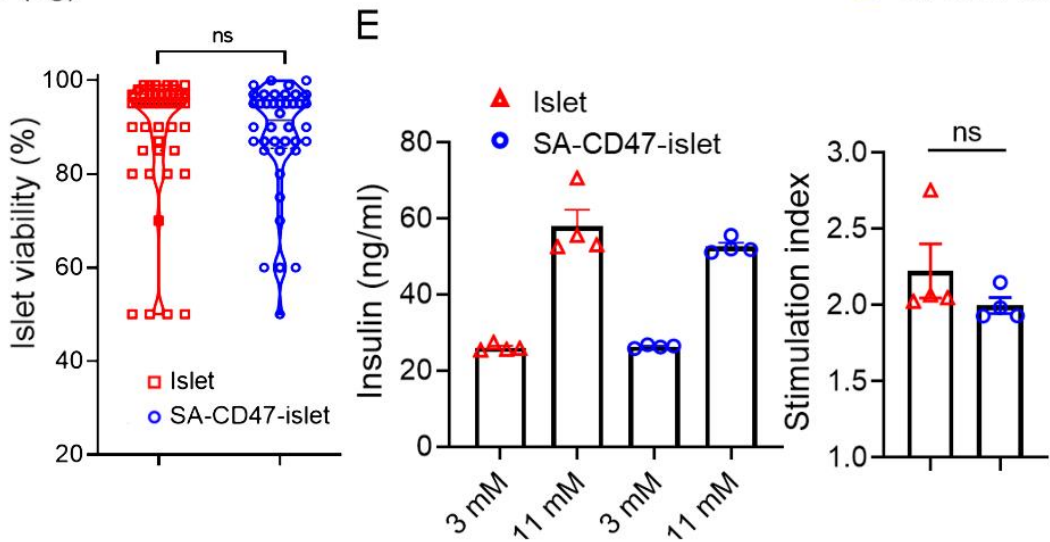

Figure 24. Effective engineering of islets with SA-CD47 protein without a negative impact on their viability or function. (A) Assessment of cell surface engineering with SA-CD47 protein. Spleen cells were biotinylated in $5 \mu \mathrm{M}$ EZ-Link Sulfo-NHS-LC-Biotin followed by engineering with the indicated amounts of the SA-CD47 protein (ng/10 6 cells). The levels of chimeric protein on the cell surface was assessed using an anti-CD47 antibody in flow cytometry. The MFI values as a function of protein concentrations from three independent experiments are graphed (B) Representative image of islet engineered with SA-CD47 protein. Mouse islets were biotinylated $(5 \mu \mathrm{M})$ followed by engineering with SA-CD47 protein (400 ng/125 islets). The levels of biotin and CD47 molecules were assessed using SA conjugated PE (SA-PE; green) and an antibody against CD47 (antiCD47 APC; red), respectively, in confocal microscopy. (C) Engineering with SA-CD47 
protein does not impact islet viability and metabolic activity. SA-CD47-engineered islets (SA-CD47-islet) or unmodified naïve islets (Islet) were incubated with alamar blue reagent for 6 hours at $37^{\circ} \mathrm{C}$. The level of alamar blue reduction was measured as absorbance at 562 nm. Data are from two independent experiments. (D) FDA/PI viability test showing lack of a detrimental effect of engineering on islets. Representative live/dead confocal microscopy images of unmodified and SA-CD47-engineered islets 24 hours post engineering $($ green $=$ live; red $=$ dead $)$. A total of 47 unmodified $($ Islet $)$ and 40 engineered (SA-CD47-islet) islets were analyzed for viability. (E) Glucose-induced insulin secretion assay showing functional fitness of SA-CD47-engineered islets. Data expressed as mean \pm SEM. For comparison of means, an unpaired t-test was used for C, D and E. ns, not significant. 

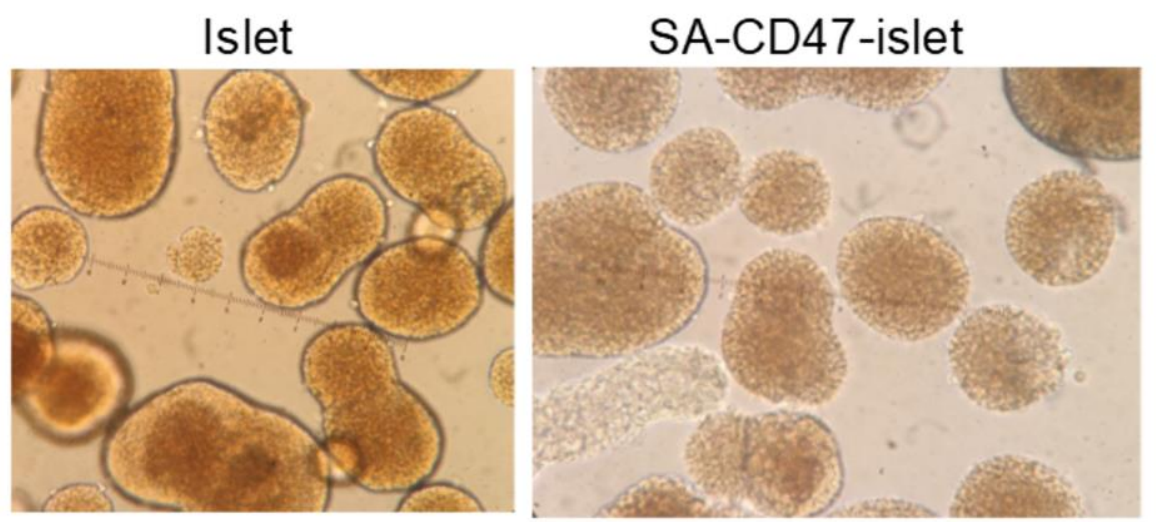

Figure 25. Bright field images showing integrity of SA-CD47-engineered islets.

Unmodified (control, left) and SA-CD47-engineered islets (right) were cultured for 24 hours post engineering and then imaged. 


\section{SA-CD47-engineered xenogeneic cells are refractory to phagocytosis by}

\section{macrophages.}

CD47 expressed on autologous cells deliver a "don't eat me" signal through $\operatorname{SIRP} \alpha$, a negative regulator of phagocytosis, expressed on macrophages and dendritic cells ${ }^{119}$. Interaction of CD47 with $\mathrm{SIRP} \alpha$ is species-specific and dictates the phagosome formation $^{205}$. Thus, we assessed the anti-phagocytic function of SA-CD47 in a standard xenogeneic in vitro phagocytosis assay ${ }^{12,183}$. Rat splenocytes were engineered with SACD47 or SA as a control protein (Figure 26A) and then labeled with the fluorescence dyes CTV or CFSE, respectively. Coculturing these cells with the mouse RAW 264.7 macrophage cell line demonstrated significant $(\mathrm{p}=0.0088)$ phagocytosis of SA-engineered rat cells over SA-CD47-engineered cells (Figures 26B and 27). To further corroborate this observation, we performed competitive phagocytosis assay in mixed culture settings where RAW 264.7 cells were co-cultured in the presence of both target cells labelled with different fluorescence dyes. Mouse macrophage preferentially phagocytosed SA-rat spleen cells (Figures 26C and 27). Collectively, these results demonstrate that the SA-CD47 protein is functional and when displayed on target cells prevents phagocytosis by macrophages. 


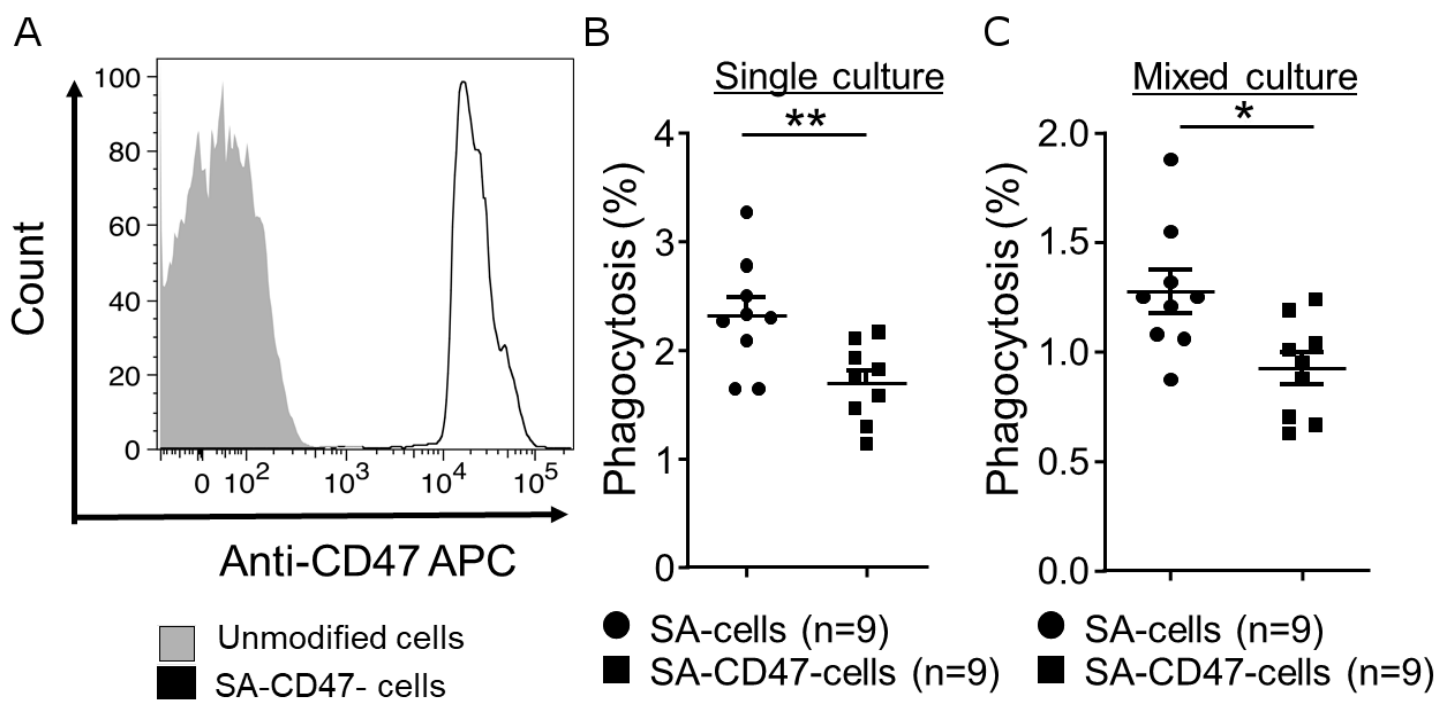

Figure 26. SA-CD47 protein on xenogeneic cells prevents phagocytosis by macrophages. (A) A representative histogram of rat splenocytes engineered with the SACD47 protein. Rat splenocytes engineered with the SA-CD47 protein (200 ng/10 6 cells) were analyzed using an antibody against mouse CD47 in flow cytometry (dark line open histogram) with unmodified cells (gray shaded histogram) serving as control. (B) SACD47 inhibits phagocytosis of rat splenocytes by macrophages. Mouse RAW 264.7 macrophage cells were cultured with CFSE-labelled SA-engineered rat splenocytes or CTV-labelled SA-CD47-enginered rat splenocytes at 1:5 ratio. After 18 hours of incubation, cells were analyzed by flow cytometry by gating on $\mathrm{CD}_{1} 1 \mathrm{~b}^{+} \mathrm{CFSE}^{+}$or

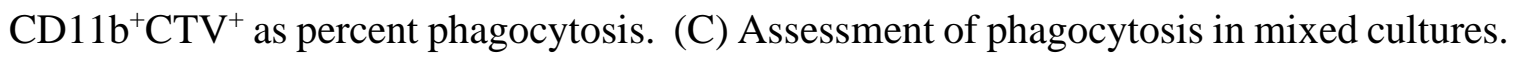
Rat cells engineered with SA and SA-CD47, and labelled with CFSE and CTV, respectively, were mixed at 1:1 ratio and then co-cultured with RAW 264.7 cells under the same conditions for (B). Phagocytosis of rat cells by macrophages was assessed using flow 
cytometry. Data shown as mean \pm SEM of three independent experiments. ${ }^{*} p<0.05,{ }^{* *} \mathrm{p}$ $<0.01$; two-tailed unpaired t-test.
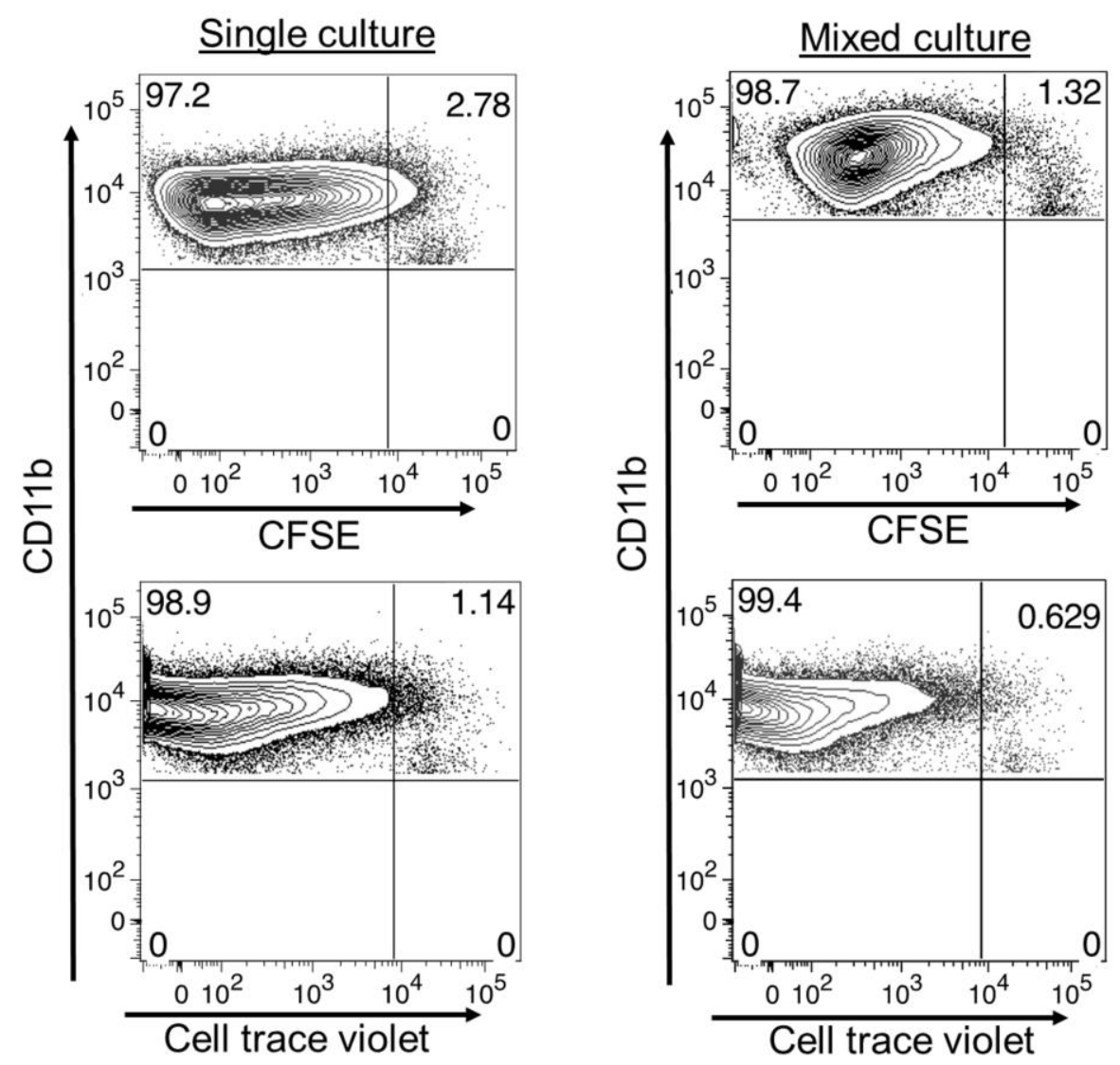

Figure 27. Representative flow plots of in vitro phagocytosis assay. SA-CD47engineerd rat splenocytes (CTV labelled) are more refractory to phagocytosis by mouse macrophages as compared with SA-engineered cells in single or competitive mixed culture settings. 


\section{SA-CD47 protects islets and prevents intra-islet infiltration of CD11 ${ }^{+}$cells in an in}

vitro loop assay.

First, to understand the potential role of CD47 in protection of islets from IBMIR mediated destruction, we performed a modified in vitro loop assay for islet-blood interaction ${ }^{173}$. Engineering with SA-CD47 did not prevent fibrin deposition or thrombosis after incubation with blood. However, histological analysis of the islet-thrombus revealed relatively intact SA-CD47-engineered islets, whereas SA-engineered islets had significant $(\mathrm{p}=0.0128)$ destruction (Figure 28A). Intact islets with regular morphology with uniform distribution of nuclei (Grade 0) were more common in SA-CD47-engineered islets (40\%) as compared with the SA-engineered control group (25\%) (Figure 28A). SA-engineered group had more Grade 2 islets with disrupted morphology with necrotic centers and Grade 3 islets with highly disrupted morphology as compared with SA-CD47-engineered group (Grade 2: 25\% vs. 18\%; Grade 3: $24 \%$ vs. 11\%). Immunofluorescence analysis of isletthrombus sections demonstrated significantly $(\mathrm{p}=0.0244)$ higher infiltration of CD11 $\mathrm{b}^{+}$ cells in SA-engineered islets as compared with SA-CD47 group (Figure 28B and 29). Collectively, these results show that SA-CD47 protects islets from IBMIR mediated disruption, potentially by inhibiting the intraislet infiltration of inflammatory cells. 


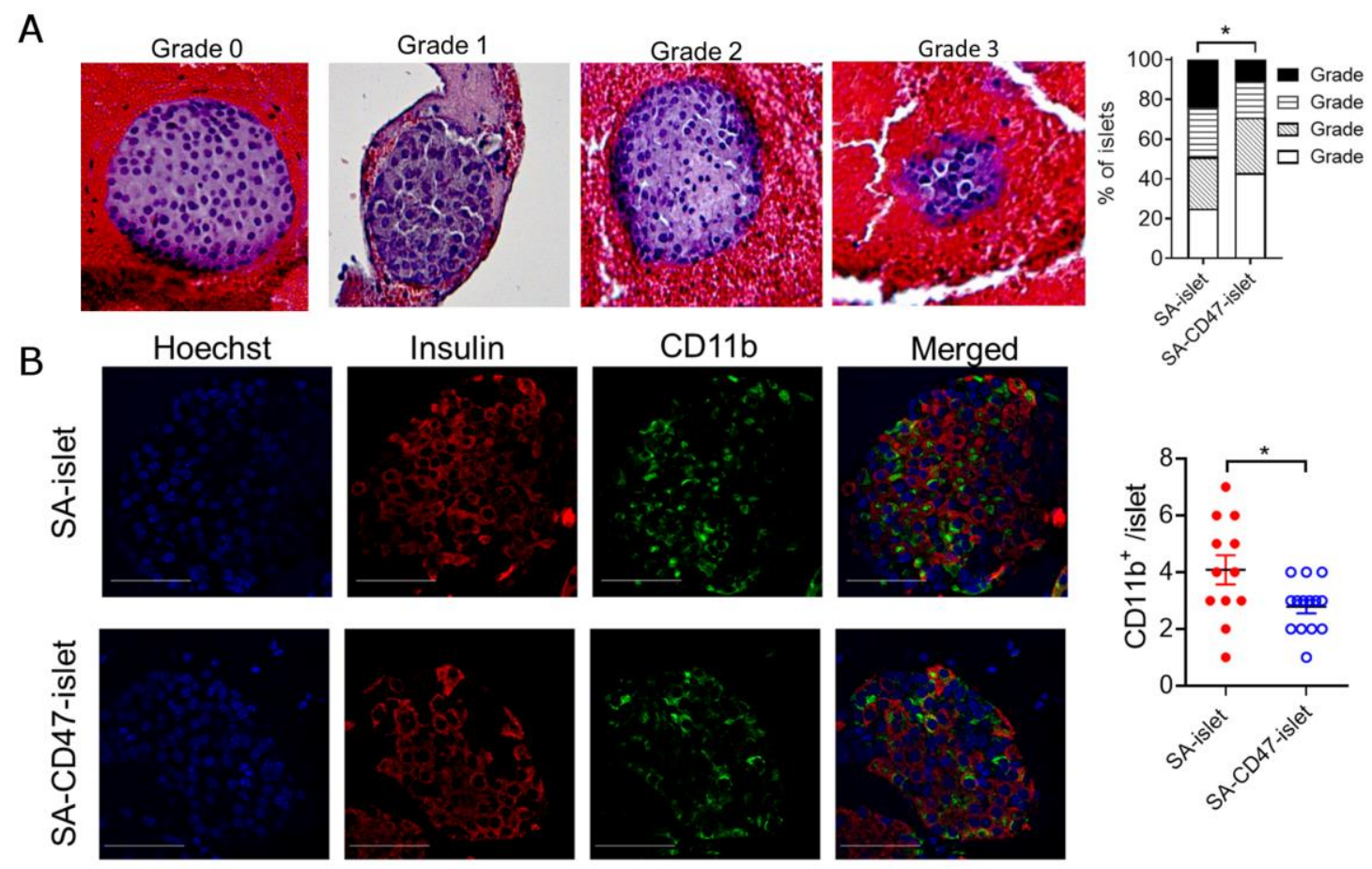

Figure 28. SA-CD47 engineering protects islets from destruction by IBMIR in an in vitro loop assay. (A) Histological grading of islets 3 hours post in vitro loop assay indicates SA-CD47-engineered islets are protected from blood mediated destruction as compared with SA-engineered control islets. Frequency distribution between the groups was analyzed by chi-square test. (B) Immunofluorescence analysis for intra-islet CD11 $\mathrm{b}^{+}$ inflammatory cells. SA-CD47-engineered islets had significantly less infiltration of $\mathrm{CD}_{11 b^{+}}$granulocytes/macrophages as compared to SA-engineered islets. Data pooled from three independent experiments and represented as mean \pm SEM. Data means compared by unpaired two-tailed t-test. $* \mathrm{p}<0.05$. 

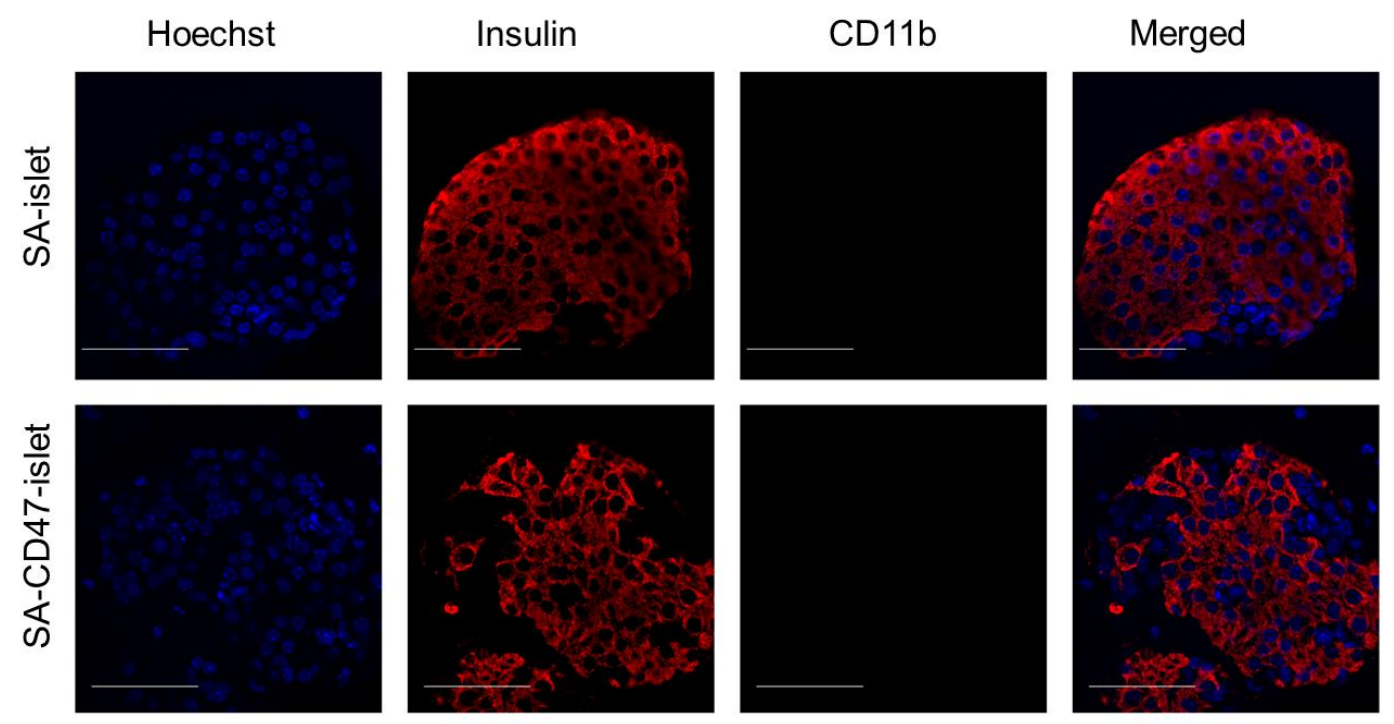

Figure 29. Representative confocal images for CD11b negative staining. Tissue sections were stained with Hoechst, insulin but without CD11b for specificity of anti CD11b antibody (scale bar $=50 \mu \mathrm{m})$. 


\section{SA-CD47-engineered islets show enhanced engraftment and function following intraportal transplantation}

Instant blood-mediated inflammatory reaction (IBMIR) occurs following the exposure of islet grafts to recipient blood and is responsible for $50-70 \%$ of islet loss immediate post intraportal transplantation ${ }^{79,173}$. Phagocytes play an important role in IBMIR $^{80,84,87,173}$. To assess the impact of SA-CD47 as an inhibitor of phagocytosis on islet engraftment and long-term function, C57BL/6 islets were engineered with SA-CD47 or SA as a control protein and used for intraportal transplantation in a syngeneic marginal mass (125 islets) model (Figure 30A). Seven out of 8 mice (87.5\%) transplanted with SACD47-engineered islet graft showed engraftment and long-term function as assessed by blood glucose levels as compared with $1 / 7$ (14.28\%) of the SA-engineered islet graft recipients $(\mathrm{p}=0.0088$; Figure $30 \mathrm{~B}, \mathrm{C}$ and 31$)$. The average days required for achieving normoglycemia was $17.86 \pm 4.9$ (mean \pm SEM) in SA-CD47-islet recipients.

Intraperitoneal glucose tolerance (IPGTT) test showed significantly rapid glucose clearance in long-term (>80 days) recipients of SA-CD47-engineered as compared with SA-engineered islet grafts (Figure 32A). Indeed, long-term SA-CD47-engineered islet graft recipients had a glucose clearance response comparable to naïve animals with a similar area under the curve (Figure 32B). Histological analyses demonstrated insulinsecreting functional islet mass in long-term recipients (Figure $32 \mathrm{C}$ and D). Collectively, these results demonstrate that the transient display of SA-CD47 protein on islet surface significantly enhance engraftment and long-term function following intraportal transplantation. 


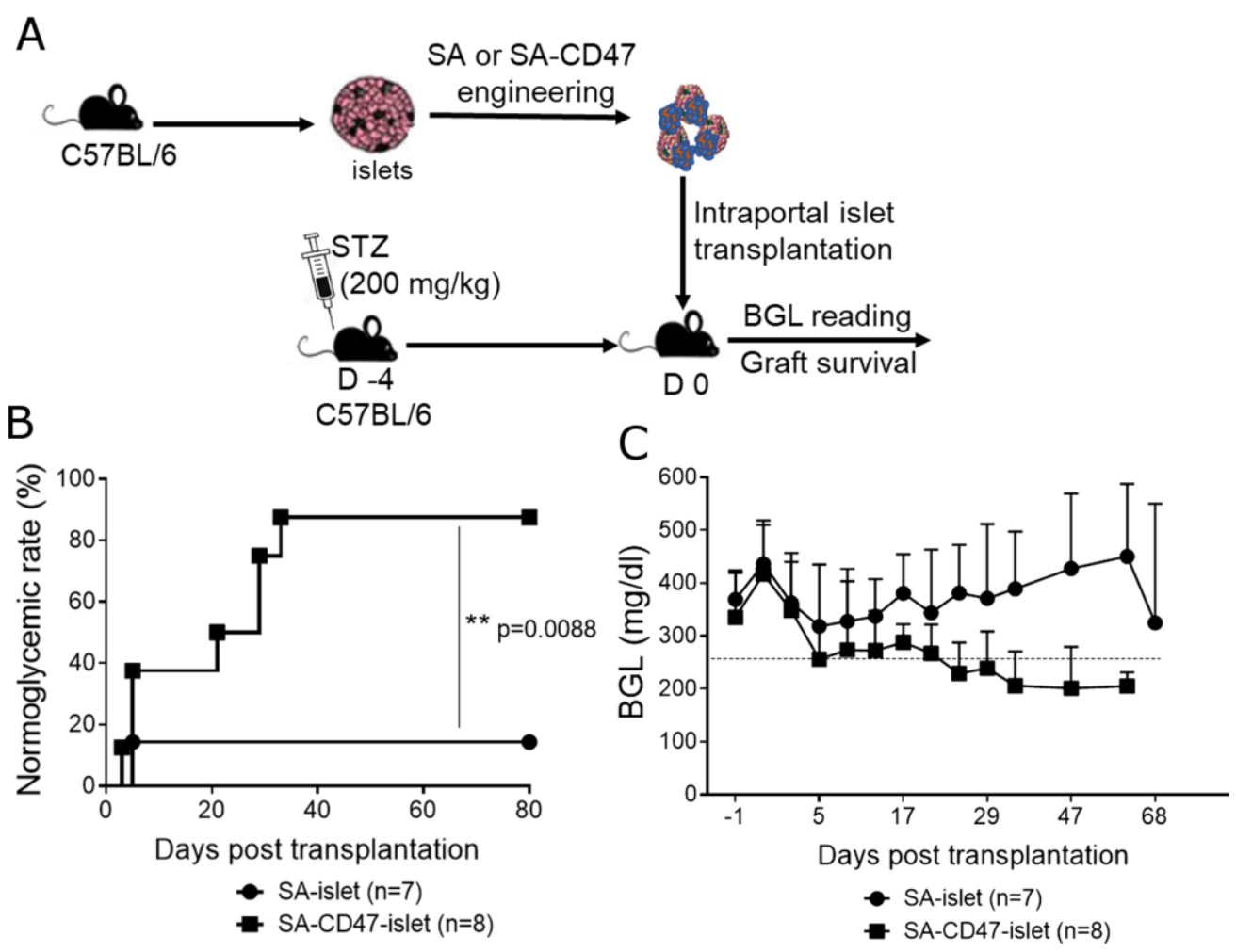

Figure 30. Surface display of SA-CD47 protein significantly improves pancreatic islet engraftment and function in a syngeneic marginal mass intraportal transplantation model. (A) Study design for intraportal islet transplantation. Animals were monitored for blood glucose levels (BGL) post-transplantation and two consecutive daily readings of glucose $\geq 250 \mathrm{mg} \mathrm{dl}^{-1}$ was considered as engraftment failure. (B) Kaplan-Meier analysis between the groups shows significantly higher rate of post-transplant normoglycemia in chemically diabetic recipients of SA-CD47-enginered as compared with SA-engineered control grafts. Statistical difference assessed using log-rank (Mantel-Cox) test with $* *$ p $<0.01$. (C) Non-fasting blood glucose level of transplant recipients in (B). 
A

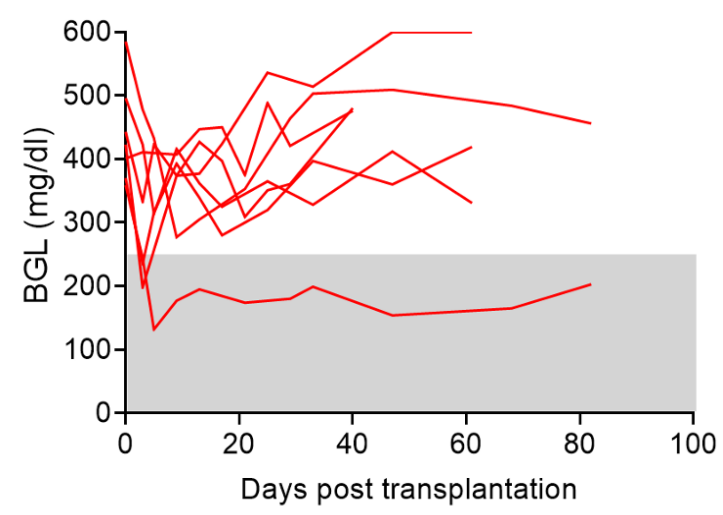

B

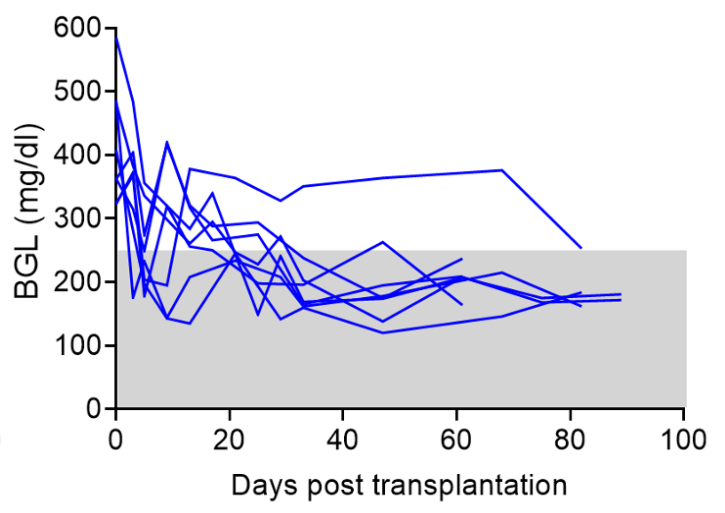

Figure. 31. SA-CD47 engineering improves the intraportal islet transplantation outcome. STZ induced $(200 \mathrm{mg} / \mathrm{kg})$ diabetic animals were transplanted with islets engineered with SA (A, $n=7)$ or SA-CD47 protein $(B, n=8)$. Animals were monitored for blood glucose levels $(\mathrm{mg} / \mathrm{dl})$ post transplantation. Grey shaded area indicates the euglycemic region (BGL $<250 \mathrm{mg} / \mathrm{dl})$. 

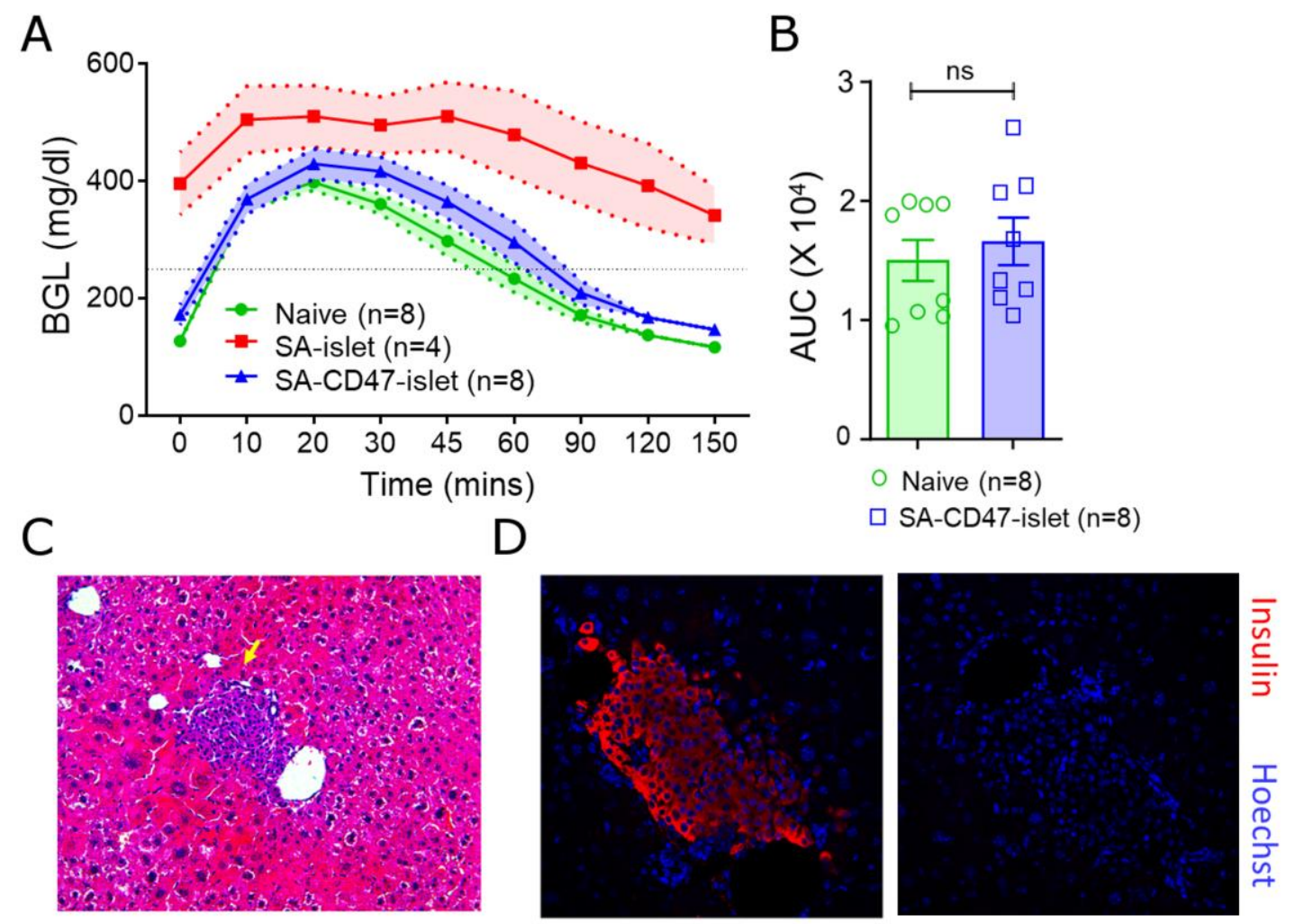

Figure 32. SA-CD47-engineered islet grafts show long-term survival and function following intraportal transplantation. (A) Intraperitoneal glucose tolerance test shows functional islet mass in long-term (>80 days) recipients of SA-CD47-engineered islet grafts. SA-CD47-engineered islet graft recipients (blue) were able to regulate blood glucose at levels comparable to age-matched naïve animals (green). In marked contrast, SA-engineered islet graft recipients (red) failed to regulate blood glucose levels following intraperitoneal glucose challenge. (B) Area under the curve analysis for (A). (C, D) Histological analyses showing insulin positive islets in the liver of long-term graft recipients. Representative H\&E (C) and immunofluorescence staining (D) of liver tissues of long-term recipients of SA-CD47-engineered islet grafts. Nuclei are stained with Hoechst (blue) and $\beta$-cells with anti-insulin antibody (red). Data mean comparison was done by two-tailed unpaired t-test for B. Shadow indicate SEM; ns, not significant. 


\section{SA-CD47 display alters the intrahepatic infiltration of inflammatory cells and inflammatory mediators.}

One of the characteristic features of IBMIR mediated early loss of islets is the recruitment of inflammatory myeloid cells into the site of engraftment ${ }^{87,97}$. To test whether SA-CD47 on the surface of islets protect them from IBMIR mediated destruction, livers of graft recipients were analyzed using flow cytometry to assess intrahepatic inflammatory infiltrates 3 hrs post-transplantation. Livers of recipients transplanted with SA-CD47engineered islet grafts had lower numbers of inflammatory infiltrates, including CD11 $\mathrm{b}^{+}$ myeloid cells, inflammatory monocytes $\left(\mathrm{CD} 11 \mathrm{~b}^{+}\right.$Ly6C $^{\text {hi }} / \mathrm{CD}_{11 b^{+}}$Ly6C $\left.^{\text {int }}\right)$, neutrophils $\left(\mathrm{CD} 11 \mathrm{~b}^{\mathrm{hi}} \mathrm{Gr} 1^{\mathrm{hi}}\right)$, and macrophages $\left(\mathrm{F} 4 / 80^{+}\right)$as compared with SA-engineered islet controls (Figure 33A and 34). However, when analyzed at $24 \mathrm{hrs}$ post transplantation, there were similar levels of intra hepatic inflammatory infiltrates in both SA-CD47- and SAengineered islet graft recipients. This observation is in line with previous studies reporting appearance of inflammatory infiltrates in islet grafts as early as 15 min with massive infiltration by 2 hrs post transplantation ${ }^{84,87,89}$.

We further analyzed the liver tissue samples by quantitative RT-PCR to assess the expression of inflammatory mediators involved in peri-transplant islet mass loss. There was no significant difference in transcript levels for various inflammatory mediators between SA-CD47- and SA-engineered islet graft recipients when assessed at $3 \mathrm{hr}$ post transplantation. In marked contrast, when analyzed 24 hrs post transplantation, liver samples from SA-engineered islet recipients had significantly higher expression of transcripts for high mobility group box-1 (HMGB-1), tissue factor (TF), IL-1 $\beta$, which are responsible for early islet loss as compared with the SA-CD47-engineered islet group 
(Figure 33B). Although there was reduction in transcripts levels for $\mathrm{MCP}-1$ and $\mathrm{NK}-\kappa \mathrm{B}$ in the SA-CD47-engineerd islet group as compared with the SA-engineered group, differences did not reach statistical significance. Taken together, these results show that the presence of SA-CD47 protein on islet grafts mitigates IBMIR by reducing the infiltration of inflammatory cells and their expression of inflammatory factors, resulting in enhanced engraftment and sustained function. 


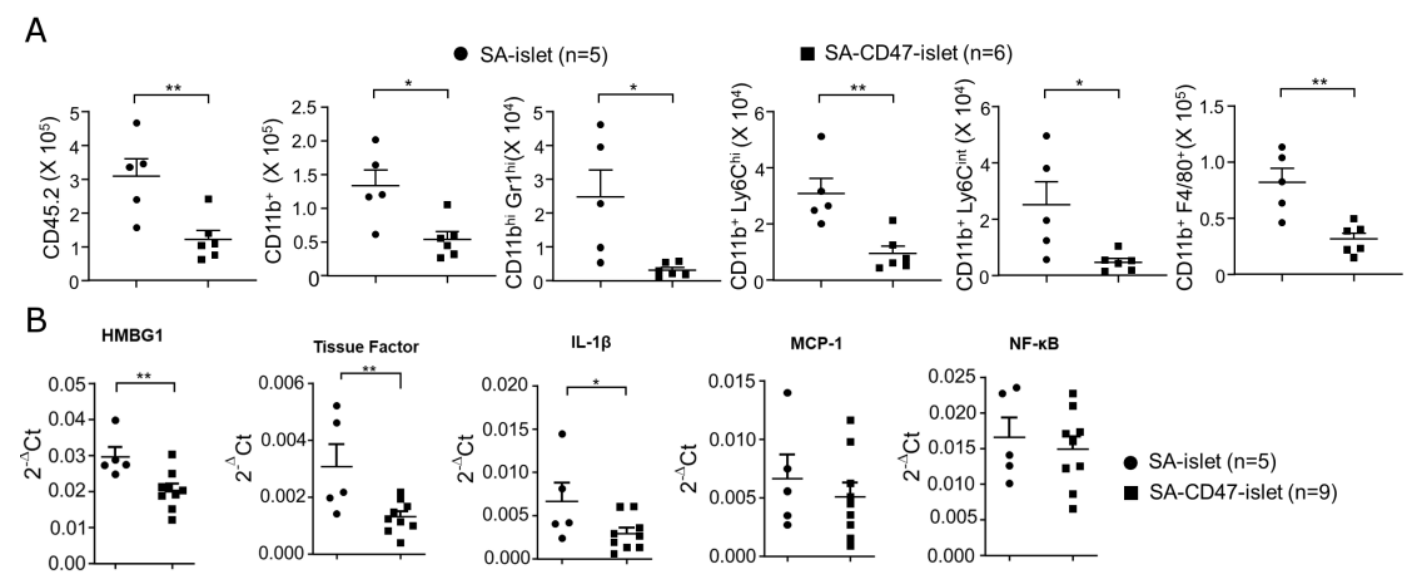

Figure 33. SA-CD47-islet recipients have significantly reduced infiltration of inflammatory cells and mediators in liver following intraportal transplantation. (A) Intrahepatic immune cells analysis $3 \mathrm{hr}$ post-transplantation show substantially less infiltration of immune cells in SA-CD47-engineered islet graft recipients as compared with control SA-engineered islet recipients. Data points represent absolute cell number per gram of liver pooled from two independent experiment with $n=2-3$ per group. (B) RTqPCR analysis of liver tissue samples $24 \mathrm{hr}$ post-transplantation shows heightened expression of inflammatory mediators in SA-engineered islets as compared with SA-CD47-engineered islets. Data points represent relative expression in comparison to GAPDH pooled from two independent experiments, each with $n=2-4$ per group. For comparison of means unpaired one-tailed t-test was used in (A) and (B). *p $<0.05,{ }^{*} \mathrm{p}<0.01$. Data shown as mean \pm SEM. 

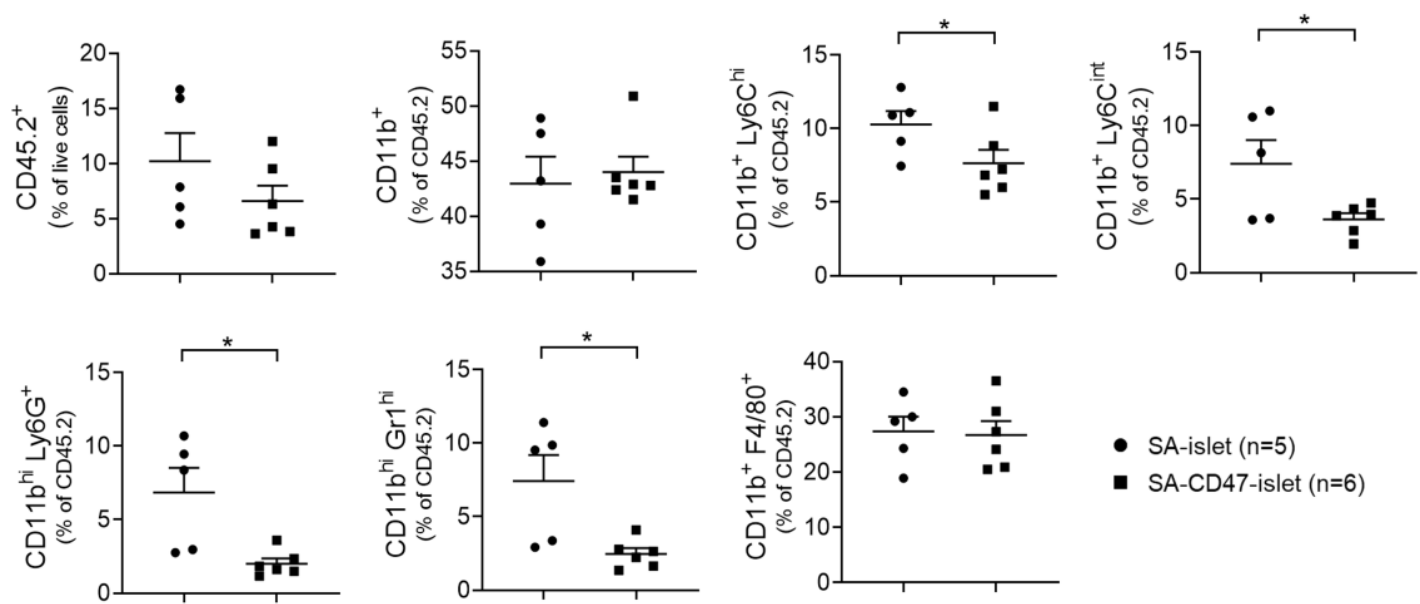

Figure 34. SA-CD47-engineered islet graft recipients have substantially less intrahepatic infiltration of inflammatory cells. Data point indicates frequency of respective cells (as frequency of total CD45.2 $2^{+}$cells) 3 hrs post-transplantation. Data pooled from two independent experiments, with $n=2-3$ in each group, represented as mean \pm SEM. $* p<0.05$, $* * p<0.01$. For comparison of means unpaired one-tailed $\mathrm{t}$-test was performed. 


\section{Discussion}

IBMIR mediated primarily by myeloid cells is responsible for significant islet mass loss immediate post infusion into the liver ${ }^{174-176}$. Despite being transplanted from multiple donors, the functional islet mass in insulin-independent patients is equivalent of $30 \%$ of a non-diabetic healthy individual ${ }^{90}$. CD47/SIRP $\alpha$ axis is a critical innate immune checkpoint that inhibits activation and phagocytic function of myeloid cells as a critical mechanism of self/non-self-discrimination ${ }^{186}$. Tumor cells express CD47 on their surface as an effective means of immune evasion and the blockade of this molecule using antibodies was shown to galvanize innate immune responses against tumor with remarkable efficacy for the clearance of cancer in various preclinical models ${ }^{186-189}$. In this study, we assessed whether tumor immune evasion mechanisms can be simulated using CD47 as a means of mitigating IBMIR. We herein report for the first time that the transient display of a novel form of this molecule, SA-CD47, on the surface of islets results in enhanced engraftment and long-term function in an intraportal minimal mass syngeneic mouse model. Long-term engraftment and function were associated with peri-transplant reduced intragraft inflammatory innate immune cells and transcripts for proinflammatory mediators.

As a practical and alternative approach to ectopic expression of CD47 in islets, we generated a novel construct, SA-CD47, that contained the extracellular functional domain of mouse CD47 chimeric with core-streptavidin using a previously published scheme for FasL as a regulator of adaptive immunity ${ }^{129}$. Consistent with the structural features of streptavidin $^{206}$, SA-CD47 protein exists as oligomers and was displayed on the surface of biotinylated cells and islets in a rapid and efficient manner taking advantage of the high affinity interaction $\left(\mathrm{K}_{\mathrm{d}} \sim 10^{-14} \mathrm{M}\right)$ between biotin and streptavidin ${ }^{206}$. Importantly, the 
engineering of islets with SA-CD47 did not negatively impact their viability, metabolic activity, or insulin secretion. These findings are consistent with our published studies transiently displaying SA-FasL on the surface of islets for the regulation of alloreactive immune responses ${ }^{204,207}$. In an in vitro xenogeneic system, SA-CD47-engineered rat cells overcame phagocytosis by mouse macrophages. This observation is consistent with studies demonstrating that porcine cells genetically modified to express human CD47 circumvent phagocytosis by human macrophages ${ }^{195}$. Engraftment of human hematopoietic stem cells is sensitive to SIRP $\alpha$ alleles expressed in mice as NOD expressing the high affinity allele for human CD47 show better engraftment as compared with mice expressing alleles with low affinity ${ }^{194}$. Furthermore, mouse hematopoietic stem cells were shown to transiently upregulate $\mathrm{CD} 47$ on their surface in response to mobilizing cytokines and inflammatory agents and the level of expression was directly correlated to their ability to evade phagocytosis $^{208}$.

In vitro loop assay that simulates in vivo IBMIR, that involves direct contact of peripheral blood with islets, showed well-preserved morphology with uniform distribution of nuclei and minimal fracture of SA-CD47-engineered islets as compared with SAengineered controls. There were significantly $(\mathrm{p}=0.0244)$ fewer $\mathrm{CD} 11 \mathrm{~b}^{+}$cells in the isletthrombus as compared with SA-engineered islets. This result suggested that SA-CD47 can potentially abrogate IBMIR effects. Following this observation, in intraportal islet transplantation, islets engineered with SA-CD47 protein showed significantly enhanced engraftment and long-term function (87.5\%) as compared with SA control proteinengineered islets (14.2\%) following intraportal transplantation. These observation provides strong evidence for the ability of SA-CD47 to mitigate IBMIR and is consistent 
with a study reporting improved engraftment of human hepatocytes transduced with the mouse CD47 in immunodeficient mice following intraportal transplantation ${ }^{209}$. Also, transplantation of hepatocytes from CD47 knockout mice into syngeneic wild type recipients resulted in the activation of macrophages and poor graft survival ${ }^{120}$. Previous studies have shown that IBMIR is initiated immediately after islet infusion ${ }^{80,84,87}$. Rapid platelets deposition is observed within 30 mins on the islet surface along with infiltration of neutrophils/macrophages $\left(\mathrm{CD} 11 \mathrm{~b}^{+}\right)$that appear as early as 15 min post-transplantation and peak at $2 \mathrm{hr}^{87}$. When analyzed at $3 \mathrm{hrs}$ post-transplantation, SA-CD47-engineered islet graft recipients had significantly less intragraft inflammatory cells, particularly inflammatory monocytes $\left(\mathrm{CD} 11 \mathrm{~b}^{+}\right.$Ly6C $\left.\mathrm{Ch} / \mathrm{Ly} 6 \mathrm{C}^{\mathrm{int}}\right)$, neutrophils $\left(\mathrm{CD} 11 \mathrm{~b}^{+} \mathrm{Gr} 1^{\mathrm{hi}}\right)$, and macrophages $\left(\mathrm{CD} 11 \mathrm{~b}^{+} \mathrm{F} 4 / 80^{+}\right)$as compared with SA-engineered controls. SA-CD47engineered islet grafts also had significantly reduced intragraft expression of proinflammatory HMGB1, TF, and IL-1 $\beta$, all have been implicated in early islet loss $79,83,210,211$. These observations indicate that the presence of SA-CD47 protein on the surface of islets protects them from IBMIR by preventing transmigration of inflammatory cells and reducing inflammatory mediators in the graft site.

The current study offers a novel, effective, and practical strategy to ectopic expression of CD47 for the enhancement of autologous islet engraftment and long-term function following infusion into the liver. However, the application of this facile approach is not limited to islets or autologous transplantation as it can be applied to any cellular auto or allotransplantation. Although in the present study, we focused on the efficacy of SACD47 in modulating innate immune responses, CD47 has also been implicated in regulating adaptive immune responses. $\mathrm{CD} 47$ interaction with $\operatorname{SIRP} \alpha$ on DCs activates 
STAT3, a critical regulator of IL-6, IL-10 and IDO expression, resulting in polarization of $\mathrm{T}$ cells toward a regulatory phenotype $\mathrm{e}^{212,213}$. Interaction of CD47 with SIRP $\alpha$ expressed on DCs inhibits their activation and impair Th1 response ${ }^{214}$. CD47 interaction with SIRP $\alpha$ on DC was shown to be critical to cardiac allograft tolerance achieved by CD154 blockade and donor-specific transfusion ${ }^{215}$. Lack of $\mathrm{CD} 47$ on donor cells resulted in rapid, alloantigen-independent activation of DCs, uncontrollable with CD154 blockade. Furthermore, intrasplenic infusion of CD47-deficinet allogeneic hepatocytes resulted in accelerated rejection of donor-matched skin grafts, whereas CD47-competant hepatocytes enhanced skin allograft survival that was associated with reduced alloreactive $\mathrm{T}$ cell responses, enhanced production of regulatory cytokines, IL-4 and IL-10, as well as significant expansion of myeloid-derived suppressor cells (MDSC) ${ }^{216}$. Indeed, CD47 was shown to play a critical role in the expansion and regulatory function of MDSC in a costimulatory blockade-induced tolerance to kidney allografts. The blockade of CD47/SIRP $\alpha$ pathway in this model resulted in rejection of long-term tolerant kidney grafts that was associated with overexpression of MCP-1 and inflammatory macrophage signature $^{217}$. However, it remains to be investigated if the transient display of SA-CD47 on allogeneic islets or cellular grafts is sufficient as a single agent or in combination with a modulator of adaptive immunity, such as SA-FasL ${ }^{204,207,218,219}$, is effective in inducing tolerance. 


\section{CHAPTER 4}

\section{SUMMARY, IMPLICATION AND FUTURE DIRECTION}

In this dissertation, we show transient display of functional chimeric proteins as a novel immunomodulatory approach in two distinct experimental models. In chapter two, we use previously reported novel form of FasL, SA-FasL, to engineer donor graft in hematopoietic stem cell transplantation. In haploidentical and xenogeneic GVHD models, we provide evidence that engineering donor graft with SA-FasL can efficiently attenuate the GVHD causing ability of the graft, potentially by eliminating alloreactive cells. This strategy involves relatively very short procedure and is clinically translatable. Unlike previously reported strategies that involves ex vivo culture of donor grafts with recipient antigens for days, this method involves very minimal modulation of donor grafts. This strategy is highly impactable for procedures involving HSCT for leukemic patients or to establish donor chimerism for solid organ allo-graft transplantation. However, resistance of some T effector cells from AICD cannot be ignored. Also, SA-FasL-spleen recipients contract GVHD at some degree and some animals recovered as such the total protection is not $100 \%$. This may be beneficial with respect to graft versus leukemia or graft versus tumor effect, where GVHD at some degree is required to maintain anti-leukemia effect. In addition, this may be important for other $\mathrm{T}$ cell functions like efficient engraftment and immunity against infections. 
The proposed strategy can be modified in multiple ways to increase the impact. First, we show that engineering whole grafts leads to significant depletion of Treg cells without a negative impact on the efficacy of the protocol in our model. However, this may not be the case in the clinic. To avoid this potential pitfall, our approach could be used in combination with other clinically applied strategies, which is attractive and easily achievable given the efficient and practical nature of engineering. For example, we have shown that rapamycin as an immunoregulatory drug with beneficial impact on Treg cells, works in synergy with SA-FasL in islet and heart transplantation models ${ }^{130,220}$. Rapamycin is used widely in the clinical transplantation with great safety profile and can be used in combination with SA-FasL engineered cells to mitigate GVHD. Alternatively, SA-FasL can be used in combination with IL-33, another immunoregulator with beneficial effect on Treg cells, or chemotherapy-based conditioning, such as cyclophosphamide that also positively impacts Treg cells and extensively used for HSCT in the clinic.

On the second part of the dissertation, we report novel implication of CD47-SIRP $\alpha$ axis in attenuation of IBMIR. We show that islets engineered with SA-CD47 can modulate innate immune responses, resulting in the prevention of early islet loss, maintaining functional islet mass, and promoting efficient engraftment. This strategy has significant implication in islet transplant settings either in allogeneic or autologous transplantation. This is the first study of its kind where we target innate immune cells, myeloid cells, to modulate immune response to prevent early loss of islets due to IBMIR. To increase the impact of the observation, allogeneic study is very important to understand if similar results will be observed. In addition, this strategy needs to be tested with other clinically used agents, such as heparin to assess its beneficial impact. Extensive mechanistic studies are 
warranted in autologous as well as allogeneic settings to better understand how SA-CD47 functions that will allow further refinement of this approach and its combinatorial use with agents that may work in synergy for a better outcome. Most importantly, this approach needs to be tested for settings involved cell transplantation, such as HSC or hepatocytes, as a single agent and also in combination with other modulators. In this context, the ProtEx $^{\mathrm{TM}}$ technology is a rather effective platform to display both SA-FasL and SA-CD47 and the implication of these both molecules on HSCT and allogeneic islet transplantation will be important to explore. 


\section{REFERENCES}

1 Welniak, L. A., Blazar, B. R. \& Murphy, W. J. Immunobiology of allogeneic hematopoietic stem cell transplantation. Annual review of immunology 25, 139170, doi:10.1146/annurev.immunol.25.022106.141606 (2007).

2 Zeiser, R. \& Blazar, B. R. Acute graft-versus-host disease-biologic process, prevention, and therapy. New England Journal of Medicine 377, 2167-2179 (2017).

3 Copelan, E. A. Hematopoietic stem-cell transplantation. New England Journal of Medicine 354, 1813-1826 (2006).

4 Swart, J. F. et al. Haematopoietic stem cell transplantation for autoimmune diseases. Nature reviews. Rheumatology 13, 244-256, doi:10.1038/nrrheum.2017.7 (2017).

5 Gyurkocza, B., Rezvani, A. \& Storb, R. F. Allogeneic hematopoietic cell transplantation: the state of the art. Expert review of hematology 3, 285-299 (2010).

6 Singh, A. K. \& McGuirk, J. P. Allogeneic Stem Cell Transplantation: A Historical and Scientific Overview. Cancer Res 76, 6445-6451, doi:10.1158/00085472.Can-16-1311 (2016).

7 Zuber, J. \& Sykes, M. Mechanisms of mixed chimerism-based transplant tolerance. Trends in immunology 38, 829-843 (2017). 
8 Busque, S. et al. Mixed chimerism and acceptance of kidney transplants after immunosuppressive drug withdrawal. Science translational medicine 12 (2020).

9 Sachs, D. H., Kawai, T. \& Sykes, M. Induction of tolerance through mixed chimerism. Cold Spring Harbor perspectives in medicine 4, a015529, doi:10.1101/cshperspect.a015529 (2014).

10 Schoemans, H. M. et al. EBMT-NIH-CIBMTR Task Force position statement on standardized terminology \& guidance for graft-versus-host disease assessment. Bone marrow transplantation 53, 1401-1415 (2018).

11 Filipovich, A. H. et al. National Institutes of Health consensus development project on criteria for clinical trials in chronic graft-versus-host disease: I. Diagnosis and staging working group report. Biology of blood and marrow transplantation 11, 945-956 (2005).

12 Ferrara, J. L., Levine, J. E., Reddy, P. \& Holler, E. Graft-versus-host disease. Lancet (London, England) 373, 1550-1561, doi:10.1016/s0140-6736(09)60237-3 (2009).

13 Zeiser, R. \& Blazar, B. R. Pathophysiology of chronic graft-versus-host disease and therapeutic targets. New England Journal of Medicine 377, 2565-2579 (2017).

14 Billingham, R. E. The biology of graft-versus-host reactions. Harvey lectures $\mathbf{6 2}$, 21-78 (1966).

15 Holtan, S. G., Pasquini, M. \& Weisdorf, D. J. Acute graft-versus-host disease: a bench-to-bedside update. Blood 124, 363-373, doi:10.1182/blood-2014-01514786 (2014). 
16 Jaksch, M. \& Mattsson, J. The pathophysiology of acute graft-versus-host disease. Scandinavian journal of immunology 61, 398-409 (2005).

17 Reddy, P. \& Ferrara, J. L. Immunobiology of acute graft-versus-host disease. Blood reviews 17, 187-194 (2003).

18 Zeiser, R. J. B. j. o. h. Advances in understanding the pathogenesis of graftversus-host disease. (2019).

19 Zeiser, R. J. O. r. \& treatment. Activation of Innate Immunity in Graft-versusHost Disease: Implications for Novel Targets? 38, 239-243 (2015).

20 Jankovic, D. et al. The Nlrp3 inflammasome regulates acute graft-versus-host disease. The Journal of experimental medicine 210, 1899-1910, doi:10.1084/jem.20130084 (2013).

21 Mielcarek, M. et al. Graft-versus-host disease after nonmyeloablative versus conventional hematopoietic stem cell transplantation. Blood 102, 756-762 (2003).

22 Teshima, T. et al. Acute graft-versus-host disease does not require alloantigen expression on host epithelium. Nature medicine 8, 575-581 (2002).

23 Shlomchik, W. D. et al. Prevention of graft versus host disease by inactivation of host antigen-presenting cells. Science 285, 412-415 (1999).

24 Stenger, E. O., Turnquist, H. R., Mapara, M. Y. \& Thomson, A. W. Dendritic cells and regulation of graft-versus-host disease and graft-versus-leukemia activity. Blood, The Journal of the American Society of Hematology 119, 50885103 (2012).

25 Blazar, B. R. et al. CD28/B7 interactions are required for sustaining the graftversus-leukemia effect of delayed post-bone marrow transplantation splenocyte 
infusion in murine recipients of myeloid or lymphoid leukemia cells. The Journal of Immunology 159, 3460-3473 (1997).

26 Blazar, B. R., Taylor, P. A., Panoskaltsis-Mortari, A., Sharpe, A. H. \& Vallera, D. A. Opposing roles of CD28: B7 and CTLA-4: B7 pathways in regulating in vivo alloresponses in murine recipients of MHC disparate T cells. The Journal of Immunology 162, 6368-6377 (1999).

27 Saha, A. et al. Programmed death ligand-1 expression on donor T cells drives graft-versus-host disease lethality. The Journal of clinical investigation 126, $2642-2660$ (2016)

28 Niederwieser, D. et al. Endogenous IFN-gamma during human bone marrow transplantation. Analysis of serum levels of interferon and interferon-dependent secondary messages. Transplantation 50, 620-625 (1990).

29 Refaeli, Y., Van Parijs, L., Alexander, S. I. \& Abbas, A. K. Interferon $\gamma$ is required for activation-induced death of T lymphocytes. The Journal of experimental medicine 196, 999-1005 (2002).

30 Refaeli, Y., Van Parijs, L., London, C. A., Tschopp, J. \& Abbas, A. K. Biochemical mechanisms of IL-2-regulated Fas-mediated T cell apoptosis. Immunity 8, 615-623, doi:10.1016/s1074-7613(00)80566-х (1998).

31 Reddy, P. et al. Interleukin-18 regulates acute graft-versus-host disease by enhancing Fas-mediated donor T cell apoptosis. The Journal of experimental medicine 194, 1433-1440 (2001). 
32 Nestel, F., Price, K. S., Seemayer, T. A. \& Lapp, W. S. Macrophage priming and lipopolysaccharide-triggered release of tumor necrosis factor alpha during graftversus-host disease. The Journal of experimental medicine 175, 405-413 (1992).

33 Hill, G. R. \& Ferrara, J. L. J. B. The primacy of the gastrointestinal tract as a target organ of acute graft-versus-host disease: rationale for the use of cytokine shields in allogeneic bone marrow transplantation. 95, 2754-2759 (2000).

$34 \mathrm{Ju}, \mathrm{S}$. T. et al. Fas(CD95)/FasL interactions required for programmed cell death after T-cell activation. Nature 373, 444-448, doi:10.1038/373444a0 (1995).

35 Krammer, P. H. CD95's deadly mission in the immune system. Nature 407, 789795, doi:10.1038/35037728 (2000).

36 Krammer, P. H. in Advances in immunology Vol. 71 163-210 (Elsevier, 1998).

37 Baker, M. B., Altman, N. H., Podack, E. R. \& Levy, R. B. The role of cellmediated cytotoxicity in acute GVHD after MHC-matched allogeneic bone marrow transplantation in mice. The Journal of experimental medicine 183, 26452656 (1996).

38 Blazar, B. R., Murphy, W. J. \& Abedi, M. J. N. r. i. Advances in graft-versus-host disease biology and therapy. 12, 443-458 (2012).

39 Hildebrandt, G. C. et al. Donor-derived TNF- $\alpha$ regulates pulmonary chemokine expression and the development of idiopathic pneumonia syndrome after allogeneic bone marrow transplantation. Blood 104, 586-593 (2004).

40 Krenger, W. et al. Interferon-gamma suppresses T-cell proliferation to mitogen via the nitric oxide pathway during experimental acute graft-versus-host disease. (1996). 
41 Nestel, F. P., Greene, R. N., Kichian, K., Ponka, P. \& Lapp, W. S. Activation of macrophage cytostatic effector mechanisms during acute graft-versus-host disease: release of intracellular iron and nitric oxide-mediated cytostasis. Blood, The Journal of the American Society of Hematology 96, 1836-1843 (2000).

42 Hamawy, M. M. Molecular actions of calcineurin inhibitors. Drug news \& perspectives 16, 277-282, doi:10.1358/dnp.2003.16.5.829315 (2003).

43 Kanakry, C. G. et al. Aldehyde dehydrogenase expression drives human regulatory T cell resistance to posttransplantation cyclophosphamide. Science translational medicine 5, 211ra157-211ra157 (2013).

44 Barrett, A. J. et al. T cell-depleted bone marrow transplantation and delayed T cell add-back to control acute GVHD and conserve a graft-versus-leukemia effect. Bone marrow transplantation 21, 543-551, doi:10.1038/sj.bmt.1701131 (1998).

45 Saad, A. \& Lamb, L. J. B. m. t. Ex vivo T-cell depletion in allogeneic hematopoietic stem cell transplant: past, present and future. 52, 1241 (2017).

46 Klingebiel, T. \& Bader, P. Delayed lymphocyte infusion in children given SCT. Bone marrow transplantation 41, S23 (2008).

47 Soiffer, R. J. T-cell Depletion to Prevent Graft-versus-Host Disease. Thomas ' Hematopoietic Cell Transplantation: Stem Cell Transplantation 1, 1004-1014 (2015).

48 Godfrey, W. R., Krampf, M. R., Taylor, P. A. \& Blazar, B. R. Ex vivo depletion of alloreactive cells based on CFSE dye dilution, activation antigen selection, and dendritic cell stimulation. Blood 103, 1158-1165, doi:10.1182/blood-2003-041098 (2004). 
49 Amrolia, P. J. et al. Selective depletion of donor alloreactive T cells without loss of antiviral or antileukemic responses. Blood 102, 2292-2299 (2003).

50 Askenasy, N. et al. Depletion of naive lymphocytes with fas ligand ex vivo prevents graft-versus-host disease without impairing T cell support of engraftment or graft-versus-tumor activity. Biology of blood and marrow transplantation : journal of the American Society for Blood and Marrow Transplantation 19, 185 195, doi:10.1016/j.bbmt.2012.10.004 (2013).

51 Bohana-Kashtan, O. et al. Selective reduction of graft-versus-host diseasemediating human T cells by ex vivo treatment with soluble Fas ligand. J Immunol 183, 696-705, doi:10.4049/jimmunol.0800561 (2009).

52 Hartwig, U. F., Robbers, M., Wickenhauser, C. \& Huber, C. Murine acute graftversus-host disease can be prevented by depletion of alloreactive T lymphocytes using activation-induced cell death. Blood 99, 3041-3049, doi:10.1182/blood.v99.8.3041 (2002).

53 Hippen, K. L. et al. Massive ex vivo expansion of human natural regulatory T cells (Tregs) with minimal loss of in vivo functional activity. Science translational medicine 3, 83ra41-83ra41 (2011).

54 Kahanovitz, L., Sluss, P. M. \& Russell, S. J. Type 1 diabetes-a clinical perspective. Point of care 16, 37 (2017).

55 Khawandanah, J. Double or hybrid diabetes: A systematic review on disease prevalence, characteristics and risk factors. Nutrition \& diabetes 9, 1-9 (2019).

56 ALFARO, T. CDC releases 2014 Diabetes Report Card. (2015). 
57 Cooke, D. W. \& Plotnick, L. Type 1 diabetes mellitus in pediatrics. pediatr Rev 29, 374-384 (2008).

58 Chiang, J. L., Kirkman, M. S., Laffel, L. M. \& Peters, A. L. Type 1 diabetes through the life span: a position statement of the American Diabetes Association. Diabetes care 37, 2034-2054 (2014).

59 Atkinson, M. A., Eisenbarth, G. S. \& Michels, A. W. Type 1 diabetes. The Lancet 383, 69-82 (2014).

60 Pociot, F. \& Lernmark, A. Genetic risk factors for type 1 diabetes. The Lancet 387, 2331-2339 (2016).

61 Pociot, F. \& McDermott, M. Genetics of type 1 diabetes mellitus. Genes \& Immunity 3, 235-249 (2002).

62 Van Belle, T. L., Coppieters, K. T. \& Von Herrath, M. G. Type 1 diabetes: etiology, immunology, and therapeutic strategies. Physiological reviews 91, 79118 (2011).

63 Yoon, J., Onodera, T. \& Notkins, A. Virus-induced diabetes mellitus. XV. Beta cell damage and insulin-dependent hyperglycemia in mice infected with coxsackie virus B4. The Journal of experimental medicine 148, 1068-1080 (1978).

64 Foulis, A., Farquharson, M. \& Meager, A. Immunoreactive $\alpha$-interferon in insulin-secreting $\beta$ cells in type 1 diabetes mellitus. The Lancet 330, 1423-1427 (1987). 
65 Kaufman, D. et al. Autoimmunity to two forms of glutamate decarboxylase in insulin-dependent diabetes mellitus. The Journal of clinical investigation 89, 283292 (1992).

66 Christen, U., McGavern, D. B., Luster, A. D., von Herrath, M. G. \& Oldstone, M. B. Among CXCR3 chemokines, IFN- $\gamma$-inducible protein of $10 \mathrm{kDa}(\mathrm{CXC}$ chemokine ligand (CXCL) 10) but not monokine induced by IFN- $\gamma$ (CXCL9) imprints a pattern for the subsequent development of autoimmune disease. The Journal of Immunology 171, 6838-6845 (2003).

67 Roncarolo, M.-G. \& Battaglia, M. Regulatory T-cell immunotherapy for tolerance to self antigens and alloantigens in humans. Nature Reviews Immunology 7, 585598 (2007).

68 Atkinson, M. A., Roep, B. O., Posgai, A., Wheeler, D. C. \& Peakman, M. The challenge of modulating $\beta$-cell autoimmunity in type 1 diabetes. The Lancet Diabetes \& Endocrinology 7, 52-64 (2019).

69 Atkinson, M. A., von Herrath, M., Powers, A. C. \& Clare-Salzler, M. Current concepts on the pathogenesis of type 1 diabetes - considerations for attempts to prevent and reverse the disease. Diabetes care 38, 979-988 (2015).

70 Bertuzzi, F. et al. in Advanced Therapies in Pediatric Endocrinology and Diabetology Vol. 30 14-22 (Karger Publishers, 2016).

71 Nassar, A. et al. Total pancreaticoduodenectomy with autologous islet transplantation 14 years after liver-contained composite visceral transplantation. American Journal of Transplantation 18, 2068-2074 (2018). 
72 Piemonti, L. et al. in Transplantation, Bioengineering, and Regeneration of the Endocrine Pancreas $127-137$ (Elsevier, 2020).

73 Rickels, M. R. \& Robertson, R. P. Pancreatic islet transplantation in humans: recent progress and future directions. Endocrine reviews 40, 631-668 (2019).

74 Anazawa, T., Okajima, H., Masui, T. \& Uemoto, S. Current state and future evolution of pancreatic islet transplantation. Annals of gastroenterological surgery 3, 34-42 (2019).

75 Shapiro, A. J., Pokrywczynska, M. \& Ricordi, C. Clinical pancreatic islet transplantation. Nature Reviews Endocrinology 13, 268 (2017).

76 Delaune, V., Berney, T., Lacotte, S. \& Toso, C. Intraportal islet transplantation: the impact of the liver microenvironment. Transplant International 30, 227-238 (2017).

77 Toso, C. et al. Positron-emission tomography imaging of early events after transplantation of islets of Langerhans. Transplantation 79, 353-355 (2005).

78 Harlan, D. M., Kenyon, N. S., Korsgren, O., Roep, B. O. \& Diabetes, I. o. D. S. J. Current advances and travails in islet transplantation. 58, 2175-2184 (2009).

79 Moberg, L. et al. Production of tissue factor by pancreatic islet cells as a trigger of detrimental thrombotic reactions in clinical islet transplantation. The Lancet 360, 2039-2045 (2002).

80 Johansson, H. Mechanisms and Therapeutic Interventions of Instant BloodMediated Inflammatory Reaction (IBMIR), Acta Universitatis Upsaliensis, (2007). 
81 Nilsson, B., Ekdahl, K. N. \& Korsgren, O. Control of instant blood-mediated inflammatory reaction to improve islets of Langerhans engraftment. Curr Opin Organ Transplant 16, 620-626, doi:10.1097/MOT.0b013e32834c2393 (2011).

82 Nilsson, B. J. X. The instant blood-mediated inflammatory reaction in xenogeneic islet transplantation. $\mathbf{1 5}, 96-98$ (2008).

83 Johansson, H. et al. Tissue Factor Produced by the Endocrine Cells of the Islets of Langerhans Is Associated With a Negative Outcome of Clinical Islet Transplantation. Diabetes 54, 1755-1762, doi:10.2337/diabetes.54.6.1755 (2005).

84 Moberg, L. The Role of Innate Immunity in Islet Transplantation: Clinical and Experimental Studies, Acta Universitatis Upsaliensis, (2004).

85 Kanak, M., Saravanan, P. \& Levy, M. Inflammatory response and its impact on outcome of islet transplantation. CellR4 7, e2739 (2019).

86 Kanak, M. A. et al. Inflammatory response in islet transplantation. 2014 (2014).

87 Moberg, L., Korsgren, O. \& Nilsson, B. Neutrophilic granulocytes are the predominant cell type infiltrating pancreatic islets in contact with ABOcompatible blood. Clinical \& Experimental Immunology 142, 125-131 (2005).

88 Moberg, L. et al. Nicotinamide inhibits tissue factor expression in isolated human pancreatic islets: implications for clinical islet transplantation1. Transplantation 76, 1285-1288 (2003).

89 Bennet, W., Groth, C.-G., Larsson, R., Nilsson, B. \& Korsgren, O. J. U. j. o. m. s. Isolated human islets trigger an instant blood mediated inflammatory reaction: implications for intraportal islet transplantation as a treatment for patients with type 1 diabetes. 105, 125-133 (2000). 
90 Cabric, S. et al. Islet surface heparinization prevents the instant blood-mediated inflammatory reaction in islet transplantation. Diabetes 56, 2008-2015 (2007).

91 Johansson, H. et al. Low molecular weight dextran sulfate: a strong candidate drug to block IBMIR in clinical islet transplantation. Am J Transplant 6, 305-312, doi:10.1111/j.1600-6143.2005.01186.x (2006).

92 Akima, S. et al. Tirofiban and activated protein C synergistically inhibit the instant blood mediated inflammatory reaction (IBMIR) from allogeneic islet cells exposure to human blood. 9, 1533-1540 (2009).

93 Contreras, J. L. et al. Activated protein C preserves functional islet mass after intraportal transplantation: a novel link between endothelial cell activation, thrombosis, inflammation, and islet cell death. Diabetes 53, 2804-2814 (2004).

94 Bozkurt, N. C. et al. Hepatic hematoma after islet cell transplantation. Transplantation 95, e73-76, doi:10.1097/TP.0b013e31829468e2 (2013).

95 Hirshberg, B. et al. Benefits and risks of solitary islet transplantation for type 1 diabetes using steroid-sparing immunosuppression: the National Institutes of Health experience. Diabetes care 26, 3288-3295, doi:10.2337/diacare.26.12.3288 (2003).

96 Kuraya, D. et al. Efficacy of DHMEQ, a NF- $\kappa$ B inhibitor, in islet transplantation: I. HMGB1 suppression by DHMEQ prevents early islet graft damage. Transplantation 96, 445-453 (2013).

97 Wang, J. et al. alpha-1 antitrypsin enhances islet engraftment by suppression of instant blood-mediated inflammatory reaction. Diabetes, db161036 (2017). 
98 Citro, A. et al. CCL2/MCP-1 and CXCL12/SDF-1 blockade by L-aptamers improve pancreatic islet engraftment and survival in mouse. Am J Transplant, doi:10.1111/ajt.15518 (2019).

99 Brown, E. J. \& Frazier, W. A. Integrin-associated protein (CD47) and its ligands. Trends in cell biology 11, 130-135 (2001).

100 Barclay, A. N. Signal regulatory protein alpha (SIRP $\alpha) / C D 47$ interaction and function. Current opinion in immunology 21, 47-52 (2009).

101 Oldenborg, P.-A. CD47: a cell surface glycoprotein which regulates multiple functions of hematopoietic cells in health and disease. ISRN hematology 2013 (2013).

102 Brown, E. Integrin-associated protein (CD47): an unusual activator of G protein signaling. The Journal of clinical investigation 107, 1499-1500 (2001).

103 Barclay, A. N. \& Van den Berg, T. K. The interaction between signal regulatory protein alpha $(\mathrm{SIRP} \alpha)$ and $\mathrm{CD} 47$ : structure, function, and therapeutic target. Annual review of immunology 32, 25-50 (2014).

104 Wiersma, V. R., van Bommel, P. E., De Bruyn, M., Helfrich, W. \& Bremer, E. CD47, a multi-facetted target for cancer immunotherapy. Atlas of Genetics and Cytogenetics in Oncology and Haematology (2015).

105 Soto-Pantoja, D. R. et al. Therapeutic opportunities for targeting the ubiquitous cell surface receptor CD47. Expert opinion on therapeutic targets 17, 89-103 (2013). 
106 Advani, R. et al. CD47 Blockade by Hu5F9-G4 and Rituximab in Non-Hodgkin's Lymphoma. The New England journal of medicine 379, 1711-1721, doi:10.1056/NEJMoa1807315 (2018).

107 Barrera, L. et al. CD47 overexpression is associated with decreased neutrophil apoptosis/phagocytosis and poor prognosis in non-small-cell lung cancer patients. British journal of cancer 117, 385-397, doi:10.1038/bjc.2017.173 (2017).

108 Blazar, B. R. et al. CD47 (integrin-associated protein) engagement of dendritic cell and macrophage counterreceptors is required to prevent the clearance of donor lymphohematopoietic cells. The Journal of experimental medicine 194, 541-549, doi:10.1084/jem.194.4.541 (2001).

109 Sick, E. et al. CD47 update: a multifaceted actor in the tumour microenvironment of potential therapeutic interest. 167, 1415-1430 (2012).

110 Oldenborg, P. A. et al. Role of CD47 as a marker of self on red blood cells. Science 288, 2051-2054, doi:8601 [pii] (2000).

111 Oldenborg, P.-A., Gresham, H. D. \& Lindberg, F. P. Cd47-signal regulatory protein $\alpha(\operatorname{Sirp} \alpha)$ regulates $\mathrm{Fc} \gamma$ and complement receptor-mediated phagocytosis. Journal of Experimental Medicine 193, 855-862 (2001).

112 Jaiswal, S. et al. CD47 is upregulated on circulating hematopoietic stem cells and leukemia cells to avoid phagocytosis. Cell 138, 271-285 (2009).

113 Majeti, R. et al. CD47 is an adverse prognostic factor and therapeutic antibody target on human acute myeloid leukemia stem cells. Cell 138, 286-299, doi:10.1016/j.cell.2009.05.045 (2009). 
114 Van, V. Q. et al. Expression of the self-marker CD47 on dendritic cells governs their trafficking to secondary lymphoid organs. The EMBO journal $\mathbf{2 5}, 5560-5568$ (2006).

115 Hagnerud, S. et al. Deficit of CD47 results in a defect of marginal zone dendritic cells, blunted immune response to particulate antigen and impairment of skin dendritic cell migration. The Journal of Immunology 176, 5772-5778 (2006).

116 Finley, M. J. et al. Diminished adhesion and activation of platelets and neutrophils with CD47 functionalized blood contacting surfaces. Biomaterials 33, 5803-5811 (2012).

117 Stachelek, S. J. et al. The effect of CD47 modified polymer surfaces on inflammatory cell attachment and activation. Biomaterials 32, 4317-4326 (2011).

118 Matlung, H. L. et al. Neutrophils Kill Antibody-Opsonized Cancer Cells by Trogoptosis. 23, 3946-3959. e3946 (2018).

119 Navarro-Alvarez, N. \& Yang, Y.-G. CD47: a new player in phagocytosis and xenograft rejection. Cellular \& molecular immunology 8, 285 (2011).

120 Navarro-Alvarez, N. \& Yang, Y. G. Lack of CD47 on donor hepatocytes promotes innate immune cell activation and graft loss: a potential barrier to hepatocyte xenotransplantation. Cell Transplant 23, 345-354, doi:10.3727/096368913x663604 (2014).

121 Ide, K. et al. Role for CD47-SIRP $\alpha$ signaling in xenograft rejection by macrophages. 104, 5062-5066 (2007).

122 Takenaka, K. et al. Polymorphism in Sirpa modulates engraftment of human hematopoietic stem cells. Nature immunology 8, 1313-1323 (2007). 
123 Faustman, D. L. in Principles of Tissue Engineering 531-542 (Elsevier, 2014).

124 Gea-Banacloche, J. C. in Principles of molecular medicine 893-904 (Springer, 2006).

125 Bluestone, J. A. \& Bour-Jordan, H. Current and future immunomodulation strategies to restore tolerance in autoimmune diseases. Cold Spring Harbor perspectives in biology 4, a007542 (2012).

126 Singh, N. P., Yolcu, E. S., Askenasy, N. \& Shirwan, H. ProtEx: a novel technology to display exogenous proteins on the cell surface for immunomodulation. Annals of the New York Academy of Sciences 1056, 344-358, doi:10.1196/annals.1352.036 (2005).

127 Schabowsky, R. H. et al. ProtEx technology for the generation of novel therapeutic cancer vaccines. Exp Mol Pathol 86, 198-207, doi:10.1016/j.yexmp.2009.01.010 (2009).

128 Headen, D. M. et al. Local immunomodulation with Fas ligand-engineered biomaterials achieves allogeneic islet graft acceptance. Nature materials, 1 (2018).

129 Yolcu, E. S., Askenasy, N., Singh, N. P., Cherradi, S. E. \& Shirwan, H. Cell membrane modification for rapid display of proteins as a novel means of immunomodulation: FasL-decorated cells prevent islet graft rejection. Immunity 17, 795-808, doi:Doi 10.1016/S1074-7613(02)00482-X (2002).

130 Yolcu, E. S. et al. Pancreatic islets engineered with SA-FasL protein establish robust localized tolerance by inducing regulatory $\mathrm{T}$ cells in mice. The Journal of Immunology, 1003266 (2011). 
131 Yolcu, E. S. et al. Induction of tolerance to cardiac allografts using donor splenocytes engineered to display on their surface an exogenous Fas Ligand protein. J Immunol 181, 931-939, doi:DOI 10.4049/jimmunol.181.2.931 (2008).

132 Olson, C. A., Williams, L. C., McLaughlin-Taylor, E. \& McMillan, M. Creation of $\mathrm{H}-2$ class I epitopes using synthetic peptides: recognition by alloreactive cytotoxic T lymphocytes. Proc. Natl. Acad. Sci. USA 86, 1031-1035 (1989).

133 Pearl-Yafe, M. et al. Expression of Fas and Fas-ligand in donor hematopoietic stem and progenitor cells is dissociated from the sensitivity to apoptosis. Experimental hematology 35, 1601-1612 (2007).

134 Sharma, R. K. et al. 4-1BB ligand as an effective multifunctional immunomodulator and antigen delivery vehicle for the development of therapeutic cancer vaccines. Cancer research, 0008-5472. CAN-0009-4480 (2010).

135 Sharma, R. K. et al. SA-4-1BBL as the immunomodulatory component of a HPV$16 \mathrm{E} 7$ protein based vaccine shows robust therapeutic efficacy in a mouse cervical cancer model. Vaccine 28, 5794-5802 (2010).

136 Lenardo, M. et al. Mature T lymphocyte apoptosis-immune regulation in a dynamic and unpredictable antigenic environment. Annual review of immunology 17, 221-253 (1999).

137 Siegel, R. M., Chan, F. K., Chun, H. J. \& Lenardo, M. J. The multifaceted role of Fas signaling in immune cell homeostasis and autoimmunity. Nature immunology 1, 469-474, doi:10.1038/82712 (2000). 
138 Lenardo, M. J. Interleukin-2 programs mouse $\alpha \beta$ T lymphocytes for apoptosis. Nature 353, 858 (1991).

139 Georgantas, R. W., 3rd, Bohana-Kashtan, O. \& Civin, C. I. Ex vivo soluble fas ligand treatment of donor cells to selectively reduce murine acute graft versus host disease. Transplantation 82, 471-478, doi:10.1097/01.tp.0000229435.58898.c5 (2006).

140 Dybedal, I. et al. Human reconstituting hematopoietic stem cells up-regulate Fas expression upon active cell cycling but remain resistant to Fas-induced suppression. Blood 102, 118-126 (2003).

141 Fukuda, S. \& Pelus, L. M. Regulation of the inhibitor-of-apoptosis family member survivin in normal cord blood and bone marrow CD34+ cells by hematopoietic growth factors: implication of survivin expression in normal hematopoiesis. Blood 98, 2091-2100 (2001).

142 Fukuda, S. \& Pelus, L. M. Elevation of Survivin levels by hematopoietic growth factors occurs in quiescent CD34+ hematopoietic stem and progenitor cells before cell cycle entry. Cell cycle 1, 323-324 (2002).

143 Pearl-Yafe, M. et al. Fas ligand enhances hematopoietic cell engraftment through abrogation of alloimmune responses and nonimmunogenic interactions. Stem Cells 25, 1448-1455 (2007).

144 Fritzsching, B. et al. In contrast to effector T cells, CD4+CD25+FoxP3+ regulatory T cells are highly susceptible to CD95 ligand- but not to TCRmediated cell death. J Immunol 175, 32-36, doi:10.4049/jimmunol.175.1.32 (2005). 
145 Yolcu, E. S., Askenasy, N., Singh, N. P., Cherradi, S.-E. L. \& Shirwan, H. Cell membrane modification for rapid display of proteins as a novel means of immunomodulation: FasL-decorated cells prevent islet graft rejection. Immunity 17, 795-808 (2002).

146 Kendal, A. R. et al. Sustained suppression by Foxp3+ regulatory T cells is vital for infectious transplantation tolerance. Journal of Experimental Medicine 208, 2043-2053 (2011).

147 Brennan, T. V. et al. A new T-cell receptor transgenic model of the CD4+ direct pathway: level of priming determines acute versus chronic rejection.

Transplantation 85, 247-255, doi:10.1097/TP.0b013e31815e883e (2008).

148 King, M. A. et al. Human peripheral blood leucocyte non-obese diabetic-severe combined immunodeficiency interleukin-2 receptor gamma chain gene mouse model of xenogeneic graft-versus-host-like disease and the role of host major histocompatibility complex. Clinical and experimental immunology 157, 104-118, doi:10.1111/j.1365-2249.2009.03933.x (2009).

149 Tugues, S. et al. Graft-versus-host disease, but not graft-versus-leukemia immunity, is mediated by GM-CSF-licensed myeloid cells. 10, eaat8410 (2018).

150 Cooke, K. R. et al. An experimental model of idiopathic pneumonia syndrome after bone marrow transplantation: I. The roles of minor $\mathrm{H}$ antigens and endotoxin. (1996).

151 Pakyari, M. et al. A new method for skin grafting in murine model. Wound Repair and Regeneration 24, 695-704 (2016). 
152 Ehx, G. et al. Xenogeneic Graft-Versus-Host Disease in Humanized NSG and NSG-HLA-A2/HHD Mice. Frontiers in Immunology 9 , doi:10.3389/fimmu.2018.01943 (2018).

153 Gendelman, M. et al. Selective elimination of alloreactive donor T cells attenuates graft-versus-host disease and enhances T-cell reconstitution. 9, 742-752 (2003).

154 Henden, A. S. \& Hill, G. R. Cytokines in graft-versus-host disease. The Journal of Immunology 194, 4604-4612 (2015).

$155 \mathrm{Fu}$, J. et al. T-bet is critical for the development of acute graft-versus-host disease through controlling T cell differentiation and function. J Immunol 194, 388-397, doi:10.4049/jimmunol.1401618 (2015).

156 Zheng, W. \& Flavell, R. A. The transcription factor GATA-3 is necessary and sufficient for Th2 cytokine gene expression in CD4 T cells. Cell 89, 587-596, doi:10.1016/s0092-8674(00)80240-8 (1997).

157 Edinger, M. et al. CD4+ CD25+ regulatory T cells preserve graft-versus-tumor activity while inhibiting graft-versus-host disease after bone marrow transplantation. Nature medicine 9, 1144-1150 (2003).

158 Hoffmann, P., Ermann, J., Edinger, M., Fathman, C. G. \& Strober, S. Donor-type CD4+ CD25+ regulatory T cells suppress lethal acute graft-versus-host disease after allogeneic bone marrow transplantation. The Journal of experimental medicine 196, 389-399 (2002).

159 Askenasy, N., Yolcu, E. S., Yaniv, I. \& Shirwan, H. Induction of tolerance using Fas ligand: a double-edged immunomodulator. Blood 105, 1396-1404, doi:10.1182/blood-2004-06-2364 (2005). 
160 Green, D. R., Droin, N. \& Pinkoski, M. Activation-induced cell death in T cells. Immunol Rev 193, 70-81, doi:10.1034/j.1600-065x.2003.00051.x (2003).

161 Morgan, M. J., Kim, Y.-S. \& Liu, Z.-g. Membrane-bound Fas ligand requires RIP1 for efficient activation of caspase- 8 within the death-inducing signaling complex. The Journal of Immunology 183, 3278-3284 (2009).

162 Riegel, C. et al. Efficient treatment of murine acute GvHD by in vitro expanded donor regulatory T cells. Leukemia, 1-14 (2019).

163 Beilhack, A. et al. In vivo analyses of early events in acute graft-versus-host disease reveal sequential infiltration of T-cell subsets. Blood 106, 1113-1122, doi:10.1182/blood-2005-02-0509 (2005).

164 Buxbaum, N. P. et al. In vivo kinetics and nonradioactive imaging of rapidly proliferating cells in graft-versus-host disease. JCI insight $\mathbf{2}$, doi:10.1172/jci.insight.92851 (2017).

165 Chen, B. J. et al. Inability of memory T cells to induce graft-versus-host disease is a result of an abortive alloresponse. Blood 109, 3115-3123, doi:10.1182/blood2006-04-016410 (2007).

166 Ermann, J. et al. Only the CD62L+ subpopulation of CD4+CD25+ regulatory T cells protects from lethal acute GVHD. Blood 105, 2220-2226, doi:10.1182/blood-2004-05-2044 (2005).

167 Nguyen, V. H. et al. In vivo dynamics of regulatory T-cell trafficking and survival predict effective strategies to control graft-versus-host disease following allogeneic transplantation. Blood 109, 2649-2656 (2007). 
168 Overacre-Delgoffe, A. E. et al. Interferon- $\gamma$ drives Treg fragility to promote antitumor immunity. Cell 169, 1130-1141. e1111 (2017).

169 Ohkura, N., Kitagawa, Y. \& Sakaguchi, S. Development and maintenance of regulatory T cells. Immunity 38, 414-423 (2013).

170 Krenger, W., Snyder, K. M., Byon, J., Falzarano, G. \& Ferrara, J. Polarized type 2 alloreactive $\mathrm{CD} 4+$ and $\mathrm{CD} 8+$ donor $\mathrm{T}$ cells fail to induce experimental acute graft-versus-host disease. The Journal of Immunology 155, 585-593 (1995).

171 Chakraborty, R. et al. Robust and cost effective expansion of human regulatory T cells highly functional in a xenograft model of graft-versus-host disease.

Haematologica 98, 533-537 (2013).

172 Ganguly, S. et al. Donor CD4+ Foxp3+ regulatory T cells are necessary for posttransplantation cyclophosphamide-mediated protection against GVHD in mice. Blood 124, 2131-2141, doi:10.1182/blood-2013-10-525873 (2014).

173 Naziruddin, B. et al. Evidence for instant blood-mediated inflammatory reaction in clinical autologous islet transplantation. American Journal of Transplantation 14, 428-437 (2014).

174 Bennet, W. et al. Incompatibility between human blood and isolated islets of Langerhans: a finding with implications for clinical intraportal islet transplantation? Diabetes 48, 1907-1914 (1999).

175 Korsgren, O. et al. Current status of clinical islet transplantation. Transplantation 79, 1289-1293, doi:00007890-200505270-00003 [pii] (2005). 
176 Eich, T., Eriksson, O. \& Lundgren, T. Visualization of early engraftment in clinical islet transplantation by positron-emission tomography. N. Engl. J. Med 356, 2754-2755, doi:356/26/2754 [pii];10.1056/NEJMc070201 [doi] (2007).

177 Anquetil, F. et al. Alpha cells, the main source of IL-1beta in human pancreas. J Autoimmun 81, 68-73, doi:10.1016/j.jaut.2017.03.006 (2017).

178 Negi, S. et al. Analysis of beta-cell gene expression reveals inflammatory signaling and evidence of dedifferentiation following human islet isolation and culture. PLoS One 7, e30415, doi:10.1371/journal.pone.0030415 (2012).

179 Chinnakotla, S. et al. Total pancreatectomy and islet autotransplantation in children for chronic pancreatitis: indication, surgical techniques, postoperative management, and long-term outcomes. Annals of surgery 260, 56-64, doi:10.1097/sla.0000000000000569 (2014).

180 Bramis, K. et al. Systematic review of total pancreatectomy and islet autotransplantation for chronic pancreatitis. The British journal of surgery $\mathbf{9 9}$, 761-766, doi:10.1002/bjs.8713 (2012).

181 Chhabra, A. et al. Hematopoietic stem cell transplantation in immunocompetent hosts without radiation or chemotherapy. 8, 351ra105-351ra105 (2016).

182 Zhao, X. Targeting CD47-SIRP interactions for potentiating therapeutic antibodymediated tumor cell destruction by phagocytes.

183 Wang, H. et al. Attenuation of phagocytosis of xenogeneic cells by manipulating CD47. Blood 109, 836-842 (2007).

184 Demeure, C. et al. CD47 engagement inhibits cytokine production and maturation of human dendritic cells. The Journal of Immunology 164, 2193-2199 (2000). 
185 Zhang, M. et al. Donor CD47 controls T cell alloresponses and is required for tolerance induction following hepatocyte allotransplantation. 6, 26839 (2016).

186 Feng, M. et al. Phagocytosis checkpoints as new targets for cancer immunotherapy. Nat Rev Cancer 19, 568-586, doi:10.1038/s41568-019-0183-z (2019).

187 Willingham, S. B. et al. The CD47-signal regulatory protein alpha (SIRPa) interaction is a therapeutic target for human solid tumors. Proc Natl Acad Sci U S A 109, 6662-6667, doi:10.1073/pnas.1121623109 (2012).

188 Iribarren, K. et al. Anticancer effects of anti-CD47 immunotherapy in vivo. Oncoimmunology 8, 1550619, doi:10.1080/2162402x.2018.1550619 (2019).

189 Chao, M. P. et al. Therapeutic antibody targeting of CD47 eliminates human acute lymphoblastic leukemia. Cancer Res 71, 1374-1384, doi:10.1158/00085472.Can-10-2238 (2011).

190 Dai, H. et al. Donor SIRP $\alpha$ polymorphism modulates the innate immune response to allogeneic grafts. Science immunology 2 (2017).

191 Dai, H. et al. Donor SIRPalpha polymorphism modulates the innate immune response to allogeneic grafts. Sci Immunol 2, doi:10.1126/sciimmunol.aam6202 (2017).

192 Teraoka, Y., Ide, K., Morimoto, H., Tahara, H. \& Ohdan, H. J. P. o. Expression of recipient CD47 on rat insulinoma cell xenografts prevents macrophage-mediated rejection through SIRP $\alpha$ inhibitory signaling in mice. 8, e58359 (2013). 
193 Leung, C. S., Li, J., Xu, F., Wong, A. S. \& Lui, K. O. J. T. F. J. Ectopic expression of recipient CD47 inhibits mouse macrophage-mediated immune rejection against human stem cell transplants. fj. 201800449R (2018).

194 Takenaka, K. et al. Polymorphism in Sirpa modulates engraftment of human hematopoietic stem cells. Nat. Immunol 8, 1313-1323, doi:ni1527 [pii];10.1038/ni1527 [doi] (2007).

195 Ide, K. et al. Role for CD47-SIRPalpha signaling in xenograft rejection by macrophages. Proc. Natl. Acad. Sci. U. S. A 104, 5062-5066, doi:0609661104 [pii];10.1073/pnas.0609661104 [doi] (2007).

196 Tena, A. et al. Transgenic expression of human CD47 markedly increases engraftment in a murine model of pig-to-human hematopoietic cell transplantation. Am. J. Transplant 14, 2713-2722, doi:10.1111/ajt.12918 [doi] (2014).

197 Muthyala, S. et al. The effect of hypoxia on free and encapsulated adult porcine islets-an in vitro study. Xenotransplantation 24, doi:10.1111/xen.12275 (2017).

198 Blom, K. G. et al. Isolation of murine intrahepatic immune cells employing a modified procedure for mechanical disruption and functional characterization of the B, T and natural killer T cells obtained. Clinical \& Experimental Immunology 155, 320-329 (2009).

199 Badet, L. et al. The interaction between primate blood and mouse islets induces accelerated clotting with islet destruction. Xenotransplantation 9, 91-96 (2002). 
200 Singh, N. P. et al. A novel approach to cancer immunotherapy: tumor cells decorated with CD80 generate effective antitumor immunity. Cancer Res $\mathbf{6 3}$, 4067-4073 (2003).

201 Yolcu, E. S. et al. Induction of tolerance to cardiac allografts using donor splenocytes engineered to display on their surface an exogenous fas ligand protein. J. Immunol 181, 931-939, doi:181/2/931 [pii] (2008).

202 Sharma, R. K., Yolcu, E. S., Elpek, K. G. \& Shirwan, H. Tumor cells engineered to codisplay on their surface 4-1BBL and LIGHT costimulatory proteins as a novel vaccine approach for cancer immunotherapy. Cancer Gene Ther 17, 730741, doi:10.1038/cgt.2010.29 (2010).

203 Kaminitz, A. et al. Killer Treg restore immune homeostasis and suppress autoimmune diabetes in prediabetic NOD mice. J. Autoimmun 37, 39-47, doi:S0896-8411(11)00031-X [pii];10.1016/j.jaut.2011.03.003 [doi] (2011).

204 Yolcu, E. S. et al. Pancreatic islets engineered with SA-FasL protein establish robust localized tolerance by inducing regulatory T cells in mice. J. Immunol 187, 5901-5909, doi:jimmunol.1003266 [pii];10.4049/jimmunol.1003266 [doi] (2011).

205 Tsai, R. K. \& Discher, D. E. Inhibition of "self" engulfment through deactivation of myosin-II at the phagocytic synapse between human cells. The Journal of cell biology 180, 989-1003 (2008).

206 Sano, T., Vajda, S., Smith, C. L. \& Cantor, C. R. Engineering subunit association of multisubunit proteins: a dimeric streptavidin. Proc Natl. Acad. Sci. U. S. A 94, 6153-6158 (1997). 
207 Woodward, K. B. et al. Pancreatic islets engineered with a FasL protein induce systemic tolerance at the induction phase that evolves into long-term graftlocalized immune privilege. Am J Transplant, doi:10.1111/ajt.15747 (2019).

208 Jaiswal, S. et al. CD47 is upregulated on circulating hematopoietic stem cells and leukemia cells to avoid phagocytosis. Cell 138, 271-285, doi:S00928674(09)00651-5 [pii];10.1016/j.cell.2009.05.046 [doi] (2009).

209 Waern, J. M. et al. Ectopic expression of murine CD47 minimizes macrophage rejection of human hepatocyte xenografts in immunodeficient mice. Hepatology 56, 1479-1488, doi:10.1002/hep.25816 (2012).

210 Matsuoka, N. et al. High-mobility group box 1 is involved in the initial events of early loss of transplanted islets in mice. The Journal of clinical investigation 120, 735-743 (2010).

211 Cui, W. et al. Thrombomodulin Improves Early Outcomes After Intraportal Islet Transplantation. 9, 1308-1316, doi:10.1111/j.1600-6143.2009.02652.x (2009).

212 Cheng, F. et al. A critical role for Stat3 signaling in immune tolerance. Immunity 19, 425-436 (2003).

213 Toledano, N., Gur-Wahnon, D., Ben-Yehuda, A. \& Rachmilewitz, J. Novel CD47: SIRPalpha dependent mechanism for the activation of STAT3 in antigenpresenting cell. PLoS One 8, e75595, doi:10.1371/journal.pone.0075595 (2013).

214 Latour, S. et al. Bidirectional negative regulation of human $\mathrm{T}$ and dendritic cells by CD47 and its cognate receptor signal-regulator protein-alpha: down-regulation of IL-12 responsiveness and inhibition of dendritic cell activation. J. Immunol 167, 2547-2554 (2001). 
215 Wang, Y., Wang, H., Bronson, R., Fu, Y. \& Yang, Y. G. Rapid dendritic cell activation and resistance to allotolerance induction in anti-CD154-treated mice receiving CD47-deficient donor-specific transfusion. Cell Transplant 23, 355363, doi:ct0662wang [pii];10.3727/096368912X661346 [doi] (2014).

216 Zhang, M. et al. Donor CD47 controls T cell alloresponses and is required for tolerance induction following hepatocyte allotransplantation. Scientific reports $\mathbf{6}$, 26839, doi:10.1038/srep26839 (2016).

217 Pengam, S. et al. SIRPalpha/CD47 axis controls the maintenance of transplant tolerance sustained by myeloid-derived suppressor cells. Am J Transplant 19, 3263-3275, doi:10.1111/ajt.15497 (2019).

218 Skoumal, M. et al. Localized immune tolerance from FasL-functionalized PLG scaffolds. Biomaterials 192, 271-281, doi:10.1016/j.biomaterials.2018.11.015 (2019).

219 Headen, D. M. et al. Local immunomodulation Fas ligand-engineered biomaterials achieves allogeneic islet graft acceptance. Nat Mater 17, 732-739, doi:10.1038/s41563-018-0099-0 (2018).

220 Yolcu, E. S. et al. Induction of tolerance to cardiac allografts using donor splenocytes engineered to display on their surface an exogenous fas ligand protein. The Journal of Immunology 181, 931-939 (2008). 


\title{
CURRICULUM VITAE
}

\author{
Pradeep Shrestha
}

580 S. Preston St. Rm.117

(502)-807-9426 (cell)

Baxter II, Louisville, KY, 40202

USA

\section{EDUCATION}

Doctor of Philosophy in Microbiology and Immunology

April, 2020

University of Louisville; Louisville, KY

Master of Science in Microbiology and Immunology

Aug, 2017

University of Louisville; Louisville, KY

Master of Science in Biotechnology

Jan, 2014

Tribhuvan University; Nepal

Bachelor of Science in Microbiology

Dec, 2009

Tribhuvan University; Nepal

\section{RESEARCH EXPERIENCE}

Graduate researcher at University of Louisville

2016-present

Department of Microbiology and Immunology

Mentors: Dr Haval Shirwan and Dr. Esma S. Yolcu

Dissertation project

Title: Targeting FasL-engineered donor cells as strategy to prevent acute Graft-versushost disease (GVHD).

We employed a novel strategy to eliminate alloreactive cells to prevent acute GVHD. Using haploidentical and xenogeneic settings we show that, engineering the donor cells with novel form of FasL, can significantly eliminate pathogenic alloreactive $\mathrm{T}$ cells, thus successfully preventing lethal GVHD. 


\section{Side projects}

Title: Localized immunotolerance in islet transplantation setting by employing FasL engineered microgels.

The goal of the project was to establish localized tolerance for islet grafts in allogeneic transplantation settings under transient cover of immunosuppression. We showed that targeting alloreactive cells by FasL-engineered microgels and short-term treatment with rapamycin is efficient enough to establish localized tolerance in murine model. The study resulted in one co-first author paper published in Nature Materials (2018). Pre-clinical study in non-human primate model is ongoing with collaboration to Harvard Medical school and Georgia Tech university.

Title: Novel form of CD137 as cancer immunoprevention agent in multiple murine cancer models.

The goal of the project was to test if novel form of CD137 (SA-4-1BBL) can prevent tumor development in murine model. We showed that animals treated with SA-4-1BBL, when challenged with cancer cells, are protected from cancer development. The study was published in Cancer Research journal (2019).

Title: Modification of extracellular vesicles as a strategy for nanotherapeutics.

The ongoing project is in collaboration with Carnegie Mellon group where extracellular vesicles can be effectively engineered with desired proteins and can be established as nanotherapeutics. As proof of concept, FasL-displaying extracellular vesicles can significantly eliminated alloreactive cells which can be employed in autoimmune disease models. This study has, so far, been published in ACS Nano (2019).

Graduate research at Tribhuvan University

2012-2013

Central department of Biotechnology

Mentor: Dr. Deepak Raj Pant

- In vitro axillary proliferation and propagation of Swertia Chirayita, a medicinal plant. This research work was supported by Thesis support grant, UGC, Nepal.

- Phytochemical evaluation of different medicinal plants. This research work was granted as faculty research grant to Dr. Deepak Raj Pant as primary investigator by UGC, Nepal. I served as an associate researcher for the project.

\section{AWARDS}

- Travel award, AAI (2018).

- Graduate Fellowship (2015-2017), Integrated Programs in Biomedical Sciences, University of Louisville, Louisville, USA.

- Korean Government Scholarship Program (2014)

- Dissertation Grant, University Grant Commissions, Bhaktapur, Nepal (2012-2013). 


\section{MEMBERSHIPS}

- American Association for the Advancement of Sciences (AAAS)

- American Association of Immunologists

\section{PROFESSIONAL APPOINTMENTS/ RESPONSIBILITIES}

- Trained and mentored several people including high school students, rotating graduate students and postdoctoral fellow in the lab.

- Lecturer at Kantipur Valley College, Kathmandu, Nepal (2014-2015)

\section{PROFESSIONAL IDENTIFIER}

Google scholar link: https://scholar.google.com/citations?user=LIEcvlsAAAAJ\&hl=en

ORCID identifier: 0000-0003-3418-6790

\section{PUBLICATIONS}

- Pradeep Shrestha, Lalit Batra, Tariq Malik, Min Tan, Orlando Grimany-Nuno, Esma. S. Yolcu, Haval Shirwan. Immune checkpoint CD47 molecule engineered islets mitigate instant blood-mediated inflammatory reaction and show improved engraftment following intraportal transplantation. (Resubmitted with minor revision, American Journal of Transplantation)

- Lalit Batra*, Pradeep Shrestha*, Hong Zhao, Kyle B. Woodward, Min Tan, Orlando Grimany-Nuno, Alper Togay, Maria M. Coronel, Andrés J. García, Haval Shirwan, Esma S. Yolcu. Localized immunomodulation with PD-L1 results in sustained survival and function of allogeneic islets without chronic immunosuppression. (In Press Journal of Immunology)

- Woodward KB*, Zhao H*, Shrestha P, Batra L, Tan M, Grimany-Nuno O, Bandura-Morgan L, Askenasy N, Shirwan H, Yolcu ES. Pancreatic islets engineered with a FasL protein induce systemic tolerance at the induction phase that evolves into long-term graft-localized immune privilege. American Journal of Transplantation. 2019 Dec 18.

- Yerneni SS*, Lathwal S*, Shrestha P., Shirwan H, Matyjaszewski K, Weiss L, Yolcu ES, Campbell PG, Das SR. Rapid On-Demand Extracellular Vesicle Augmentation with Versatile Oligonucleotide Tethers. ACS nano. 2019 Aug 22;13(9):10555-65. 
- Barsoumian HB*, Batra L*, Shrestha P., Bowen WS, Zhao H, Egilmez NK, Gomez-Gutierrez JG, Yolcu ES, Shirwan H. A Novel Form of 4-1BBL Prevents Cancer Development via Nonspecific Activation of CD4+ T and Natural Killer Cells. Cancer research. 2019 Feb 15;79(4):783-94.

- Headen DM*, Woodward KB*, Coronel MM*, Shrestha P. ${ }^{*}$, Weaver JD, Zhao H, Tan M, Hunckler MD, Bowen WS, Johnson CT, Shea L. Local immunomodulation with Fas ligand-engineered biomaterials achieves allogeneic islet graft acceptance. Nature materials. 2018 Aug;17(8):732.

- Williams, C. L., Yolcu,E. S., Woodward, K. B., Zhao, H., Bowen, W. S., Shrestha, P., Batra, L., Askenasy, N. and Shirwan, H. FasL mediated apoptosis as an effective means of inducing tolerance to allogenic pancreatic islets. Turkish J Biochem. 41:S4 2016.

* First authorship shared

\section{PUBLICATIONS UNDER PROGRESS}

- Pradeep Shrestha, Ali Turan, Lalit Batra, Christine Akimana, Esma S. Yolcu, Haval Shirwan. Expression of FasL on donor grafts as a novel approach to prevention of acute graft-versus-host disease. (In preparation for submission to Blood)

- Wang, F.*, Shrestha, P.*, Woodward, K.B.*, Zhao, H., Williams, C., Bowen, W.S., Shea, L., Garcia, A.J., Hering , B., Yolcu, E.S, and Shirwan, H. Agonists of Fas and IL2 receptors work in synergy to induce robust tolerance to allografts. (manuscript in preparation)

* First authorship shared

\section{ABSTRACTS/PRESENTATIONS}

- Lalit Batra, Hampartsoum B. Barsoumian, Pradeep Shrestha, Jenci L. Hawthorne, William S. Bowen, Hong Zhao, Nejat K. Egilmez, Jorge G. Gomez-Gutierrez, Haval Shirwan, and Esma S. Yolcu. A novel agonist of CD137 immune checkpoint stimulator serves as a cancer immunoprevention agent with efficacy against various tumor types. American Association of Immunologists - Immunology 2019, San Diego, California, USA.

- Haval Shirwan, Hampartsoum B. Barsoumian, Lalit Batra, Pradeep Shrestha, William S. Bowen, Hong Zhao, Nejat K. Egilmez,Jorge G. Gomez-Gutierrez, Esma $\mathrm{S}$. Yolcu. A novel form of 4-1BB agonist shows robust immune protection against various tumor types through $\mathrm{CD}^{+}$memory-like $\mathrm{T}$ and $\mathrm{NK}$ cell axis. American Association for Cancer Research (AACR-2019) annual meeting, Atlanta, Georgia, USA. 
- Headen, D., Woodward, K., Weaver, J., Coronel, M.M., Shrestha, P., Bowen, W., Johnson, C., Shea, L., Yolcu, E., Garcia, A. and Shirwan, H., 2017, December. Allogeneic Graft Tolerance without Immunosuppression via Engineered Immunomodulatory Signal Presentation. In TISSUE ENGINEERING PART A (Vol. 23, pp. S41-S42). 140 HUGUENOT STREET, 3RD FL, NEW ROCHELLE, NY 10801 USA: MARY ANN LIEBERT, INC.

- Devon Headen, Jessica Weaver, Esma Yolcu, Kyle Woodward, Hong Zhao, Pradeep Shrestha, Haval Shirwan, Andrés J. García. Microgels presenting SAFasL achieve allogeneic islet graft acceptance without chronic immunosuppression. 2017 Society for Biomaterials Annual meetings. Minneapolis, Minnesota, April 58,2017

- Batra L, Shrestha P., Yolcu ES, Zhao H, Bowen WS, Woodward KB, Coronel MM, Tan M, Garcia AJ, Shirwan H. The transient display of a chimeric PD-L1 protein on pancreatic islets promotes indefinite survival in allogeneic recipients. 27th International Congress of the Transplantation Society, Madrid, Spain, June 30th to July 5th, 2018.

- Shrestha P, Bowen WS, Batra L, Tan M, Yolcu ES, Shirwan H. CD47 Overcomes Early Loss of Pancreatic Islet Grafts Transplanted Intraportally. $27^{\text {th }}$ International Congress of the Transplantation Society, Madrid, Spain, June $30^{\text {th }}$ to July $5^{\text {th }}, 2018$.

- Woodward KB, Headen DM, Shrestha P, Tan M, Bowen WS, Coronel MM, Hunckler MD, Weaver JD, Yolcu ES, Garcia AJ, Shirwan H. Immunomodulation with FA-FasL-engineered mucrogels achieves long-term survival of allogeneic islet grafts. $27^{\text {th }}$ International Congress of the Transplantation Society, Madrid, Spain, June $30^{\text {th }}$ to July $5^{\text {th }}, 2018$.

- Batra, L., P. Shrestha, E.S. Yolcu, H. Zhao, W.S. Bowen, K.B. Woodward, M. M. Coronel, M. Tan, A.J. García, and H. Shirwan. PD-L1-engineered pancreatic islet grafts overcome rejection in allogeneic recipients. J. Immunol. 194:176.6 (Abstr.)

- $\quad$ Shrestha, P., W.S. Bowen, L. Batra, M. Tan, E.S. Yolcu, and H. Shirwan. Display of CD47 protein on pancreatic islet grafts improves engraftment following intraportal transplantation. J. Immunol. 194:55.38 (Abstr.)

- Haval Shirwan, Kyle B. Woodward, Devon M. Headen, Hong Zhao, Pradeep Shrestha, Min Tan, William S. Bowen, María M. Coronel, Michael D. Hunckler, Jessica D. Weaver, Esma Yolcu, Andrés J. García. SA-FasL-engineered PEG microgels as a novel means of modulating immune response to allogeneic islet grafts. J. Immunol. 194:55.36 (Abstr.) 


\section{RESEARCH SKILLS and TECHNIQUES}

- In vivo animal procedures (murine model).

- Islet transplantation

- Bone marrow transplantation and graft versus host disease models

○ Microsurgical procedures

- Injections (subcutaneous, intravenous, intraperitoneal)

- Tissue harvest and analysis

- Flow cytometry (Proficiency in 14 parameters panel optimization and analysis)

- Humanized murine model

○ Human lymphocyte isolation and immunophenotyping

- Islet transplantation

- Acute GVHD model

- Molecular Techniques

○ PCR

O RT-qPCR

$\circ$ ELISA

- Histological analysis

- Tissue processing and sectioning

- Immunohistochemistry

$\circ$ Hematoxylin and Eosin staining

- Processing and analysis of tissues and organs

○ Tissue harvest and processing (Spleen, lymph nodes, liver, intestine)

- In vitro Cytokine, Mixed lymphocyte proliferation assays

- Softwares

- Microsoft office

- Graph pad prism

- Flowjo

$\circ$ GIMP \& Adobe photoshop 